\title{
Piecewise Linearization of Real Analytic Functions
}

\author{
By \\ Masahiro SHIotA*
}

\section{$\S 1$. Introduction}

Many mathematicians considered the problem of triangulations of algebraic sets, analytic sets, semi-algebraic sets, etc. [2], [4], [7], [8], [9]. [10], [14] and [19]. We want to consider more generally a global piecewise linearization of real analytic functions. For this, we need simultaneous triangulations of all levels of the functions.

A function on a polyhedron is called pieceuise linear $(=P L$, if we have a triangulation of the polyhedron such that the restriction of the function on each simplex is linear. A $C^{\infty}$ triangulation of a real analytic manifold.$M$ is a pair of a simplicial complex $K$ and a homeomorphism $g:|K| \rightarrow, I, K \mid$ meaning the underlying polyhedron of $K$, such that the restriction of $g$ on each simplex is a $C^{\infty}$ diffeomorphism onto the image. The existence of $C^{\infty}$ triangulation is wellknown (e.g. [12]). In this paper manifolds have not boundary unless otherwise specified.

Theorem I. Let $M \subset \boldsymbol{R}^{n},(K, g)$ be a real analytic manifolc of dimension $\div 4,5$ and its $C^{\infty}$ triangulation respectively, let $\varepsilon$ be a positive continuous function on $|K|$, and let $f$ be an analytic function on $M$. Then there exists a homeonorphism $=:|K| \rightarrow M$ such that $f \circ \tau$ is $P L$ and that $|\tau(x)-g(x)|<\varepsilon x ;$ ior $x \in|K|$.

Theorem II. Let $M$ be an analytic manifold of dimension 4 . and let $f$ be a proper analytic function on $M$. Then there exist a PL manifold $I^{\prime}$ and a homeomorphism $\tau: M M^{\prime} \rightarrow M$ such that $f \circ \tau$ is $P L$.

We call a set $X \subset \boldsymbol{R}^{n}$ subanalytic, (see [3]), if for any point $: \equiv \boldsymbol{R}^{n}$, there exist an open neighborhood $U$ of $x$ in $\boldsymbol{R}^{n}$ and a finite number of proper analytic maps $f_{i j}$ from real analytic manifolds to $U, j=1,2$, such that

$$
Y \cap U=\bigcup_{i}\left(\operatorname{Im} f_{\imath 1}-\operatorname{Im} f_{\imath 2}\right) .
$$

A continuous map $f: X_{1} \rightarrow X_{2}$ of subanalytic sets, $X_{1} \sqsubset \boldsymbol{R}^{n_{1}}, Y_{2}=R^{n_{2}}$, is called subanalytic if the graph is subanalytic in $\boldsymbol{R}^{n_{1}} \times \boldsymbol{R}^{n_{2}}$. We remark that a polyhedron $X$ closed in $R^{n}$ is subanalytic and that a $P L$ function on $X$ is subanalytic. We

Received June 21, 1983.

* Dept. of Math., College of General Education, Nagoya University, Máp: a «64, Japan. 
define the subanalyticness of a map or a subset of polyhedrons or of analytic manifolds after imbedding the spaces closedly in Euclidean spaces. It does not depend on the choice of imbeddings. Subanalytic versions of the above theorems are the following.

Theorem I'. Let $M \subset \boldsymbol{R}^{n}$ be a PL manifold of dimension $\neq 4,5$, let $s$ be a positive continuous function on $M$, and $f$ be a proper subanalytic function on $M$. Then there exists a homeomorphism $\tau$ of $M$ such that $f \circ \tau$ is $P L$ and that $\mid \tau(x)$ $x \mid<\varepsilon(x)$ for $x \equiv .1$.

Theorem II'. Let $M$ be a PL manifold of dimension 4, and $f$ be a proper subanalytic function on $M$. There exists a PL manifold $M^{\prime}$ and a homeonorphism $\tau: M^{\prime} \rightarrow M$ such that $f \circ \tau$ is $P L$.

As the existence of analytic triangulation follows from the $C^{\infty}$ triangulation (Proposition 6.11, these are generalizations of Theorems I, II in the case of proper $f$. The reason why we will treat the subanalytic case too is that it is convenient for the most part to consider our problem in the subanalytic category.

The process of the proof of Theorem $I$ is the following. At first we divide $M$ to semi-analytic $X_{1}, X_{2}$ so that $X_{1}$ contains all critical points of $f$ and that $f$ is locally constant on $X_{1}$. Secondly $f$ is piecewise linearized on a large domain of $X_{2}$, using Lemma 6.15 which is a consequence of the $C^{\infty}$ triangulation theory of $C^{\infty}$ manifolds. Thirdly we triangulate $X_{1}$ by Proposition 3.1, and then we piecewise linearize $f$ on a neighborhood of $X_{1}$ by Proposition 5.1. Lastly, applying Concordance Implies Isotopy Theorem of Kirby-Siebenmann [6], we piecewise linearize $f$ globally. Proposition 3.1 shows a fine triangulation of subanalytic sets. A triangulation of subanalytic sets is known [4]. But if we had only that triangulation, we might not apply Concordance Implies Isotopy Theorem, because the polyhedron treated might be not a $P L$ manifold. Proposition 5.1 is a subanalytic generalization of a result in [16] that if two analytic function germs vanish at the origin and have the same sign at each point near the origin, they are topologically equivalent (namely a local homeomorphism transfers one to the other).

The reason why we assume $f$ to be proper in Theorem II is that we can not apply Concordance Implies Isotopy Theorem but apply the Hauptrermutung theorem for 3-manifolds of Moise instead.

Two functions $f_{1}, f_{2}$ on a manifold $M$ is called topologically $R$ - $L$ equivalent if there exist homeomorphisms $\tau_{1}, \tau_{2}$ of $M, \boldsymbol{R}$ respectively such that $\tau_{2} \circ f_{1} \circ \tau_{1}=f_{2}$. An application of the theorems is the following.

Corollary III. Let $M$ be a real compact analytic (or compact $P L$ ) manifold of dimension $\neq 5$. Then the topological $R-L$ equivalence classes of all subanalytic functions on $M$ are countable. 
In $\$ 9$ we show necessary conditions for real (or complex; analytic maps to be piecewise linearizable. In this paper we use confusedly the differential of a function on $\boldsymbol{R}^{n}$ and the gradient by the usual Riemannian metric.

\section{$\S 2$. Subanalytic Sets}

Let us show some fundamental properties of subanalytic sets which we will need later. The results below without proof were proved in ${ }_{-3} 3$.

(2.1) The property of being subanalytic is closed under the following operations:

(2.1.1) finite union,

(2.1.2) finite intersection,

(2.1.3) difference of any two.

(2.2) Let $A \subset \boldsymbol{R}^{n}$ be a subanalytic set. Then the interior $-\hat{1}$ in $\boldsymbol{R}^{n}$, the closure $\bar{A}$ in $\boldsymbol{R}^{n}$ and every connected component of $A$ are subanalytic. Besides, $\operatorname{dim} A>$ $\operatorname{dim}(\bar{A}-A)$, and the family of all connected components of $A$ is locally finite.

(2.3) Any semi-analytic subset of $\boldsymbol{R}^{n}$ is subanalytic.

(2.4) Let $A \subset \boldsymbol{R}^{n}$ be closed, subanalytic and of dimension $r$. Then there exist an analytic manifold $M$ and a proper analytic map $f: M \rightarrow R^{n}$ such that $f(M)=$ -1. Moreover, for these $M$ and $f$, there exist an $r$-dimensional anaintic manifold $N$ and a proper analytic map $g: N \rightarrow M$ such that $f \circ g(N)=f i M$.

(2.5) Let $f: X \rightarrow I$ be a proper subanalytic map of analytic manifolds.

(2.5.1) If $B$ is a subanalytic subset of $Y^{r}$, then so is $f^{-1}(B)$ in $\mathrm{I}$.

(2.5.2) If $t$ is a subanalytic subset of $X$, then so is $f(A)$ in $I$.

(2.6) Let $A \subset \boldsymbol{R}^{n}, f: \boldsymbol{R}^{n} \rightarrow \boldsymbol{R}^{m}$ be subanalytic. Assume that the "estriction of $\int$ to $\bar{A}$ is proper (e.g. A is bounded). Then $f(A)$ is subanalstic.

(2.7) Let $A \subset \boldsymbol{R}^{n}$ be subanalytic. Then there exists a subanai. tic subset $A^{\prime}$ of At with $\operatorname{dim} A^{\prime}<\operatorname{dim} A$ such that $A-A^{\prime}$ is an analytic manifold.

Definition 2.8. Let $M, N \subset \boldsymbol{R}^{n}$ be $C^{\infty}$ manifolds. Assume that $M \cap N=\varnothing$. Let $y \in N^{r}$. We say that $(M, N)$ satisfies the Whitney condition at $y$ if the following is satisfied.

(2.8.1) If $\left\{x_{i}\right\},\left\{y_{l}\right\}$ are sequences in $M, N$ respectively, both converging to $y$, if the sequence of tangent spaces $\left\{T_{x_{i}} M\right\}$ converges to a subspace $T \subset \boldsymbol{R}^{n}$ (in the Grassmannian of $q$-dimensional subspaces of $\boldsymbol{R}^{n}$ where $q=\operatorname{dim} M$ ), and if the sequence $\left\{\overrightarrow{x_{i} y_{i}}\right\}$ of lines containing $x_{i}-y_{i}$ converges to a line $L \subset \boldsymbol{R}^{n}$ (in the Grassmannian of 1-dimensional subspaces of $\boldsymbol{R}^{n}$ ), then $L \subset T$. 
We say that $(M, N)$ satisfies the Whitney condition when it does so at every point in $N$.

Definition 2.9. A stratification of a subset $X$ of $\boldsymbol{R}^{n}$ is a partition $\left\{X_{l}\right\}$ of $X$ into bounded connected analytic submanifolds of $\boldsymbol{R}^{n}$ such that

(2.9.1) $\quad\left\{X_{2}\right\}$ is locally finite in $\boldsymbol{R}^{n}$, and that

(2.9.2) $\quad \bar{X}_{i} \Gamma_{1} I_{j}=\varnothing$ implies $\bar{X}_{i} \supset X_{\jmath}$.

If $\left\{X_{i}\right\}$ satisfies moreover

(2.9.3) for any $I_{\imath}, X_{\jmath},\left(X_{i}, X_{j}\right)$ satisfies the Whitney condition,

then we call it a Whitney stratification. The stratification is called subanalytic, if all strata are subanalytic. In Section 4 we weaken the above conditions on strata, we only assume they are $C^{\infty}$ manifolds and satisfy (2.9.1).

Definition 2.10. Let $\left\{A_{2}\right\},\left\{B_{u}\right\}$ be families of subsets of $\boldsymbol{R}^{n}$. We say that $\left\{A_{\nu}\right\}$ is compatible with $\left\{B_{l}\right\}$ if for each $\nu, \mu$.

$$
A_{2} \subset B_{l^{\prime}} \text { or } A_{2} r_{1} B_{l^{\prime \prime}}=\varnothing \text {. }
$$

(2.11) For a locally finite family $\left\{A_{2}\right\}$ of subanalytic sets in $\boldsymbol{R}^{n}$, there exists a Whitney sunanalytic stratification of $\boldsymbol{R}^{n}$ compatible with $\left\{A_{\nu}\right\}$.

In the case in which $\left\{A_{\nu}\right\}$ consists of one set, it is shown in [3]. The following is the key to the proof.

(2.12) Let $M, N \subset \boldsymbol{R}^{n}$ be subanalytic analytic manifolds with $M \cap N=\varnothing$. Then there exists a closed subanalytic subset $N^{\prime}$ of $N^{\prime}$ such that $\operatorname{dim} N^{\prime}<\operatorname{dim} N^{\prime}$ and that $\left(M, N-N^{\prime}\right)$ satisfies the Whitney condition.

Proof of (2.11). We may add $\boldsymbol{R}^{n}$ to $\left\{A_{2}\right\}$. We proceed by downward induction. Induction hypothesis: There exists a Whitney subanalytic stratification $\left\{X_{i}\right\}$ of a subanalytic subset $Y_{k+1}$ of $\boldsymbol{R}^{n}$ compatible with $\left\{A_{\nu}\right\}$ such that $Z_{k}=$ $R^{n}-Y_{k+1}$ is closed and of dimension $\leqq k$ and that each $X_{i}$ is of dimension $>k$ (the case $k=n$ is trivial). Put $\left\{A_{\nu}^{\prime}\right\}=\left\{A_{\nu} \cap Z_{k}\right\}$. For each $A_{\nu}^{\prime}$ we have a subanalytic partition $B_{\nu}$ and $C_{\nu}$ of $A_{\nu}^{\prime}$ by (2.7.12) such that $B_{\nu}$ is an analytic manifold of dimension $k$, that $C_{\nu}$ is of dimension $<k$ and that $\left(X_{i}, B_{\nu}\right)$ satisfies the Whitney condition for any $X_{i}$. Let $Z_{k-1}$ be the union of all $\bar{B}_{\nu}-B_{\nu}, \bar{C}_{\nu}$ and connected components of dimension $<k$ of the sets in the form $B_{\nu} \cap B_{\nu^{\prime}}$. Then $Z_{k-1}$ is closed, subanalytic and of dimension $<k$ by (2.1.2) and the locally finite assumption of $\left\{A_{2}\right\}$. It follows also that the union of $B_{\nu}-Z_{k-1}$ is $Z_{k}-Z_{k-1}$ because of $\boldsymbol{R}^{n} \in\left\{A_{2}\right\}$, and it is also a subanalytic analytic manifold. Let $\left\{X_{j}^{\prime}\right\}$ be all connected components of $Z_{k}-Z_{k-1}$. Then each $X_{j}^{\prime}$ is a subanalytic analytic manifold contained in some $B_{\nu}$, and moreover $X_{j}^{\prime} \cap B_{\nu^{\prime}} \neq \varnothing$ implies $B_{\nu^{\prime}} \supset X_{\jmath}^{\prime}$. Namely $\left\{X_{j}^{\prime}\right\}$ is compatible with $\left\{A_{2}\right\}$. Hence $\left\{X_{i}\right\} \cup\left\{X_{j}^{\prime}\right\}$ is a Whitney subana- 
lytic stratification of $Y_{k}=\boldsymbol{R}^{n}-Z_{k-1}$ compatible with $\left\{A_{2}\right\}$ whose strata are of dimension $\geqq k$. Therefore (2.11) follows.

(2.13) Let $X \subset \boldsymbol{R}^{m}$ be a closed subanalytic set.

(2.13.1) A map $f=\left(f_{1}, \cdots, f_{n}\right): X \rightarrow \boldsymbol{R}^{n}$ is subanalytic if and only if so are all $f_{i}$.

(2.13.2) The set of all subanalytic functions on $X$ is a ring.

(2.13.3) Let $f: X \rightarrow \boldsymbol{R}^{n}, g: \boldsymbol{R}^{n} \rightarrow \boldsymbol{R}^{l}$ be subanalytic. Then $g \circ f: X \rightarrow \boldsymbol{R}^{l}$ is subanalytic.

Proof. (2.13.1). Put

$$
Y=\operatorname{graph} f, Y_{\imath}=\operatorname{graph} f_{i}, i=1, \cdots, n \text {. }
$$

Let $\pi_{i}: \boldsymbol{R}^{m} \times \boldsymbol{R}^{n} \rightarrow \boldsymbol{R}^{m} \times \boldsymbol{R}, \tau_{i}: \boldsymbol{R}^{m} \times \boldsymbol{R}^{n} \rightarrow \boldsymbol{R}^{m} \times \boldsymbol{R}^{n}$ be defined by

$$
\begin{aligned}
& \pi_{i}\left(x_{1}, \cdots, x_{m}, y_{1}, \cdots, y_{n}\right)=\left(x_{1}, \cdots, x_{m}, y_{i}\right) \text { and } \\
& \tau_{i}\left(x_{1}, \cdots, x_{m}, y_{1}, \cdots, y_{n}\right)=\left(x_{1}, \cdots, x_{m}, y_{2}, \cdots, y_{i}, y_{1}, y_{2+1}, \cdots, y_{n}\right) .
\end{aligned}
$$

Then we have

$$
\begin{aligned}
& \pi_{i} Y^{\nu}=Y_{i}, i=1, \cdots, n, \\
& Y=\bigcap_{i=1}^{n} \tau_{\iota}\left(Y_{i} \times \boldsymbol{R}^{n-1}\right) .
\end{aligned}
$$

Since $X$ is closed in $\boldsymbol{R}^{m}$, so is $Y$ in $\boldsymbol{R}^{m} \times \boldsymbol{R}^{n}$. Hence, by (2.6) and the above former equality, if $Y$ is subanalytic. then so is $Y_{i}$. The converse follows from (2.1.2) and the latter equality.

(2.13.2). Let $f_{1}, f_{2}$ be subanalytic functions on $X$. Put

$$
\begin{aligned}
& Z_{-}=\left\{\left(y_{1}, y_{2}, y_{3}\right) \in \boldsymbol{R}^{3} \mid y_{3}=y_{1}-y_{2}\right\}, \\
& Z_{\times}=\left\{\left(y_{1}, y_{2}, y_{3}\right) \in \boldsymbol{R}^{3} \mid y_{3}=y_{1} y_{2}\right\}, \\
& Y_{i}=\operatorname{graph} f_{i}, i=1,2, \\
& Y_{-}=\operatorname{graph}\left(f_{1}-f_{2}\right), Y_{\lambda}=\operatorname{graph} \int_{1} f_{2} .
\end{aligned}
$$

Let $\pi: \boldsymbol{R}^{m} \times \boldsymbol{R}^{3} \rightarrow \boldsymbol{R}^{m} \times \boldsymbol{R}, \tau: \boldsymbol{R}^{m} \times \boldsymbol{R}^{3} \rightarrow \boldsymbol{R}^{m} \times \boldsymbol{R}^{3}$ be defined by

$$
\begin{aligned}
& \pi\left(x_{1}, \cdots, x_{m}, y_{1}, y_{2}, y_{3}\right)=\left(x_{1}, \cdots, x_{m}, y_{3}\right), \\
& \tau\left(x_{1}, \cdots, x_{m}, y_{1}, y_{2}^{\prime}, y_{3}\right)=\left(x_{1}, \cdots, x_{m}, y_{2}, y_{1}, y_{3}\right) .
\end{aligned}
$$

Then we have

$$
\begin{aligned}
& Y_{-}=\pi\left(\left(\boldsymbol{R}^{m} \times Z_{-}\right) \cap\left(Y_{1} \times \boldsymbol{R}^{2}\right) \cap \tau\left(Y_{2} \times \boldsymbol{R}^{2}\right)\right), \\
& Y_{>}=\pi\left(\left(\boldsymbol{R}^{m} \times Z_{\prime}\right) \cap\left(Y_{1} \times \boldsymbol{R}^{2}\right) \cap \tau\left(Y_{2} \times \boldsymbol{R}^{2}\right)\right) .
\end{aligned}
$$

Hence it follows from the closedness of $X,(2.1 .2)$ and (2.6) that $Y_{-}, Y_{\times}$are 
subanalytic.

As (2.13.3) follows in the same way as above, we omit its proof.

(2.14) Let $M \subset \boldsymbol{R}^{n}$ be a connected subanalytic analytic manifold of dimension $r$, and $f: \boldsymbol{R}^{n} \rightarrow \boldsymbol{R}^{m}$ be an analytic map. Then there exists a closed subanalytic set $A \subset M$ of dimension $<r$ such that the differential $d\left(\left.f\right|_{M}\right)$ of $\left.f\right|_{M}$ has constant rank on $M-A$.

Proof. Let $r^{\prime}$ be the maximal rank of $d\left(\left.f\right|_{x}\right)$. Since $M$ is connected, and since $f$ is analytic, the set

$$
Y=\left\{y \in M \mid \operatorname{rank} d\left(\left.f\right|_{M}\right)_{y}<r^{\prime}\right\}
$$

is an analytic subset of $M$ of dimension $<r$. Now by $(2.2), \operatorname{dim}(\bar{M}-M)<r$ and $\operatorname{dim} \bar{M}=r$. Hence there exist by (2.4) an analytic manifold $N$ of dimension $r$ and a proper analytic map $g: N \rightarrow \boldsymbol{R}^{n}$ such that $g(N)=\bar{M}$. Put

$$
\begin{aligned}
& X=\left\{x \in N \mid \operatorname{rank} d(f \circ g)_{x}<r^{\prime}\right\}, \\
& X^{\prime}=\left\{x \in N \mid \operatorname{rank} d g_{x}<r\right\} .
\end{aligned}
$$

Then $X, X^{\prime}$ are analytic subsets of $N$. Let $N_{1}$ be the union of all connected components of $N$ being contained in $X$, and let $N_{2}=N-N_{1}$. We see $N_{1} \subset X^{\prime}$ by reduction to absurdity. Assume $N_{1}-X^{\prime} \neq \varnothing$. Then $g\left(N_{1}-X^{\prime}\right)$ has inner points in $\bar{M}$. Hence it follows that $(M-Y) \cap g\left(N_{1}-X^{\prime}\right) \neq \varnothing$. This means that there exists a point $y \in N_{1}$ such that $g(y) \in M$, that $g$ is an imbedding on a neighborhood of $y$ and that rank $d\left(\left.f\right|_{M z}\right)_{g(y)}=r^{\prime}$, and hence rank $d(f \circ g)_{y}=r^{\prime}$. This contradicts the definitions of $X$ and $N_{1}$. Thus $N_{1} \subset X^{\prime}$. Hence we have $\operatorname{dim} g\left(N_{1}\right)$ $<r$, which together with the inequality $\operatorname{dim}\left(N_{2} \cap X\right)<r$ implies $\operatorname{dim} g(X)<r$. Put $A=M \cap \overline{g(X)}$, then the lemma follows from (2.2.6).

(2.15) Let $f: \boldsymbol{R}^{n} \rightarrow \boldsymbol{R}^{m}$ be analytic, and $X, X_{i}, i=1,2, \cdots$ be locally finite subanalytic sets in $\boldsymbol{R}^{n}$ with $X \supset X_{i}$. Then there exists a Whitney subanalytic stratification $\left\{Y_{\imath}\right\}$ of $X$ compatible with $\left\{X_{i}\right\}$ such that $\left.f\right|_{Y_{i}}$ for each $i$ has constant rank.

Proof. We prove it by induction on $r=\operatorname{dim} X$. The case $r=0$ is trivial. Hence we assume it for $\operatorname{dim} X<r$. By (2.11) there exists a subanalytic stratification $\left\{X_{i=1}^{\prime}\right\}_{i=1} \ldots$ of $X$ compatible with $\left\{X_{i}\right\}$. Let $\operatorname{dim} X_{i}^{\prime}=r$ for $i \in \Lambda_{1}, \Lambda_{1} \subset N$, and $\operatorname{dim} X_{i}^{\prime}<r$ for $i \in \Lambda_{1}$. Apply (2.14) to each $X_{i}^{\prime}, i \in \Lambda_{1}$. Then we have a subanalytic set $Z_{i}$ in $X_{i}^{\prime}$ of dimension $<r$ such that $\left.f\right|_{x_{i}^{\prime}-Z_{i}}$ has constant rank. Apply once more (2.11) to $\left\{X_{i}^{\prime}-Z_{i}, Z_{i}, X_{j}^{\prime} \mid i \in \Lambda_{1}, j \oplus \Lambda_{1}\right\}$, and let $\left\{Y_{i=1}^{\prime}\right\}_{i=1}$ be the resulting Whitney subanalytic stratification of $X$ compatible with $\left\{X_{i}^{\prime}-Z_{i}\right.$, $\left.Z_{i}, X_{j}^{\prime} \mid i \in \Lambda_{1}, j \notin \Lambda_{1}\right\}$ with $\operatorname{dim} Y_{i}^{\prime}=r$ for $i \in \Lambda_{2}, \Lambda_{2} \subset N$, and $\operatorname{dim} Y_{i}^{\prime}<r$ for $i \notin \Lambda_{2}$. It clearly follows that $\left\{Y_{i}^{\prime}\right\}$ is compatible with $\left\{X_{i}\right\}$. By induction hypothesis there exists a Whitney subanalytic stratification $\left\{Y_{\jmath}^{\prime \prime}\right\}$ of $\bigcup_{\imath \notin \Lambda_{2}} Y_{i}^{\prime}$ compatible with 
$\left\{Y_{i}^{\prime}\right\}_{i \notin \perp_{2}}$ such that $\left.f\right|_{r_{j}^{\prime \prime}} ^{\prime \prime}$ for each $j$ has constant rank. Then $\left(Y_{i}^{\prime}, Y_{j}^{\prime \prime}\right)$ for any $i \in \Lambda_{2}$ and any $j$ satisfies the Whitney condition, because $Y_{j}^{\prime \prime}$ is contained in some $Y_{i^{\prime}}^{\prime}, i^{\prime} \notin \Lambda_{2}$, and $\left(Y_{i}^{\prime}, Y_{i^{\prime}}^{\prime}\right)$ satisfies the Whitney condition. Hence $\left\{Y_{i}^{\prime}\right\}_{i \notin .1_{2}} \cup\left\{Y_{j}^{\prime \prime}\right\}$ satisfies the required properties.

\section{$\S 3$. Triangulation of Subanalytic Sets}

The purpose of this section is to prove the following refinement of a result in $[4]$.

Proposition 3.1. Let $K$ be a triangulation of $\boldsymbol{R}^{n}$. Let $\left\{A_{i}\right\}$ be a locally finite family of subanalytic subsets of $\boldsymbol{R}^{n}$. Then there exist a subdivision $K^{\prime \prime}$ of $K$ and a homeomorphism $\tau$ of $\boldsymbol{R}^{n}$ such that

(3.1.1) $\tau$ is subanalytic,

(3.1.2) $\tau(\sigma)=\sigma$ for any $\sigma \in K$,

(3.1.3) for any $\sigma \in K^{\prime \prime}, \tau(\dot{\sigma})$ is an analytic submanifold of $\boldsymbol{R}^{n}$ and $\left.\tau\right|_{\dot{\sigma}}: \dot{\sigma} \rightarrow \tau(\dot{\sigma})$ is an analytic diffeomorphism and that

(3.1.4) $\left\{\tau(\stackrel{\circ}{\sigma}) \mid \sigma \in K^{\prime}\right\}$ is compatible with $\left\{A_{i}\right\}$.

A simplex (or a cell) is understood to be a closed one, and $\sigma$ is the interior of a simplex or a cell $\sigma$. By $\partial \sigma$ we denote the boundary. In this paper we always consider the usual polyhedron structure on $\boldsymbol{R}^{n}$.

The condition (3.1.2) was not under consideration in [4], and it will play an important part in our application (see Remark 6.23). We proceed with the proof in a similar way to [10].

Let $A \subset \boldsymbol{R}^{n}$ be subanalytic, and $c \in \boldsymbol{R}^{n}$ be a point. A line $\lambda$ through $c$ is called non-singular for $A$ at $c$ if $\lambda \cap A=c$ or $=\varnothing$ in a neighborhood of $c$. In the other case, namely when $\operatorname{dim}(\lambda \cap A)=1$ in any neighborhood of $c, \lambda$ is called singular for $A$ at $c$. If $\lambda$ is non-singular for $A$ at any point of $\lambda$, we call $\lambda$ nonsingular for $A$. In the other case $\lambda$ is called singular for $A$.

Let $a \in \boldsymbol{R}^{n}$ be a point, and $S_{a}^{n-1} \subset \boldsymbol{R}^{n}$ denote the sphere centered at $a$ with radius 1. Let $q_{a}: \boldsymbol{R}^{n}-a \rightarrow S_{a}^{n-1}, q_{a}^{\prime}: \boldsymbol{R}^{n}-a \rightarrow S_{a}^{n-1} \times \boldsymbol{R}$ be defined by

$$
\begin{aligned}
& q_{a}(x)=a+(x-a) /|x-a|, \\
& q_{a}^{\prime}(x)=\left(q_{a}(x),|x-a|\right) .
\end{aligned}
$$

Let $X_{1}, X_{2} \subset \boldsymbol{R}^{n}$ and I $\subset S_{a}^{n-1}$ be subsets with $a \notin X_{1}, X_{2}$. Assume the existence of functions $\phi_{1}, \phi_{2}$ on $Y$ such that $q_{a}^{\prime}\left(X_{2}\right)=\operatorname{graph} \phi_{\imath}, i=1,2$. Then we write

$$
\begin{array}{ll}
X_{1}<X_{2} \text { with respect to }\left(\boldsymbol{R}^{n}, a\right) & \text { if } \phi_{1}<\dot{\psi}_{2}, \\
X_{1} \leqq X_{2} \text { with respect to }\left(\boldsymbol{R}^{n}, a\right) & \text { if } \varphi_{1} \leqq \psi_{2} .
\end{array}
$$


In either case we denote by $\left(X_{1}, X_{2}\right)$ and $\left[X_{1}, X_{2}\right]$ the sets

$$
\begin{aligned}
& \left\{q_{a}^{\prime-1}(y, t)_{i}^{\prime}(y, t) \in Y \times \boldsymbol{R}, \phi_{1}(y)<t<\phi_{2}(y)\right\} \quad \text { and } \\
& \left\{q_{a}^{\prime-1}(y, t) \mid \phi_{1}(y) \leqq t \leqq \phi_{2}(y)\right\}
\end{aligned}
$$

respectively.

An analytic submanifold $\Gamma \subset \boldsymbol{R}^{n}$ is called topographic with respect to $\left(\boldsymbol{R}^{n}, a\right)$ if $a \notin \Gamma$, if $q_{a}(\Gamma)$ is an analytic manifold and if $\left.q_{a}\right|_{\Gamma}: \Gamma \rightarrow q_{a}(\Gamma)$ is an analytic diffeomorphism. Let $p: \boldsymbol{R}^{n} \times \boldsymbol{R}^{m} \rightarrow \boldsymbol{R}^{n}$ be the projection. An analytic submanifold $\Gamma \subset \boldsymbol{R}^{n} \times \boldsymbol{R}^{m}$ is called topographic with respect to $\boldsymbol{R}^{m}$ if $p(\Gamma)$ is an analytic manifold and if $\left.p\right|_{\Gamma}: \Gamma \rightarrow p(\Gamma)$ is an analytic diffeomorphism. We remark that an analytic submanifold $\Gamma \subset \boldsymbol{R}^{n}$ is topographic with respect to $\left(\boldsymbol{R}^{n}, a\right)$ if and only if $a \notin \Gamma$ and if $q_{a}^{\prime}(\Gamma) \subset \boldsymbol{R}^{n} \times \boldsymbol{R}$ is topographic with respect to $\boldsymbol{R}$.

We say that a subset $X \subset \boldsymbol{R}^{n}-a$ has property $(P)$ with respect to $\left(\boldsymbol{R}^{n}, a\right)$ if the restriction $\left.q_{a}\right|_{X}$ is an open map. If $X \subset \boldsymbol{R}^{n} \times \boldsymbol{R}^{m}$, we define the property $(P)$ of $X$ with respect to $\boldsymbol{R}^{m}$ in the same way as above. We also remark that $X$ $\subset \boldsymbol{R}^{n}$ has property $(P)$ with respect to $\left(\boldsymbol{R}^{n}, a\right)$ if and only if $a \oplus \Gamma$ and if $q_{a}^{\prime}(X)$ $\subset \boldsymbol{R}^{n} \times \boldsymbol{R}$ has property $(P)$ with respect to $\boldsymbol{R}$.

Lemma 3.2. Let $A \subset \boldsymbol{R}^{n}$ be subanalytic and of dimension $<n$. Then the union of all points on lines singular for $A$ is meager in $\boldsymbol{R}^{n}$ (a countable union of nowhere dense sets).

Proof (Compare with Lemma 3 in $[10]$ ). Let $\left\{4_{l}\right\}$ be a subanalytic stratification of $A$ (2.11). Since $\left\{A_{i}\right\}$ is locally finite, any singular line for $A$ is singular for some $A_{i}$. Hence we can assume $A$ to be an analytic submanifold of $\boldsymbol{R}^{n}$. Then there exist an open set $U \subset \boldsymbol{R}^{n}$ and an analytic function $f$ on $U$ such that $A \subset U, A=f^{-1}(0)$. Put

$$
\begin{aligned}
& \Theta=\left\{(x, y) \in U \times \boldsymbol{R}^{n} \mid y \neq 0, \text { the line through } x\right. \\
& \text { and } x+y \text { is singular for } A \text { at } x\}, \\
& \pi(x, y)=x+y \quad \text { for } \quad(x, y) \in U \times \boldsymbol{R}^{n} .
\end{aligned}
$$

Then $\Theta$ is a semi-analytic subset of $U \times \boldsymbol{R}^{n}[7]$, because of

$$
\Theta=\left\{(x, y) \in U \times \boldsymbol{R}^{n} \mid y \neq 0, \frac{\partial^{i}}{\partial t^{i}} f(x+t y)_{t=0}=0, i=1,2, \cdots\right\} .
$$

If a line $\lambda$ is singular for $A$ at a point $x, f$ vanishes identically on the connected component of $\lambda \cap U$ containing $x$. This implies that for any point $x \in U$, there exist neighborhoods $V, V^{\prime}$ of $x, 0$ respectively such that $\pi\left(\Theta \cap\left(V \times V^{\prime}\right)\right)$ is contained in $f^{-1}(0)$. Hence $\pi\left(\Theta \cap\left(V \times V^{\prime}\right)\right)$ is of dimension $<n$. On the other hand we have

$$
\Theta \cap\left(V \times \boldsymbol{R}^{n}\right)=\left\{\left(x^{\prime}, t y\right) \mid\left(x^{\prime}, y\right) \in \Theta \cap\left(V \times V^{\prime}\right), t \neq 0 \in \boldsymbol{R}\right\} .
$$


Choose as $V$ a closed small ball centered at $x$. Then $\Theta \cap\left(V \times \boldsymbol{R}^{n}\right)$ is semi-analytic in $\boldsymbol{R}^{n} \times \boldsymbol{R}^{n}$ and hence subanalytic by (2.3). Apply (2.11) to $\Theta \cap\left(V \times S_{0}^{n-1}\right)$, and choose a finite stratification of the set. Then, by (3.2.1) there exists a finite stratification $\left\{M_{i}\right\}$ of $\Theta \cap\left(V \times \boldsymbol{R}^{n}\right)$ such that for each $i$, if $\left(x^{\prime}, y\right) \in M_{i}, t>0$, then $\left(x^{\prime}, t y\right) \in M_{i}$. Therefore the rank of the restriction $\left.\pi\right|_{x_{i}}$ for each $i$ is smaller than $n$ in a neighborhood of $(x, 0)$ and hence on $M_{\iota}$. This implies that $\pi(\Theta)$ is meager in $\boldsymbol{R}^{n}$. As the set in question is contained in $\pi(\Theta) \cup A$, Lemma 3.2 follows.

Lemma 3.2'. Let $A$ be the same as Lemma 3.2. Let $B$ be the subset of the projective space $P^{n-1}(\boldsymbol{R})$ consisting of lines $L$ such that $a+L=\{a+x \mid x \in L\}$ are singular for $A$ at a for some $a \in A$. Then $B$ is meager in $P^{n-1}(\boldsymbol{R})$.

Proof. We reduce in the same way as the proof of Lemma 3.2 the problem to the case in which $A$ is an analytic submanifold of $\boldsymbol{R}^{n}$ and hence in which $A$ is the zero set of an analytic function on an open set. Then the lemma is a special case of Lemma 3, [10].

Lemma 3.3. Let $A_{2} \subset \boldsymbol{R}^{n}, i=1,2, \cdots$ be subanalytic sets of dimension $<n$, and $a \equiv \boldsymbol{R}^{n}$ be a point. Then there exist a point $b \in \boldsymbol{R}^{n}$ arbitrarily close to a such that any line through $b$ is non-singular simultaneously for all $A_{2}$.

Proof. Trivial from Lemma 3.2.

Lemma 3.3'. Let $A_{2}$ be the same as Lemma 3.3, and let $L \subseteq P^{n-1}(\boldsymbol{R})$. Then there exists $L^{\prime} \in P^{n-1}(\boldsymbol{R})$ arbitrarily close to $L$ such that for any $a \in \boldsymbol{R}^{n}, a+L^{\prime}$ is non-singular simultaneously for all $A_{i}$.

Proof. It follows from Lemma 3.2'.

Lemma 3.4. Let $\left\{A_{\iota}\right\}$ be a finite family of compact subanalytic sets in $\boldsymbol{R}^{n}$ $\therefore \boldsymbol{R}$. Assume that for any $x \in \boldsymbol{R}^{n}$, the line $x \times \boldsymbol{R}$ is non-singular for $\cup \mathcal{A}_{i}$. Then there exists a subanalytic stratification $\left\{X_{2}\right\}$ of $\cup_{-} A_{i}$ compatible with $\left\{A_{2}\right\}$ such that each $X_{i}$ is topographic with respect to $\boldsymbol{R}$.

Proof. Let $r$ be the dimension of $A=\cup H_{1}$. We prove the lemma by induction on $r$. The case $r=0$ is trivial. Assume the lemma for dimension $<r$. Let $p: \boldsymbol{R}^{n} \times \boldsymbol{R} \rightarrow \boldsymbol{R}^{n}$ be the projection. By the non-singularity assumption on $A_{i}$ we have $\operatorname{dim} p(A)=r$. Apply $(2.7)$ to $\{p(A)\}$ and (2.11) to $\left\{A_{i}\right\}$. Then there exist a subanalytic stratification $\left\{B_{i}\right\}_{i=1, \ldots, s}$ of $A$ compatible with $\left\{A_{i}\right\}$ and a subanalytic partition $C_{1}, C_{2}$ of $p(A)$ such that the dimensions of $B_{\imath}$ and $C_{j}$ are equal to $r$ for $i=1, \cdots, s^{\prime}, j=1$ and smaller than $r$ for $i=s^{\prime}+1, \cdots, s, j=2$ and that $C_{1}$ is an analytic manifold. Now apply (2.14) to $p$ and each $B_{i}, i=1, \cdots, s^{\prime}$. Then we have subanalytic subsets $D_{i} \subset B_{i}$ of dimension $<r$ closed in $B_{1}$ such that $d\left(\left.p\right|_{B_{l}}\right)$ has rank $r$ on $B_{i}-D_{i}$ by the non-singularity assumption.

Put 


$$
\begin{aligned}
& M=C_{1}-p\left(\bigcup_{i=1}^{s^{\prime}} D_{i} \cup \bigcup_{i=s^{\prime}+1}^{s} B_{i}\right) \\
& N=p^{-1}(M) \cap A .
\end{aligned}
$$

Then (i) $M, N$ are $r$-dimensional subanalytic analytic manifolds by (2.5), (ii) $p(N)=M$, (iii) $\left.p\right|_{N}$ is an immersion and (iv) $\operatorname{dim}(A-N)=\operatorname{dim}(p(A)-M)<r$. Let $\left\{X_{i}\right\}_{i=1, \ldots, k}$ be the connected components of $N$. Then each $p\left(X_{i}\right)$ is an analytic manifold, moreover it follows from the compactness of $A$ that $\left(X_{i},\left.p\right|_{x_{i}}, p\left(X_{i}\right)\right)$ is an analytic covering. The covering is 1 -fold. Indeed, if not so, we have a subset $X_{i}^{\prime} \subset X_{i}$ homeomorphic to $S^{\prime}$ such that $\left(X_{i}^{\prime},\left.p\right|_{x_{i}^{\prime}}, p\left(X_{i}^{\prime}\right)\right)$ is a non-onefold covering. We easily see that to be impossible by the inclusion $X_{i}^{\prime} \subset p\left(X_{\imath}^{\prime}\right) \times \boldsymbol{R}$. Thus $X_{i}$ are topographic with respect to $\boldsymbol{R}$. We remark that $\left\{X_{i}\right\}$ is compatible with $\left\{B_{i}\right\}$ and hence with $\left\{A_{i}\right\}$.

If a connected subset $Y$ of $A-N$ is compatible with $\left\{A_{i}^{\prime}\right\}=\left\{\bar{D}_{i}, \bar{B}_{j} \mid i=1, \cdots\right.$, $\left.s^{\prime}, j=s^{\prime}+1, \cdots, s\right\}$, then we see easily $Y \subset B_{j}$ for some $j$, hence $Y$ is compatible with $\left\{A_{i}\right\}$. Hence for the proof we only need a subanalytic stratification $\left\{X_{i}\right\}_{i=k+1, \cdots, k^{\prime}}$ of $A-N$ compatible with $\left\{A_{i}^{\prime}\right\} \cup\{A-N\}$ such that each $X_{i}$ is topographic with respect to $\boldsymbol{R}$. Since $A-N$ is compact and of dimension $<r$, and since $A_{i}^{\prime} \subset A-N$, it follows from the induction hypothesis, hence the lemma is proved.

Lemma 3.5. Let $A \subset \boldsymbol{R}^{n} \times \boldsymbol{R}$ be a compact subanalytic set. Assume that $0 \in A$ and that for any $x \in \boldsymbol{R}^{n}$, the line $x \times \boldsymbol{R}$ is non-singular for $A$. Then there exists a compact subanalytic set $B \subset \boldsymbol{R}^{n} \times \boldsymbol{R}$ such that $A \subset B$, that $x \times \boldsymbol{R}$ for any $x \in \boldsymbol{R}^{n}$ is non-singular for $B$ and that $B \cap U$ has property $(P)$ with respect to $\boldsymbol{R}$ for some open subset $U$ of $\boldsymbol{R}^{n} \times \boldsymbol{R}$ containing 0 .

Proof. We prove the lemma by induction on $n$. If $n=0$, the lemma is trivial. Hence we assume it for $n-1$. Let $p: \boldsymbol{R}^{n} \times \boldsymbol{R} \rightarrow \boldsymbol{R}^{n}$ be the projection. By Lemma 3.4, there exists a subanalytic partition $A_{1}, A_{2}$ of $A$ such that $A_{1}$ (may be empty) is an analytic manifold of dimension $n$, that $A_{2}$ is compact and of dimension $<n$ and that $\left.\phi\right|_{A_{1}}$ is open. We clearly have $A_{2} \neq \varnothing$. We assume moreover $0 \in A_{2}$, otherwise we have nothing to do. Since $\operatorname{dim} p\left(A_{2}\right)<n$, there exists by Lemma $3.3^{\prime}$ a line $L \in P^{n-1}(\boldsymbol{R})$ such that for any $a \in \boldsymbol{R}^{n}, a+L$ is nonsingular for $p\left(A_{2}\right)$. Hence, without loss of generality we can assume that for any $x^{\prime} \in \boldsymbol{R}^{n-1}$ the line $x^{\prime} \times \boldsymbol{R} \subset \boldsymbol{R}^{n-1} \times \boldsymbol{R}=\boldsymbol{R}^{n}$ is non-singular for $p\left(A_{2}\right)$. This means that for any $x^{\prime} \in \boldsymbol{R}^{n-1}, x^{\prime} \times \boldsymbol{R} \times \boldsymbol{R} \cap A_{2}$ is of dimension 0 and hence consists of finite points.

Let $p_{2}: \boldsymbol{R}^{n} \times \boldsymbol{R} \rightarrow \boldsymbol{R}^{n-1}$ 冫 $\boldsymbol{R}$ be the projection defined by

$$
p_{2}\left(x_{1}, \cdots, x_{n}, y\right)=\left(x_{1}, \cdots, x_{n-1}, y\right) .
$$

Then it follows that $p_{2}\left(A_{2}\right)$ is a compact subanalytic set containing 0 and that for any $x^{\prime} \in \boldsymbol{R}^{n-1}$, the line $x^{\prime} \times \boldsymbol{R}$ is non-singular for $p_{2}\left(A_{2}\right)$. So by induction 
hypothesis we have a compact subanalytic set $B_{2} \subset \boldsymbol{R}^{n-1} \times \boldsymbol{R}$ such that $p_{2}\left(A_{2}\right) \subset B_{2}$, that $x^{\prime} \times \boldsymbol{R}$ for any $x^{\prime} \in \boldsymbol{R}^{n-1}$ is non-singular for $B_{2}$ and that $B$ has property $(P)$ with respect to $\boldsymbol{R}$ in a neighborhood of 0 . Hence $B^{\prime}=p_{2}^{-1}\left(B_{2}\right) \cap$ (a large closed ball centered at 0 ) is a compact subanalytic set in $\boldsymbol{R}^{n} \times \boldsymbol{R}$ such that $A_{2} \subset B^{\prime}$, that $x, \boldsymbol{R}$ for any $x \in \boldsymbol{R}^{n}$ is non-singular for $B^{\prime}$ and that $B^{\prime}$ has property $(P)$ with respect to $\boldsymbol{R}$ in a neighborhood of 0 . Therefore $B=A_{1} \cup B^{\prime}=A \cup B^{\prime}$ satisfies the required properties in Lemma 3.5 .

Using $q_{a}^{\prime}$, we immediately obtain from Lemmas $3.4,5$ the following.

Lemma 3.4'. Let $\left\{A_{2}\right\}$ be a finite family of compact subanalytic subsets of $\boldsymbol{R}^{n}$, and $a \in \boldsymbol{R}^{n}$ be a point outside of $A=\cup A_{l}$. Assume that any line through $a$ is non-singular for $A$. Then there exists a subanalytic stratification $\left\{X_{j}\right\}$ of $A$ compatible with $\left\{A_{\imath}\right\}$ such that each $X_{j}$ is topographic with respect to $\left(\boldsymbol{R}^{n}, a\right)$.

Lemma 3.5'. Let $A \subset \boldsymbol{R}^{n}$ be a compact subanalytic set, and let $a \subseteq \boldsymbol{R}^{n}-A$, $b \equiv A$. Assume that any line through $a$ is non-singular for $A$. Then there exists a compact subanalytic set $B \subset \boldsymbol{R}^{n}$ such that $A \subset B$ and $a \notin B$, that any line through $a$ is non-singular for $B$ and that $B \cap L^{T}$ has property $(P)$ with respect to $\left(R^{n}\right.$, a) for some open neighborhood $U$ of $b$.

Lemma 3.6. Under the same assumption as Lemma 3.5', there exist a compact subanalytic set $B \subset \boldsymbol{R}^{n}$ and a subanalytic stratification $\left\{B_{i}\right\}$ of $B$ such that $A \cap U$ is a union of some $B_{i}$ 's for a neighborhood $U$ of $b$, that each $B_{i}$ is topographic with respect to $\left(\boldsymbol{R}^{n}\right.$, a) and that $B$ has property $(P)$ with respect to $\left(\boldsymbol{R}^{n}, a\right)$.

Proof. Assume $a=(0, \cdots, 0), b=(0, \cdots, 0,1)$. Let $\delta_{n}, \delta>0$ be small numbers. Put

$$
\begin{aligned}
& h(x)=\left(x_{1}^{2}+\cdots+x_{n-1}^{2}\right)^{1,2}-\partial_{n} x_{n}, \\
& Q=\left\{x \in \boldsymbol{R}^{n}|h(x) \leqq 0,| 1-|x| \mid \leqq \hat{o}\right\} .
\end{aligned}
$$

Then $Q$ is a small neighborhood of $b$. It is easy to find an analytic diffeomorphism $\pi$ of $\mathbb{R}^{n}$-0 such that

$$
\begin{aligned}
& |\pi(x)|=|x|, \\
& \pi(x)=x \quad \text { if } \quad h(x)=0, \\
& h(\pi(x))>0 \quad \text { if } \quad h(x)<0, \\
& h(\pi(x))<0 \quad \text { if } \quad h(x)>0,
\end{aligned}
$$

and that $\pi$ carries each half-line with end point 0 to some such half-line.

Apply Lemma $3.5^{\prime}$ to $A$, let $A_{1}$ be the intersection of $Q$ and the resulting subanalytic set, and put 


$$
\begin{aligned}
& A_{2}=A_{1} \cap \partial Q, \quad A_{3}=\pi^{\prime}\left(A_{1}\right), \\
& A_{4}=A \cap Q, \quad B=A_{1} \cup A_{3} .
\end{aligned}
$$

Then $A_{4} \subset B$, and any line through 0 is non-singular for $A_{1}, A_{3}$ and hence for $B$. Choose $\delta_{n}, \delta$ small enough. Then $B-A_{2}$ has property $(P)$ with respect to $\left(\boldsymbol{R}^{n}, 0\right)$, and we have $0 \times \boldsymbol{R} \cap A_{1}=b$. Furthermore, we lessenning $\delta_{n}$ so that

$$
A_{2} \subset\left\{x \in \boldsymbol{R}^{n} \mid h(x)=0\right\},
$$

$B$ comes to have property $(P)$ with respect to $\left(\boldsymbol{R}^{n}, 0\right)$. Now apply Lemma $3.4^{\prime}$ to $\left\{A_{i}\right\}_{i=1, \ldots, 4}$. Then the resulting subanalytic stratification $\left\{B_{i}\right\}$ of $B$ satisfies the required properties.

Let $\sigma \subset \boldsymbol{R}^{n}$ be an $n$-simplex, and $a \in \delta$. We define $p_{\sigma a}: \boldsymbol{R}^{n}-a \rightarrow \partial \sigma$ by $p_{\sigma a}(x)=$ the intersection of $\partial \sigma$ and the half-line through $x$ with end point $a$.

For cell complexes $K_{1}, K_{2}, K_{1} \times K_{2}$ denotes the product cell complex $\left\{\sigma_{1} \times\right.$ $\left.\sigma_{2} \mid \sigma_{1} \in K_{1}, \delta_{2} \in K_{2}\right\}$. For a point $a$, a simplex $\sigma$ and a complex $K$, we write as $a * \sigma$ the cone with vertex $a$ and base $\sigma$, and as $a * K$ the complex generated by $a * \sigma^{\prime}, \sigma^{\prime} \in K$, if they are well-defined.

Let $\sigma$ be a simplex, and $a \in \dot{\sigma}$. For any homeomorphism $\phi$ of $\partial \sigma$, we define the conic extension homeomorphism $a * \psi$ of $\sigma$ by

where

$$
a * \phi(x)= \begin{cases}t\left(\psi^{\circ} p_{\sigma a}(x)-a\right)+a & \text { for } x \neq a \\ x & \text { for } x=a\end{cases}
$$

Then we have

$$
t\left(p_{\sigma a}(x)-a\right)=x-a .
$$

Lemma 3.7. If $\phi$ is subanalytic, so is $a * \psi$.

Proof. Let $\boldsymbol{R}^{n}$ be the affine space spanned by $\sigma$. Assume $a=0$. By (2.4) there exist a compact analytic manifold $M$ and an analytic map $f: M \rightarrow \boldsymbol{R}^{n} \times \boldsymbol{R}^{n}$ such that $f(M)=\operatorname{graph} \psi$. Let $F: M \times[0,1] \rightarrow \boldsymbol{R}^{n} \times \boldsymbol{R}^{n}$ be defined by $F(x, t)=$ $t f(x)$. Then $F(M \times[0,1])=\operatorname{graph}(0 * \psi)$, and it is a subanalytic set. Clearly $0 * \psi$ is continuous. Hence $a * \phi$ is subanalytic.

Proposition 3.1'. Let $K$ be a simplicial complex in $\boldsymbol{R}^{n}$. Assume $|K|$ to be closed in $\boldsymbol{R}^{n}$. Let $\left\{A_{i}\right\}$ be a locally finite family of subanalytic subsets of $\boldsymbol{R}^{n}$ contained in $|K|$. Then there exist a subdivision $K^{\prime}$ of $K$ and a homeomorphism $\tau$ of $|K|$ such that the conditions (3.1.1), .., (3.1.4) in Proposition 3.1 are satisfied.

Proof of Proposition 3.1. Trivial if we assume Proposition 3.1'.

Proof of Proposition 3.1'. Let $m$ be the dimension of $K$. We will prove the proposition by induction on $m$. The case $m=0$ is trivial. Assume the proposition for dimension $\leqq m-1$. Since a simplex is a subanalytic set, we assume $\left\{A_{l}\right\} \supset K^{m-1}$, where $K^{m-1}$ is the $(m-1)$-skeleton of $K$. Considering $A_{i} \cap \sigma$ for 
each $A_{i}$ and $\sigma \in K$, we may suppose that each $A_{i}$ is contained in some $\sigma \in K$.

Moreover we can assume that $A_{\imath}$ are closed. Indeed, if $A_{i}$ is not closed, we replace $A_{i}$ by a finite system of closed subanalytic sets as follows. Let $A_{i 0}=A_{i}$, and $A_{i \jmath+1}=\bar{A}_{i \jmath}-A_{i \jmath}, j=0,1, \cdots$. Then, by (2.2), $\operatorname{dim} A_{i j+1}<\operatorname{dim} A_{\imath \jmath}$ so long as $A_{i j} \neq \varnothing$. Replace $A_{\iota}$ by $\left\{\bar{A}_{i j}\right\}$.

We may assume also $\operatorname{dim} A_{i}<m$ for each $i$. In fact, if $\operatorname{dim} A_{i}=m$ and $A_{i}$ is contained in $\sigma \in K$, then we consider $A_{i}-\operatorname{Int}\left(A_{i}\right)$ in place of $A_{i}$. Here $\operatorname{Int}\left(A_{\imath}\right)$ means the interior of $A_{i}$ in the affine space spanned by $\sigma$. Then a partition of $|K|$ compatible with the new family is automatically compatible with $\left\{A_{\imath}\right\}$.

(I) Let $\sigma$ be an $m$-simplex of $K$, and $\Sigma$ denote the affine space spanned by $\sigma$. We regard $\Sigma$ as a Euclidean space too. Then there exist a point $a_{\sigma}$ of $\sigma$ and a finite family $\mathcal{C}_{\sigma}$ of subanalytic analytic submanifolds of $\Sigma$ such that

(I.1) each $\Gamma \in \mathcal{C}_{\sigma}$ is contained in $\sigma$, topographic with respect to $\left(\Sigma, a_{\sigma}\right)$,

(I.2) the union $S_{\sigma}$ of all $\Gamma \in C^{3}{ }_{\sigma}$ is closed and has property $(P)$ with respect to $\left(\Sigma, a_{\sigma}\right)$, and that

(I.3) any connected subset of $\sigma$ compatible with $\mathcal{C}_{\sigma}$ is also compatible with $\left\{A_{2}\right\}$.

Before proving this, we remark that (I.3) is equivalent to the following. Let $\Lambda$ be the index set of $\mathcal{C}_{\sigma}$. Let $\mathcal{C}_{\sigma}^{\prime}$ be the family of all connected components of sets in the form $\bigcap_{i \in \Lambda_{0}} \Gamma_{\imath} \cap \bigcap_{\imath \nsubseteq \Lambda_{0}} \Gamma_{i}^{c}, \Lambda_{0}$ being subsets of $\Lambda$ and $\Gamma_{i}^{c}$ denoting the complement in $\sigma$. Then $\mathcal{C}_{\sigma}^{\prime}$ is compatible with $\left\{A_{\imath}\right\}$.

Another remark is that if (I.3) is satisfied for any $m$-simplex of $K$, then the condition (3.1.4) can be replaced by

$(3.1 .4)^{\prime} \quad\left\{\tau(\hat{\sigma}) \mid \sigma \in K^{\prime}\right\} \quad$ is compatible with

$$
\bigcup_{\sigma \in K^{\prime}-K} \mathcal{C}_{\sigma-1} \cup\left\{A_{\imath}\left|A_{i} \subset\right| K^{m-1} \mid\right\} .
$$

Proof of (I). Apply Lemma 3.3 to all $A_{i}$ contained in $\sigma$. Then there exists a point $a_{\sigma} \in \dot{\sigma}$ such that $a_{\sigma} \notin A_{i}$ for any $i$ and that any line through $a_{\sigma}$ is non-singular simultaneously for all $A_{\imath} \subset \sigma$. Now for each $A_{i} \subset \sigma$ and each $b \in A_{i}$, apply Lemma 3.6 to $A_{\imath}, a_{\sigma}$ and $b$. Then we have a compact subanalytic set $B \subset \sum$ and a subanalytic stratification $\left\{B_{j}\right\}$ of $B$ such that $A_{i}$ is a union of some $B_{j}$ 's in a neighborhood $U_{i b}$ of $b$, that each $B_{j}$ is topographic with respect to $\left(\Sigma, a_{\sigma}\right)$ and that $B$ has property $(P)$ with respect to $\left(\Sigma, a_{\sigma}\right)$.

Since $A_{i}$ is compact, we can choose finite $b$ 's so that $U_{i b}$ 's cover $A_{i}$. Gather $B$, for those $b$, and let the family be denoted by $\mathcal{C}_{\sigma i}$. Let $\mathcal{C}_{\sigma}$ be the union of $\mathcal{C}_{\sigma i}$ for all $A_{i} \subset \sigma$. Then $\mathcal{C}_{\sigma}$ clearly is finite and satisfies the properties (I.1,2) except that $\Gamma \subset \sigma$. Moreover, if we replace $\left\{A_{i}\right\}$ in (I.3) by $\left\{A_{i} \mid A_{i} \subset \sigma\right\}$, then (I.3) is satisfied. If $\Gamma \not \subset \sigma$, we consider $\Gamma \cap \sigma^{\circ}$ in place of $\Gamma$. Then the inclusion $\Gamma \subset \sigma$ is satisfied and the other properties remain valid because of $\left\{A_{i}\right\} \supset K^{m-1}$. 
In addition to that, (I.3) in its former condition follows from $\Gamma \subset \sigma$. Hence (I) is proved.

(II) Put $\left\{B_{i}\right\}=\left\{p_{\sigma a_{\sigma}}\left(\Gamma \cap \Gamma^{\prime}\right) \mid \Gamma, \Gamma^{\prime} \in \mathcal{C}_{\sigma}, \sigma \in K-K^{m-1}\right\} \cup\left\{A_{j}\left|A_{j} \subset\right| K^{m-1} \mid\right\}$. Then $\left\{B_{i}\right\}$ is a locally finite family of subanalytic subsets of $\left|K^{m-1}\right|$. Hence there exist by induction hypothesis a subdivision $K^{m-1 \prime}$ of $K^{m-1}$ and a homeomorphism $\pi$ of $\left|K^{m-1}\right|$ such that

(II.1) $\pi$ is subanalytic,

(II.2) $\pi(\sigma)=\sigma$ for any $\sigma \subseteq K^{m-1}$,

(II.3) for any $\sigma \in K^{m-1 \prime}, \pi(\hat{\sigma})$ is an analytic submanifold of $\boldsymbol{R}^{n}$ and $\left.\pi\right|_{\sigma}: \circ \rightarrow \pi(\dot{\sigma})$ is an analytic diffeomorphism and that

(II.4) $\left\{\pi(\stackrel{\circ}{\sigma}) \mid \sigma \in K^{m-1 \prime}\right\}$ is compatible with $\left\{B_{\iota}\right\}$.

We remark that $\left.\pi\right|_{\partial \sigma}$, for any $\sigma \in K$, is a homeomorphism of $\partial \sigma$ because of (II.2). Let $\tilde{\pi}$ be an extension of $\pi$ to $|K|$ defined by

$$
\tilde{\pi}=\left\{\begin{array}{lll}
a_{\sigma} *\left(\left.\pi\right|_{\partial \sigma}\right) & \text { on } & \sigma \in K-K^{m-1} \\
\pi & \text { on } & \left|K^{m-1}\right| .
\end{array}\right.
$$

Then the following are trivial.

(II.5) $\tilde{\pi}$ is a subanalytic homeomorphism of $|K|$ by Lemma 3.7.

(II.6) $\tilde{\pi}(\sigma)=\sigma$ for any $\sigma \in K$.

(II.7) Let $K_{1}^{\prime}$ be the subdivision of $K$

$$
\bigcup_{\sigma \equiv K-K} a_{\sigma-1} * K_{\sigma}^{m-1} \cup K^{m-1}
$$

where

$$
K_{\sigma}^{m-1 \prime}=\left\{\sigma^{\prime} \in K^{m-1^{\prime}} \mid \sigma^{\prime} \subset \sigma\right\}
$$

Then for any $\sigma \in K_{1}^{\prime}, \tilde{\pi}\left({ }^{\circ}\right)$ is an analytic submanifold of $\boldsymbol{R}^{n}$ and $\left.\tilde{\pi}\right|_{\stackrel{\circ}{\sigma}}: \stackrel{\circ}{\sigma} \rightarrow \pi(\stackrel{\circ}{\sigma})$ is an analytic diffeomorphism.

For each $\sigma \in K-K^{n-1}$, put

$$
\begin{aligned}
& \sigma_{[b, c]}=\left\{t\left(x-a_{\sigma}\right)+a_{\sigma} \mid x \in \partial \sigma, b \leqq t \leqq c\right\} \\
& \sigma_{1}=\sigma_{[0,:]}, \sigma_{2}=\sigma_{[\varepsilon, 1]} \quad \text { for } \quad 0<\varepsilon<1,
\end{aligned}
$$

and fix $\varepsilon>0$ so small that $\sigma_{1} \cap \Gamma=\varnothing$ for any $\Gamma \in \mathcal{C}_{\sigma}$. After adding some sets to $\left\{A_{i}\right\}$ and $\mathcal{C}_{\sigma}$, we assume that

(II.8) $\sigma_{1} \cap \sigma_{2}$ is at once an element of $\left\{A_{i}\right\}$ and a union of elements of $\mathcal{C}_{\sigma}$, and $\stackrel{\circ}{\sigma}_{1}$ does not intersect with any $\Gamma \in \mathcal{C}_{\sigma}$.

(III) Let $\sigma$ be an $m$-simplex of $K$, and $\Sigma$ denote the affine space spanned 
by $\sigma$. Put

$$
\begin{aligned}
& \mathcal{K}_{\sigma}=\left\{\pi\left(\dot{\sigma}^{\prime}\right) \mid \sigma^{\prime} \in K_{\sigma}^{m-1^{\prime}}\right\} \\
& \mathcal{L}_{\sigma}=\left\{\text { connected components of } \Gamma \cap p_{\sigma a_{\sigma}}^{-1}(\beta) \mid \Gamma \in \mathcal{C}_{\sigma}, \beta \in \mathcal{K}_{\sigma}\right\} .
\end{aligned}
$$

For any $\beta \in \mathcal{K}_{\sigma}$, denote by $s(\beta)$ the image under $\pi$ of the set of vertexes of $\sigma^{\prime}$ such that $\pi\left(\dot{\sigma}^{\prime}\right)=\beta$. Then the following properties hold.

(III.1) Each $\gamma \in \mathcal{L}_{\sigma}$ is a subanalytic analytic submanifold of $\Sigma$ with $p_{\sigma a_{\sigma}}(\gamma) \in \mathcal{K}_{\sigma}$, topographic with respect to $\left(\Sigma, a_{\sigma}\right)$.

(III.2) $\mathcal{L}_{\sigma}$ is a finite family of disjoint sets.

(III.3) The union of all $\uparrow \in \mathcal{L}_{\sigma}$ coincides with $S_{\sigma}$, hence closed in $\Sigma$ and has property $(P)$.

(III.4) Any connected subset of $\sigma$ compatible with $\mathcal{L}_{\sigma}$ is also compatible with $\left\{A_{i}\right\}$.

(III.5) For any $\gamma \in \mathcal{L}_{\sigma}$, the restriction of $p_{\sigma a_{\sigma}}$ to the closure $\bar{\gamma}$ is a homeomorphism onto $\overline{p_{\sigma a_{\sigma}}(\gamma)}, \bar{\gamma}$ is a union of elements of $\mathcal{L}_{\sigma}$, and we have $\bar{\gamma} \cap p_{\sigma a_{\sigma}}^{-1}(\beta) \in$ $\mathcal{L}_{\sigma}$ for any $\beta \in \mathcal{K}_{\sigma}$ contained in $\overline{p_{\sigma a_{\sigma}}(\gamma)}$.

Proof. If $\gamma$ is a connected component of $\Gamma \cap p_{\sigma a}^{-1}(\beta)$ for $\Gamma \in \mathcal{C}_{\sigma}, \beta \in \mathcal{K}_{\sigma}$, then we have by (II.4) $\beta \subset p_{\sigma a_{\sigma}}(\Gamma)$ which, together with (I.1) and (II.1,3), implies the first half of (III.1). As to (III.2), the finiteness of $\mathcal{L}_{\sigma}$ is trivial by (2.2). If $\gamma_{i}, i=1,2$, are respective connected components of $\Gamma_{i} \cap p_{\sigma a}^{-1}\left(\beta_{i}\right)$ with $\gamma_{1} \cap \gamma_{2} \neq \varnothing$, then $\beta_{1} \cap \beta_{2} \cap p_{\sigma a_{\sigma}}\left(\Gamma_{1} \cap \Gamma_{2}\right) \neq \varnothing$, hence $\beta_{1}=\beta_{2} \subset p_{\sigma a_{\sigma}}\left(\Gamma_{1} \cap \Gamma_{2}\right)$ by (II.4), which gives $\gamma_{1}=\gamma_{2}$. The property (III.3) is trivial by (I.2), and (III.4) is an easy consequence of (I.3) since any $\Gamma \in \mathcal{C}_{\sigma}$ is a union of some elements of $\mathcal{L}_{\sigma}$.

To prove (III.5), let $\gamma \in \mathcal{L}_{\sigma}, \beta=p_{\sigma a_{\sigma}}(\gamma) \in \mathcal{K}_{\sigma}$. We have $p_{\sigma a_{\sigma}}(\bar{\gamma})=\bar{\beta}$ because of $\gamma \subset \sigma_{2}$, and $\bar{\gamma} \subset S_{\sigma}$ by (III.3). For any $x \in \bar{\beta}-\beta$, the subanalytic set $\bar{\gamma} \cap p_{\sigma a}^{-1}(x)$ has dimension 0 by (I.1) and hence is discrete. This easily implies that $\left.p_{\sigma a_{\sigma}}\right|_{\bar{\gamma}}$ : $\bar{\gamma} \rightarrow \bar{\beta}$ is a homeomorphism (the first part of III.5). Let $\beta_{1} \in \mathcal{K}_{\sigma}$ with $\beta_{1} \subset \bar{\beta}$. Then $\bar{\gamma} \cap p_{\sigma a_{\sigma}}^{-1}\left(\beta_{1}\right)$ is homeomorphic to $\beta_{1}$ under $p_{\sigma a_{\sigma}}$, and it is contained in $S_{\sigma} \cap p_{\sigma a_{\sigma}}^{-1}\left(\beta_{1}\right)$, which is a finite union of disjoint elements of $\mathcal{L}_{\sigma}$, each being homeomorphic to $\beta_{1}$ under $p_{\sigma a_{\sigma}}$. Hence $\bar{\gamma} \cap p_{\sigma a_{\sigma}}^{-1}\left(\beta_{1}\right)$ must be one of those elements. This establishes the second and third parts of (III.5).

Considering the barycentric subdivision $\operatorname{Sd}\left(K^{m-1 \prime}\right)$ of $K^{m-1 \prime}$ in place of $K^{m-1 \prime}$ if necessary, we can assume

(III.6) Let $\gamma_{1}, \gamma_{2} \in \mathcal{L}_{\sigma}$ with $p_{\sigma a_{\sigma}}\left(\gamma_{1}\right)=p_{\sigma a_{\sigma}}\left(\gamma_{2}\right)=\beta$. Assume $\gamma_{1}<\gamma_{2}$ with respect to $\left(\Sigma, a_{\sigma}\right)$. Then $\bar{\gamma}_{1} \leqq \bar{\gamma}_{2}$ with respect to $\left(\Sigma, a_{\sigma}\right)$ and

$$
\bar{\gamma}_{1} \cap p_{\sigma a}^{-1}(s(\beta)) \neq \bar{\gamma}_{2} \cap p_{\sigma a}^{-1}(s(\beta)) .
$$

The reason is the following. It is clear that $\bar{\gamma}_{1} \leqq \bar{\gamma}_{2}$ with respect to $\left(\Sigma, a_{\sigma}\right)$. 
Let $\gamma_{i} \subset \Gamma_{i}$ with $\Gamma_{i} \in \mathcal{C}_{\sigma}, i=1,2, \beta=\pi\left(\dot{\sigma}^{\prime}\right)$ with $\sigma^{\prime} \in \operatorname{Sd}\left(K_{\sigma}^{m-1^{\prime}}\right)$ and $\dot{\sigma}^{\prime} \subset \tilde{\tilde{\sigma}}^{\prime}$ with $\tilde{\sigma}^{\prime} \in K_{\sigma}^{m-1 \prime}$. Let $\tilde{\gamma}_{i}, i=1,2$, be the connected components of $\Gamma_{i} \cap p_{\sigma a}^{-1}\left(\pi\left(\tilde{\sigma}^{\prime}\right)\right)$ containing $\gamma_{i}$. Then, using (II.3) we see in the same way as the above proof that

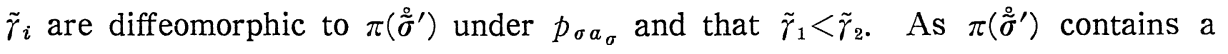
point of $s(\beta)$, that implies the required inequality.

Let $\gamma_{1}, \gamma_{2} \in \mathcal{L}_{\sigma}$. We call $\gamma_{1}, \gamma_{2}$ a consecutive couple of $\mathcal{L}_{\sigma}$ if $p_{\sigma a_{\sigma}}\left(\gamma_{1}\right)=p_{\sigma a_{\sigma}}\left(\gamma_{2}\right)$, $\gamma_{1}<\gamma_{2}$ with respect to $\left(\Sigma, a_{\sigma}\right)$ and if $\left(\gamma_{1}, \gamma_{2}\right)$ does not contain any element of $\mathcal{L}_{\sigma}$. Then we have

(III.7) Let $\gamma_{1}, \gamma_{2}$ be a consecutive couple of $\mathcal{L}_{\sigma}$. For each $\beta \in \mathcal{K}_{\sigma}$ contained in $\overline{p_{\sigma a_{\sigma}}\left(\gamma_{1}\right)}, \bar{\gamma}_{1} \cap p_{\sigma a_{\sigma}}^{-1}(\beta)$ and $\bar{\gamma}_{2} \cap p_{\sigma a_{\sigma}}^{-1}(\beta)$ are identical or consecutive.

In fact $\gamma_{i}^{\prime}=\bar{\gamma}_{i} \cap p_{\sigma a_{\sigma}}^{-1}(\beta), i=1,2$, are elements of $\mathcal{L}_{\sigma}$ (III.5). Hence $\gamma_{\mathfrak{l}}^{\prime}=\gamma_{2}^{\prime}$ or $\gamma_{1}^{\prime}<\gamma_{2}^{\prime}$ with respect to $\left(\Sigma, a_{\sigma}\right)$. Assume that an element of $\mathcal{L}_{\sigma}$ is contained in $\left(\gamma_{1}^{\prime}, \gamma_{2}^{\prime}\right)$. Then, since $S_{\sigma}$ has property $(P)$ (III.3), we have $S_{\sigma} \cap\left(\gamma_{1}, \gamma_{2}\right) \neq \varnothing$. As $S_{\sigma} \cap p_{\sigma a_{\sigma}}^{-1}\left(p_{\sigma a_{\sigma}}\left(\gamma_{1}\right)\right)$ is a union of some elements of $\mathcal{L}_{\sigma}$, that implies $\gamma_{3} \subset\left(\gamma_{1}, \gamma_{2}\right)$ for some $\gamma_{3} \in \mathcal{L}_{\sigma}$, which is a contradiction.

Denote by $\mathcal{L}_{\sigma}^{\#}$ the family of all sets $\left(\gamma_{1}, \gamma_{2}\right)$ where $\gamma_{1}, \gamma_{2}$ are consecutive couples of $\mathcal{L}_{\sigma}$. Put

$$
\mathcal{L}_{\sigma}^{*}=\mathcal{L}_{\sigma} \cup \mathcal{L}_{\sigma}^{\#}
$$

Then we have

(III.8) $\mathcal{L}_{\sigma}^{*}$ is a finite family of disjoint subanalytic analytic submanifolds of $\Sigma$, whose union is equal to $\sigma_{2}$ (in II).

Indeed the first half follows from (III.1,2), and the latter half does from (I.1), (II.8), (IIII.3) and the inclusion $\left\{A_{i}\right\} \supset K^{m-1}$.

By (III.4)

(III.9) $\mathcal{L}_{\sigma}^{*}$ is compatible with $\left\{A_{\imath}\right\}$.

Finally it follows from (III.2,5,7) that

(III.10) For any $\gamma \in \mathcal{L}_{\sigma}^{*}, \bar{\gamma}$ is a union of some elements of $\mathcal{L}_{\sigma}^{*}$. If $\gamma=\left(\gamma_{1}, \gamma_{2}\right) \in$ $\mathcal{L}_{\sigma}^{*}$, any element of $\mathcal{L}_{\sigma}^{*}$ contained in $\bar{\gamma}$ is in the form $\bar{\gamma}_{1} \cap p_{\sigma a_{\sigma}}^{-1}(\beta), \bar{\gamma}_{2} \cap p_{\sigma a_{\sigma}}^{-1}(\beta)$ or $\left(\bar{\gamma}_{1} \cap p_{\sigma a_{\sigma}}^{-1}(\beta), \bar{\gamma}_{2} \cap p_{\sigma a_{\sigma}}^{-1}(\beta)\right)$ with $\beta \in \mathcal{K}_{\sigma}, \beta \subset \overline{p_{\sigma a_{\sigma}}(\gamma)}$.

(IV) We use the same notations as (III). For any $\gamma \in \mathcal{L}_{\sigma}$, we define a simplex $\rho(\gamma) \subset \sigma$ as follows. Put $\beta=p_{\sigma a_{\sigma}}(\gamma)$. Consider $\tilde{\pi}^{-1}\left(\bar{\gamma} \cap p_{\sigma a}^{-1}(s(\beta))\right)$. It consists of indepentdent ( $\operatorname{dim} \gamma+1)$-points by (III.5) (see [13] for the definition of independence), because the image of the set under $p_{\sigma a_{\sigma}}$ is $\pi^{-1}(s(\beta))$. Let $\rho(\gamma)$ be the simplex spanned by the set, and $\rho(\gamma)$ be the open simplex. Then we have

$$
p_{\sigma a_{\sigma}}(\hat{\rho}(\gamma))=\pi^{-1}\left(p_{\sigma a_{\sigma}}(\gamma)\right)
$$


since

$$
\dot{\varphi}^{-1} \circ \tilde{\pi} \circ \phi(x, t)=(\pi(x), t) \quad \text { for } \quad(x, t) \in \phi^{-1}(\gamma)
$$

where the subanalytic homeomorphism $\psi: \partial \sigma \times(0,1] \rightarrow \sigma-a_{\sigma}$ is defined by

$$
\phi(x, t)=t\left(x-a_{\sigma}\right)+a_{\sigma} .
$$

(IV.2) If $i<\gamma^{\prime}$ with respect to $\left(\Sigma, a_{\sigma}\right)$, then $\hat{\rho}(\gamma)<\stackrel{\rho}{\rho}\left(\gamma^{\prime}\right)$ with respect to $\left(\Sigma, a_{\sigma}\right)$ by (III.6),

(IV.3) If $\gamma^{\prime} \subset_{\bar{i}}$ then $\rho\left(\gamma^{\prime}\right)$ is a face of $\rho(\gamma)$ by (III.5), and

(IV.4) If $\gamma \in \mathcal{L}_{\sigma}$ is contained in $\partial \sigma$ or $\partial \sigma_{1}$, then $\rho(\gamma)=\tilde{\pi}^{-1}(\gamma)$ by III.8), where $\sigma_{1}$ is defined in (II).

Put

$$
\begin{aligned}
& L_{\sigma}=\left\{\rho(\gamma) \mid \gamma \in \mathcal{L}_{\sigma}\right\}, \\
& L_{i \sigma_{1}}=\left\{\rho(\gamma) \mid \gamma \in \mathcal{L}_{\sigma}, \gamma \subset \partial \sigma_{1}\right\}, \\
& L_{\sigma}^{\sharp}=\left\{\left[\rho(\gamma), \rho\left(\gamma^{\prime}\right)\right] \mid \gamma, \gamma^{\prime}: \text { consecutive couples of } \mathcal{L}_{\sigma}\right\} . \\
& L_{\sigma}^{*}=L_{\sigma} \cup a_{\sigma} * L_{\partial \sigma_{1}} \cup L_{\sigma}^{\ddagger} .
\end{aligned}
$$

Then, by (III.8, 10) and (IV.1, 2, 3, 4)

(IV.5) $L_{\sigma}^{*}$ is a finite cell complex whose underlying polyhedron is equal to $\sigma$.

Let $\tau_{\sigma}$ be a homeomorphism of $\sigma$ defined so that

$$
\begin{aligned}
& \sigma_{\sigma}(\stackrel{\rho}{\rho}(\gamma))=\gamma \quad \text { for } \gamma \in \mathcal{L}_{\sigma}, \\
& \tau_{\sigma}\left(\left(\stackrel{\circ}{\rho}\left(\gamma_{1}\right), \stackrel{\circ}{\rho}\left(\gamma_{2}\right)\right)=\left(\gamma_{1}, \gamma_{2}\right) \text { for }\left(\gamma_{1}, \gamma_{2}\right) \in \mathcal{L}_{\sigma}^{\#},\right. \\
& p_{\sigma a_{\sigma}}{ }^{\circ} \tau_{\sigma}=\pi \circ p_{\sigma a_{\sigma}} \text { on } \sigma_{2}, \\
& \tau_{\sigma}=\tilde{\pi} \quad \text { on } \sigma_{1},
\end{aligned}
$$

and that for any $\left(\gamma_{1}, \gamma_{2}\right) \in \mathcal{L}_{\sigma}^{\sharp}$ and any $x \in \partial \sigma, \tau_{\sigma}$ is linear on the segment $\left(\dot{\rho}\left(\gamma_{1}\right)\right.$, $\left.\vec{\rho}\left(\gamma_{2}\right)\right) \cap p_{\sigma a}^{-1}(x)$. Then $\tau_{\sigma}$ is uniquely well-defined and equal to $\tau$ on $\partial \sigma$ by the definition of $\approx$, (III.8, 10) and (IV.4).

Hence we have a homeomorphism $\tau$ of $|K|$ whose restriction to each $\sigma$ coincides with $\tau_{\sigma}$. Letting $K^{\prime}$ be a simplicial subdivision of $\sum_{\sigma \equiv K-K^{n-2}}^{1} L_{\sigma}^{*} \cup K^{m-1}$, we want to see that $\tau, K^{\prime}$ satisfy $(3.1 .1,2,3,4)$. First note that $(3.1 .1$ is equivalent to (IV.6) $\left.\tau_{\sigma}\right|_{\sigma^{\prime}}$ is subanalytic for each $\sigma^{\prime} \in L_{\sigma}^{*}$.

Proof of (IV.6). If $\sigma^{\prime} \subset \sigma_{1}$ it follows from (II.5) and from $\tau_{j}=\pi$ on $\sigma^{\prime}$. Assume $\sigma^{\prime}=o\left(\gamma^{\prime}\right)$ with $r \in \mathcal{L}_{\sigma}$. Then

$$
\left.\tau_{\sigma}\right|_{\sigma^{\prime}}=\left.\left(\left.p_{\sigma a_{\sigma}}\right|_{\bar{\gamma}}\right)^{-1} \circ \pi \circ p_{\sigma a_{\sigma}}\right|_{\sigma^{\prime}} \cdot
$$

Hence (2.13.3) shows that $\left.\tau_{\sigma}\right|_{\sigma^{\prime}}$ is subanalytic. Let $\sigma^{\prime}=\left[\rho(\gamma), \rho \eta^{\prime}-\right.$ with $\gamma, \gamma^{\prime}$ 
$\in \mathcal{L}_{\sigma}$. Then, by the above statement and (2.4) we have compact analytic manifolds $M, M^{\prime}$ and analytic maps $f=\left(f_{1}, f_{2}\right): M \rightarrow \sigma \times \sigma, f^{\prime}=\left(f_{1}^{\prime}, f_{2}^{\prime}\right): M^{\prime} \rightarrow \sigma \times \sigma$ such that $f(M)=\left.\operatorname{graph} \tau_{\sigma}\right|_{\rho(\gamma)}, f\left(M^{\prime}\right)=$ graph $\left.\tau_{\sigma}\right|_{\rho\left(i^{\prime}\right)}$. Define an analytic map $F: M \times M^{\prime} \times[0,1] \rightarrow \sigma \times \sigma$ by

$$
\begin{aligned}
F\left(x, x^{\prime}, t\right) & =t f(x)+(1-t) f^{\prime}\left(x^{\prime}\right) \\
& =\left(t f_{1}(x)+(1-t) f_{1}^{\prime}\left(x^{\prime}\right), t f_{2}(x)+(1-t) f_{2}^{\prime}\left(x^{\prime}\right)\right) .
\end{aligned}
$$

Put

$$
M^{\prime \prime}=\left\{\left(x, x^{\prime}\right) \in M \times M M^{\prime} \mid p_{\sigma a_{\sigma}} \circ f_{1}(x)=p_{\sigma a_{\sigma}} \circ f_{1}^{\prime}\left(x^{\prime}\right)\right\} .
$$

Then $M^{\prime \prime}$ is a compact analytic set, and we have

$$
F\left(M^{\prime \prime} \times[0,1]\right)=\left.\operatorname{graph} \tau_{\sigma}\right|_{\sigma^{\prime}} .
$$

Hence, by (2.3) and (2.5.2), graph $\left.\tau_{\sigma}\right|_{\sigma^{\prime}}$ is subanalytic which proves (IV.6).

$(3.1 .2,3)$ are trivial, and (3.1.4) follows from (II.8) and (III.9). Hence we complete the proof of Proposition 3.1'.

Remark 3.8. We can refine Propositions $3.1,1^{\prime}$ as follows. Let $L$ be a subcomplex of $\AA^{\circ}$. Assume $\{\sigma \mid \sigma \in K-L\}$ is compatible with $\left\{A_{\imath}\right\}$. Then $\tau$ can satisfy moreover, for any $\sigma \in K$ with $\sigma \cap|L|=\varnothing$,

$$
\begin{aligned}
& \tau=\text { ideni } \\
& \sigma \in H^{-1} .
\end{aligned}
$$

It is clear by the method of construction of $\tau$ in the proof of Proposition 3.1'.

The condition (3.1.5) will be important when we will enlarge the domain where $f$ is piecewise linearized in the proof of Theorem I, $\S 7$. This is one of the reasons why we refined a result of [4].

We will show a semi-algebraic version of Propositions 3.1, 1'. A subset of $R^{n}$ is called semi-algebraic if it is a finite union of sets in the form

$$
\left\{x \in R^{n} \mid f_{1}(x)>0, \cdots, f_{k}(x)>0, f_{k+1}(x)=0, \cdots, f_{l}(x)=0\right\}
$$

where $f_{i}$ are polynomials on $\boldsymbol{R}^{n}$. A continuous map between semi-algebraic sets is called semi-algebraic if the graph is semi-algebraic. We remark that the semi-algebraic rersions of the results in Section 2 hold true, in which the locally finite condition must be replaced by the finite one (see [1], [4], [10]). Since the stereographic projection $\boldsymbol{R}^{n} \rightarrow S^{n}$ is semi-algebraic, in most problems of semialgebraic sets we can restrict the sets to being bounded.

Proposition 3.9. Let $K$ and $L$ be a finite simplicial complex in $\boldsymbol{R}^{n}$ and $a$ subcomplex respectively. Let $\left\{A_{i}\right\}$ be a finite family of bounded semi-algebraic subsets of $\mathbb{R}^{n}$ contained in $|K|$ such that $\{\dot{\sigma} \mid \sigma \in K-L\}$ is compatible with $\left\{A_{i}\right\}$. Then there exist a subdivision $K^{\prime}$ of $K$ and a homeomorphism $\tau$ of $\boldsymbol{R}^{n}$ such that 
(3.9.1) = is semi-algebraic,

(3.9.2) $\tau(\sigma)=\sigma$ for any $\sigma \in K$,

(3.9.3) for any $\sigma \in K^{\prime}, \tau\left(\sigma^{\circ}\right)$ is an analytic submanifold of $\boldsymbol{R}^{n}$ anci $=^{\prime}: \dot{\sigma} \rightarrow \tau(\dot{\sigma})$ is an analytic diffeomorphism,

(3.9.4) $\left\{\tau(\tilde{\sigma}) \mid \sigma \in K^{\prime}\right\}$ is compatible with $\left\{\mathcal{A}_{2}\right\}$,

(3.9.5) $\tau=$ ident on any $\sigma \in K$ with $\sigma \cap|L|=\varnothing$ and that

(3.9.6) any $\sigma \in K$, with $\sigma \cap|L|=\varnothing$, is a simplex of $K^{\prime}$.

This is a refinement of a theorem of [4] and Theorem 3, - 10]. As we can prove it in the same way as Proposition 3.1', we omit the proor.

Remark 3.10. In propositions 3.1 and $3.1^{\prime}$ or $3.9, \tau$ is subanalytically or semi-algebraically isotopic to the identity respectively. Namely there exists an isotopy $\tau_{t}:|K| \rightarrow|K|, 0 \leqq t \leqq 1$, such that $\tau_{0}=\tau, \tau_{1}=$ ident and that the map $T$ : $|K| \times I \rightarrow|K| \times I, I=[0,1]$, defined by $T(x, t)=\left(\tau_{t}(x), t\right)$ is subanalytic or semialgebraic respectively. Moreover we can choose the isotopy so that for any $\sigma \in K^{\prime}$, the restriction of $T^{-1}$ to $\tau(\stackrel{\sigma}{\sigma}) \times I$ is an analytic diffeomorphism. This is also clear by the method of construction of $\tau$.

\section{$\S 4$. Whitney Stratifications and Vector Fields}

In this section, we prepare for the proof of Proposition 5.1. All results are derived from the Thom-Mather theory of Whitney stratification. The result which will be applied is only Lemma 4.14, and the others need for its proof. Manifolds, vector bundles and maps are of class $C^{\infty}$, and we do not necessarily assume the frontier condition (2.9.2), the connectedness of strata, nor the boundedness for the definition of stratification. Let us recall some definitions (see [1]).

Definition 4.1. Let $X \subset \boldsymbol{R}^{n}$ be a submanifold. A tube at $I$ is a quadruple $T=(E, \pi, \rho, e)$ where $\pi: E \rightarrow Y$ is a vector bundle of $\operatorname{dimension}=\operatorname{codim} X, \rho$ : $E \rightarrow \boldsymbol{R}$ is the quadratic function of a Riemannian metric on $E$. and $e: E_{0} \rightarrow \boldsymbol{R}^{n}$ is an imbedding, commuting with the zero section $\zeta: X \rightarrow E$ so that $e \circ \zeta$ is the inclusion $X \subset R^{n}, E_{0}$ being an open neighborhood of $\zeta(X)$. We set $|T|=e\left(E_{n}\right)$. By identifying $E_{0}$ with $|T|$, we use $\pi, \rho$ also as the map $\pi \circ \epsilon^{-1}$ and the function $\rho \circ e^{-1}$ on $|T|$ respectively. For any open subset $X^{\prime}$ of $X, T_{\text {I }}$ means $\left(\pi^{-1}\left(X^{\prime}\right)\right.$,

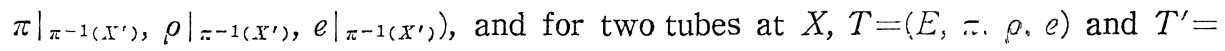
$\left(E^{\prime}, \pi^{\prime}, \rho^{\prime}, e^{\prime}\right)$, we write as $T \equiv T^{\prime}$ if we have a neighborhood $L_{i}$ of $X$ in $R^{n}$ such that $U \subset|T| \cap\left|T^{\prime}\right|,\left.\pi\right|_{U}=\left.\pi^{\prime}\right|_{U}$ and $\left.\rho\right|_{U}=\left.\rho^{\prime}\right|_{U}$.

Definition 4.2. Let $\left\{X_{i}\right\}$ be a Whitney stratification of a subset $X$ of $R^{n}$. A tube system for $\left\{X_{i}\right\}$ consists of one tube $T_{\imath}=\left(E_{i}, \pi_{i}, \rho_{l}, c_{i}\right)$ at each $X_{i}$. The tube system is called weakly controlled if 


$$
\pi_{2} \circ \pi_{\jmath}(x)=\pi_{i}(x), \quad x \in\left|T_{i}\right| \cap\left|T_{\jmath}\right| \cap \pi_{j}^{-1}\left|T_{i}\right| .
$$

We call it controlled if furthermore

$$
\rho_{\imath} \circ \pi_{j}(x)=\rho_{i}(x), \quad x \in\left|T_{i}\right| \cap\left|T_{j}\right| \cap \pi_{j}^{-1}\left|T_{i}\right| .
$$

Definition 4.3. Let $\left\{X_{i}\right\},\left\{Y_{j}\right\}$ be Whitney stratifications of subsets $X, Y$ of $\boldsymbol{R}^{n}, \boldsymbol{R}^{m}$ respectively. We call a $C^{\infty}$ map $f$ from $\boldsymbol{R}^{n}$ to $\boldsymbol{R}^{m}$ a Thom map with respect to $\left\{X_{2}\right\} .\left\{Y_{j}\right\}$ when for any $X_{i}, X_{i}$, the following are satisfied.

(4.3.1) $f$ maps $Y_{\iota}, X_{i^{\prime}}$ submersively into some strata (not necessarily onto).

(4.3.2) Let $\left\{x_{3}\right\}$ be a sequence of points in $X_{i}$ converging to $x \in X_{i}$, such that $\left\{\text { ker } d\left(\left.f\right|_{x_{i}}\right)_{x_{k}}\right\}_{k}$ converges, in the appropriate Grassmann bundle, to a plane $L \subset \boldsymbol{R}^{n}$. Then ker $d\left(\left.f\right|_{i^{\prime}}\right)_{x} \subset L$.

Definition 4.4. Let $\left\{X_{i}\right\},\left\{Y_{j}\right\}$ be Whitney stratifications of subsets $X, Y$ of $\boldsymbol{R}^{n}, \boldsymbol{R}^{m}$ respectively, and $f: \boldsymbol{R}^{n} \rightarrow \boldsymbol{R}^{m}$ be a Thom map with respect to $\left\{X_{\imath}\right\}$, $\left\{Y_{j}\right\}$. A tube system $\left\{T_{i}\right\}$ for $\left\{X_{i}\right\}$ is controlled over a tube system $\left\{R_{j}\right\}$ for $\left\{Y_{j}\right\}$ if $\left\{T_{i}\right\}$ satisfies (4.2.1) and (4.4.1) below and if (4.2.2) holds for any strata $X_{i}, X_{i^{\prime}}$ with $f\left(X_{i}\right) \cup f\left(X_{i^{\prime}}\right) \subset Y_{j}$ for some $j$.

(4.4.1) For any $I_{l}, Y_{j}$ with $f\left(X_{i}\right) \subset Y_{j}$

$$
f \circ \pi_{i}(x)=\pi_{j} \circ f(x), \quad x \in\left|T_{i}\right| \cap f^{-1}\left|R_{\jmath}\right| .
$$

Definition 4.5. Let $M, N \subset \boldsymbol{R}^{n}$ be $C^{\infty}$ manifolds with $M \cap N^{\top}=\varnothing$. We say that $(M, N)$ satisfies the Whitney condition (a) if the following is satisfied.

(4.5.1) Let $\left\{x_{2}\right\}$ be a sequence of points in $M$ converging to $x \in N^{\top}$. If the sequence of tangent spaces $\left\{T_{x_{i}} M\right\}$ converges to a subspace $L \subset \boldsymbol{R}^{n}$, then $L \supset T_{x} N$.

Remark 4.6. If $(M, N)$ satisfies the Whitney condition, then it does the Whitney condition (a) (see [11]).

Lemma 4.7. Let $\left\{X_{i}\right\},\left\{Y_{j}\right\}$ be Whitney stratifications of subsets $X, Y$ of $\boldsymbol{R}^{n}, \boldsymbol{R}^{m}$ respectively, and $f: \boldsymbol{R}^{n} \rightarrow \boldsymbol{R}^{m}$ be a Thom map with respect to $\left\{X_{i}\right\},\left\{Y_{j}\right\}$. Then for any weakly controlled tube system $\left\{R_{j}\right\}$ for $\left\{Y_{j}\right\}$ there exists a tube system $\left\{T_{i}\right\}$ for $\left\{X_{2}\right\}$ which is controlled over $\left\{R_{j}\right\}$. Moreover, if $\left\{T_{\imath}^{0}\right\}$ is a tube system for $\left\{X_{2}, U\right\}$ controlled over $\left\{R_{j}\right\}, U$ being an open set in $\boldsymbol{R}^{n}$, then $\left\{T_{i}\right\}$ can be chosen so that $\left.\left.T_{i}\right|_{U^{\prime} \cap X_{\imath}} \equiv T_{\imath}^{0}\right|_{U^{\prime} \cap x_{i}}$ for any given open set $U^{\prime} \subset \boldsymbol{R}^{n}$ with $\bar{U}^{\prime}$ $-U \subset \bar{X}-X$.

Proof. The first half coincides with Theorem 2.6, Chapter II, [1], and the latter half is clear from the construction of $\left\{T_{i}\right\}$ in its proof. So we omit the proof.

Corollary 4.8. Let $\left\{X_{i}\right\}_{\imath=1,2, \ldots}$ be a Whitney stratification of a subset $X$ of $\boldsymbol{R}^{n}$. Let $V$ be an open neighborhood of $X_{1}$ in $\boldsymbol{R}^{n}$, and $\pi: V \rightarrow X_{1}$ be a submersion 
with $\left.\pi\right|_{x_{1}}=$ ident. Then there exist an open neighborhood $V^{\prime} \subset V$ of $X_{1}$ and $a$ controlled tube system $\left\{T_{i}=\left(E_{i}, \pi_{i}, \rho_{i}, e_{i}\right)\right\}$ for $\left\{X_{\imath}^{\prime}=X_{\imath} \cap V^{\prime}\right\}$ such that $\left|T_{1}\right| \subset V$ and $\pi_{1}=\pi$ on $\left|T_{1}\right|$. Moreover if $\left\{T_{i}^{0}=\left(E_{\imath}^{0}, \pi_{\imath}^{0}, \rho_{l}^{0}, e_{\imath}^{0}\right)\right\}$ is a controlled tube system for $\left\{X_{i} \cap U\right\}$ such that $\pi_{1}^{0}=\pi$ on $\left|T_{1}^{0}\right| \cap V, U$ being an open set in $\boldsymbol{R}^{n}$, then $\left\{T_{i}\right\}$ can be chosen so that $\left.\left.T_{i}\right|_{V^{\prime} \cap U^{\prime} \cap_{1} X_{\imath}} \equiv T_{\imath}^{0}\right|_{V^{\prime} \cap U^{\prime} \cap X_{2}}$ for any given open set $U^{\prime}$ with $\overline{V \cap U^{\prime}}-U \subset \bar{X}_{1}-X_{1}$.

Proof. As $\left(\boldsymbol{R}^{n}-\bar{X}, X_{\imath}\right)$ satisfies the Whitney condition for any $i$, we can add $\boldsymbol{R}^{n}-\bar{X}$ to $\left\{X_{i}\right\}$. Hence we may assume $\bar{X}=\boldsymbol{R}^{n}$. By Remark 4.6 there exists an open neighborhood $V^{\prime} \subset V$ of $X_{1}$ such that for any $x \in X_{1}$,

$\pi^{-1}(x)$ and $X_{\imath}^{\prime}$ are transversal, $i=1,2, \cdots$.

Put $\left\{Y_{j}\right\}=\{Y\}=\left\{X_{1}\right\}, \boldsymbol{R}^{m}=\boldsymbol{R}^{n}$, and let $f$ be any $C^{\infty}$ extension of $\pi: V^{\prime} \rightarrow Y$ to $\boldsymbol{R}^{n} \rightarrow \boldsymbol{R}^{n}$. Then if tube systems $\left\{T_{i}=\left(E_{i}, \pi_{i}, \rho_{l}, e_{i}\right)\right\}$ for $\left\{X_{i}^{\prime}\right\}$ and $\{R\}$ for $\{Y\}$ satisfy (4.4.1), then we have $\pi_{1}=\pi$ on $\left|T_{1}\right|$ because of $\bar{X}=\boldsymbol{R}^{n}$, and if $\left\{T_{i}\right\}$ is controlled over $\{R\},\left\{T_{i}\right\}$ itself is controlled. Hence Corollary 4.8 follows from Lemma 4.7 if we see that $f$ is a Thom map with respect to $\left\{X_{i}^{\prime}\right\}$ and $\{Y\}$.

Let $X_{i}, X_{i^{\prime}}$ be any strata. It is clear by (4.8.1) that $\pi\left|x_{\imath}^{\prime}, \pi\right|_{x_{\imath}^{\prime}}$ are submersions into $Y$ (4.3.1). Let $\left\{x_{j}\right\}$ be a sequence of points in $X_{i}^{\prime}$ converging to $x \in X_{i}^{\prime}$, such that $\left\{\operatorname{ker} d\left(\left.\pi\right|_{X_{i}^{\prime}} ^{\prime}\right)_{x}\right\}_{j}$ converges to a plane $L \subset \boldsymbol{R}^{n}$. We want to see $\operatorname{ker} d\left(\left.\pi\right|_{X^{\prime}} ^{\prime}\right)_{x} \subset L$. This follows from the following facts.

(4.8.2) We can assume that $\left\{T_{x_{j}} X_{i}^{\prime}\right\}_{j}$ converges to a plane in $\boldsymbol{R}^{n}$.

(4.8.3) We have $\operatorname{ker} d\left(\left.\pi\right|_{x_{i}^{\prime}}\right)_{x_{j}}=T_{x_{j}} X_{i}^{\prime} \cap T_{x_{j}} \pi^{-1}\left(\pi\left(x_{j}\right)\right)$.

(4.8.4) Let $\left\{K_{j}\right\}\left\{L_{j}\right\}$ be sequences of linear subspaces of $\boldsymbol{R}^{n}$ converging to $K$, $L$ respectively such that $K, L$ are transversal. Then, for sufficiently large $j, K_{j}$ and $L_{j}$ are transversal, and $\left\{K_{j} \cap L_{j}\right\}$ converges to $K \cap L$.

Thus we saw that $f$ is a Thom map with respect to $\left\{X_{\imath}^{\prime}\right\}$ and $\{Y\}$, which proves the lemma.

We regard $\boldsymbol{R}^{l}, l=1, \cdots, n$, as linear subspaces

$$
\left\{\left(x_{1}, \cdots, x_{n}\right) \in \boldsymbol{R}^{n} \mid x_{l+1}=\cdots=x_{n}=0\right\}
$$

of $\boldsymbol{R}^{n}$, and we put

$$
x+\boldsymbol{R}^{l}=\left\{x+y \mid y \in \boldsymbol{R}^{l}\right\} \quad \text { for } \quad x \in \boldsymbol{R}^{n} .
$$

Lemma 4.9. Let $\left\{X_{i}\right\}$ be a Whitney stratification of a subset $X$ of $\boldsymbol{R}^{n}$, and $U_{i}$ be an open neighborhood of each $X_{i}$ in $\boldsymbol{R}^{n}$. Assume that for each $X_{i}$ and $x$ $\in \boldsymbol{R}^{n}, x+\boldsymbol{R}^{l}$ and $X_{i}$ are transversal where $l=\operatorname{codim} X_{i}$. Then there exists a controlled tube system $\left\{T_{i}\right\}$ for $\left\{X_{i}\right\}$ such that for each $i$

$$
\left|T_{\iota}\right| \subset U_{\imath} \text { and }
$$




$$
\begin{gathered}
\pi_{i}^{-1}(x) \subset x+\boldsymbol{R}^{n-m} \quad \text { for } x \in X_{i} \cap\left(\bigcup_{j \in I m} U_{j}-\bigcup_{j \in I m-1} U_{j}\right), \\
m=0, \cdots, \operatorname{dim} X_{i},
\end{gathered}
$$

where $I_{j}, j=0, \cdots, n$, are the index subsets consisting of $i$ with $\operatorname{dim} X_{i} \leqq j$, and $I_{-1}=\varnothing$.

Proof. Considering $\left\{\bigcup_{\operatorname{dim}} \bigcup_{i=j} X_{i}\right\}_{j=0,1, \ldots}$ in place of $\left\{X_{i}\right\}$, we can assume $\left\{X_{i}\right\}=\left\{X_{i}\right\}_{i=0, \ldots}$ and $\operatorname{dim} X_{i}=i$. Put $Y_{j}=\bigcup_{i=0}^{j} X_{i}$. We prove the lemma inductively as follows; assume that we have already a controlled tube system $\left\{T_{i}^{\prime}=\left(E_{i}^{\prime}, \pi_{i}^{\prime}\right.\right.$, $\left.\left.\rho_{i}^{\prime}, e_{i}^{\prime}\right)\right\}$ for $\left\{X_{i} \cap V_{j-1}\right\}$ which satisfy $(4.9 .1,2)$ where $V_{j-1}$ is an open neighborhood of $Y_{j-1}$ contained in $\bigcup_{k=0}^{j-1} U_{k}$ (the case $j=0$ is trivial). Here we remark that $U_{j}, U_{j+1}, \cdots$ are not necessary when we see (4.9.2). Later we will replace $U_{1}, \cdots$, $U_{j-1}, V_{j-1}$ by smaller ones. Hence the induction hypothesis is settled for any $U_{1}, \cdots, U_{j-1}$. After defining $V_{j}$, we want to extend $\left\{T_{\imath}^{\prime}\right\}$ to a controlled tube system for $\left\{X_{i} \cap V_{j}\right\}$.

Let $T_{j 1}=\left(E_{j 1}, \pi_{j 1}, \rho_{j 1}, e_{j 1}\right)$ be a tube at $X_{j}$ such that

$$
\pi_{j 1}^{-1}(x) \subset x+\boldsymbol{R}^{n-j} \quad \text { for } \quad x \in X_{j} .
$$

The existence of $T_{j 1}$ is clear by the definition of tube. We choose $\left|T_{j 1}\right|$ so small that

$$
\pi_{j 1}^{-1}\left(X_{j} \cap V_{j-1}^{\prime}\right) \subset\left|T_{\jmath}^{\prime}\right| \cap V_{\jmath-1}
$$

for some open neighborhood $V_{j-1}^{\prime}$ of $Y_{j-1}$ with $\bar{V}_{j-1}^{\prime}-V_{j-1} \subset \bar{Y}_{j-1}-Y_{j-1}$. Let $\phi$ be a $C^{\infty}$ function on $X_{j}$ such that $\phi=1$ on $X_{j}-V_{j-1}^{\prime}$ and $=0$ on $X_{j} \cap V_{j-1}^{\prime \prime}$ for an open neighborhood $V_{j-1}^{\prime \prime}$ of $Y_{j-1}$ with $\bar{V}_{j-1}^{\prime \prime}-V_{j-1}^{\prime} \subset \bar{Y}_{j-1}-Y_{j-1}$. Put

$$
\pi_{j 2}(x)= \begin{cases}\pi_{j 1}\left(\left(1-\psi^{\circ} \pi_{j 1}(x)\right) \pi_{j}^{\prime}(x)+\psi^{\circ} \pi_{j 1}(x) \pi_{j 1}(x)\right) \\ \pi_{j 1}(x) & \text { for } \quad x \in \pi_{j 1}^{-1}\left(X_{\jmath} \cap V_{j-1}^{\prime}\right) \\ & \text { for } \quad x \in \pi_{j 1}^{-1}\left(X_{\jmath}-V_{j-1}^{\prime}\right) .\end{cases}
$$

As $\left.\pi_{j 2}\right|_{X_{j}}=$ ident, this is well-defined in a neighborhood of $X_{j}$, and we find an open neighborhood $U_{j}^{\prime}$ such that $U_{j}^{\prime} \subset U_{j} \cap\left|T_{j 1}\right|, \bar{U}_{j}^{\prime} \cap \bar{Y}_{j-1} \subset \bar{X}_{j}-X_{j}$, and $\pi_{j 2}: U_{j}^{\prime}$ $\rightarrow X_{j}$ is a $C^{\infty}$ fiber bundle. Let $W_{i}, i=0, \cdots, j-1$, be open neighborhoods of $X_{i}$ such that $\bar{U}_{i}-W_{i} \subset \bar{X}_{i}-X_{i}$. Then by the definition of $\pi_{j 2}$ we can choose $U_{j}^{\prime}$ so small that

$$
\begin{gathered}
\pi_{j 2}^{-1}(x) \subset x+\boldsymbol{R}^{n-m} \quad \text { for } \quad x \in X_{\jmath} \cap\left(W_{m}-W_{0}-\cdots-W_{m-1}\right), \\
m=0, \cdots, j-1 .
\end{gathered}
$$

Now replace $W_{i}$ by $U_{i}, i=0, \cdots, j-1$, and $U_{i}$ by some smaller open neighborhoods 
$U_{i}^{\prime}$ of $X_{i}$ such that $\bar{U}_{i}^{\prime}-U_{i} \subset \bar{X}_{i}-X_{i}$, and regard the induction hypothesis as considered for $U_{\imath}^{\prime}$. Then we can assume by (4.9.3)

$$
\pi_{j 2}^{-1}(x) \subset x+\boldsymbol{R}^{n-m} \quad \text { for } \quad x \in X, \cap\left(U_{m}-U_{0}-\cdots-U_{m-1}\right), m=0, \cdots, j-1 .
$$

It is clear that $\pi_{j 2}=\pi_{j}^{\prime}$ on $\pi_{j 1}^{-1}\left(X_{\jmath} \cap V_{\jmath-1}^{\prime \prime}\right)$. Therefore, considering $\left\{\left.T_{i}^{\prime}\right|_{X_{\imath} \cap r_{j-1}^{\prime \prime}}\right\}$ in place of $\left\{T_{\imath}^{\prime}\right\}$, and shrinking $\left|T_{i}^{\prime}\right|, i=0, \cdots, j$, we may suppose

$$
\begin{aligned}
& \left|T_{\jmath}^{\prime}\right|=\pi_{\jmath 2}^{-1}\left(X_{\jmath} \cap V_{\jmath-1}^{\prime \prime}\right), \\
& \pi_{\jmath 2}=\pi_{\jmath}^{\prime} \text { on }\left|T_{\jmath}^{\prime}\right| \text { and } \\
& \left|T_{2}^{\prime}\right| \subset V_{\jmath-1}^{\prime \prime}, i=0, \cdots, j-1 .
\end{aligned}
$$

Then $\left\{T_{i}^{\prime}\right\}$ continues to be a controlled tube system for $\left\{X_{i} \cap V_{\jmath_{-1}^{\prime \prime}}^{\prime}\right\}$ which satisfies $(4.9 .1,2)$.

Now apply Corollary 4.8 to $\left\{X_{\imath} \cap U_{\jmath}^{\prime}\right\}_{\imath=j, \jmath+1, \ldots}, \pi=\pi_{\jmath 2}: U_{\jmath}^{\prime} \rightarrow X_{j}, U=V_{\jmath-1}^{\prime \prime}$ and a tube system $\left\{T_{i}^{0}\right\}=\left\{\left.T_{i}^{\prime}\right|_{x_{i} \cap U_{j}^{\prime} \cap V_{j-1}^{\prime \prime}}\right\}_{i=j, \ldots}$ for $\left\{X_{i} \cap U_{\jmath}^{\prime} \cap V_{j-1}^{\prime \prime}\right\}_{i=j, \ldots}$. Then we have a controlled tube system $\left\{T_{i 3}=\left(E_{i 3}, \pi_{i 3}, \rho_{i 3}, e_{i 3}\right)\right\}_{i=j, \ldots}$ for $\left\{X_{i} \cap U_{j}^{\prime \prime}\right\}_{i=j, \ldots,} U_{j}^{\prime \prime}$ being an open neighborhood of $X_{j}$ contained in $U_{j}^{\prime}$ such that $\pi_{i 3}=\pi_{j 2}$ on $\left|T_{\jmath 3}\right| \subset U_{j}^{\prime}$ and that

$$
\left.\left.T_{\imath 3}\right|_{x_{\imath} r, U_{j}^{\prime \prime} \wedge V_{j-1}^{\prime \prime \prime}} \equiv T_{\imath}^{\prime}\right|_{x_{\imath} \cap U_{j}^{\prime \prime} \cap V_{j-1}^{\prime \prime \prime}}, i=j, j+1, \cdots
$$

where $V_{j-1}^{\prime \prime \prime}$ is any given open neighborhood of $Y_{j-1}$ so that $\bar{V}_{j-1}^{\prime \prime \prime}-V_{j-1}^{\prime \prime} \subset \bar{Y}_{j-1}-$ $Y_{j-1}$. We remark that the last inclusion implies

$$
\overline{U_{j}^{\prime} \cap V_{j-1}^{\prime \prime \prime}}-V_{j-1}^{\prime \prime} \subset \bar{X}_{j}-X_{j}
$$

because of $\bar{U}_{\jmath}^{\prime} \cap \bar{Y}_{\jmath-1} \subset \bar{X}_{\jmath}-X_{\jmath}$. Shrink $V_{\jmath-1}^{\prime \prime \prime}$ and $U_{\jmath}^{\prime \prime}$, and put $V_{j}=V_{\jmath-1}^{\prime \prime \prime} \cup U_{\jmath}^{\prime \prime}$. Then, since each $T_{\imath}^{\prime}, i=j, j+1, \cdots$, is defined at $X_{i} \cap V_{{ }^{\prime}-1}^{\prime \prime}, T_{\imath 3}$ can be easily extended to a tube at $X_{i} \cap V_{j}$. Here we can not necessarily extend so that iextension $|=| T_{\imath}^{\prime}|\cup| T_{i 3} \mid$. We use the same $T_{i 3}$ for the extension. Shrink $\left|T_{i}^{\prime}\right|, i=0, \cdots, j-1,\left|T_{i 3}\right|, i=j, \cdots$. Then it is easy to see that $\left\{T_{i}^{\prime}\right\}_{i=0, \cdots, j-1} \cup$ $\left\{T_{\imath 3}\right\}_{i=j, \ldots}$ is a controlled tube system for $\left\{X_{i} \cap V_{j}\right\}$ which satisfies $(4.9 .1,2)$, since $\pi_{\jmath 2}=\pi_{\jmath 3}$ satisfies (4.9.2). Hence Lemma 4.9 follows from the induction.

Definition 4.10. Let $\left\{X_{i}\right\}$ be a Whitney stratification of a subset $X$ of $\boldsymbol{R}^{n}$, and $\left\{T_{i}\right\}$ be a controlled tube system for $\left\{X_{i}\right\}$. A vector field $\xi$ on $\left\{\left(X_{2}, T_{\imath}\right)\right\}$ (or $\left.\left\{X_{i}\right\}\right)$ consists of one $C^{\infty}$ rector field $\xi_{i}$ on each $X_{l}$. We call $\xi$ controlled if for any $X_{i}, X_{\imath}$.

$$
\begin{aligned}
& d\left(\left.\pi_{\imath}\right|_{x_{\imath^{\prime}}}\right) \xi_{\imath^{\prime} x}=\xi_{i \pi_{i}(x)}, \quad x \in X_{\iota^{\prime}} \cap U_{i} \\
& d\left(\left.\rho_{i}\right|_{x_{\imath^{\prime}}}\right) \xi_{\imath^{\prime} \infty}=0, \quad x \in X_{\imath^{\prime}} \cap U_{\imath}
\end{aligned}
$$

where $U_{\imath} \subset \mid T_{l}$ ! is some neighborhood of $X_{i}$ in $\boldsymbol{R}^{n}$. We call $\xi$ continuous if for any sequence $\left\{x_{j}\right\}$ of points in $X_{i^{\prime}}$ converging to $x$ of $X_{i},\left\{\xi_{i^{\prime} x_{j}}\right\}$ converges to $\xi_{i x}$ as vectors in $\boldsymbol{R}^{n}$. 
Lemma 4.11. Let $\left\{X_{i}\right\},\left\{T_{i}\right\}$ be the same as the above definition. Assume the index set contains 1 . Let $\xi_{1}^{\prime}$ be a $C^{\infty}$ vector field on $X_{1}$. Then there exists a continuous controlled vector field $\xi=\left\{\xi_{i}\right\}$ on $\left\{\left(X_{i} \cap U,\left.T_{\imath}\right|_{x_{\imath} \cap U}\right)\right\}, U$ being an open neighborhood of $X_{1}$ in $\boldsymbol{R}^{n}$, such that $\xi_{1}=\xi_{1}^{\prime}$.

Proof. Since $\pi_{1}:\left|T_{1}\right| \rightarrow X_{1}, T_{1}=\left(E_{1}, \pi_{1}, \rho_{1}, e_{1}\right)$, is the restriction of a Thom map between $\boldsymbol{R}^{n}$ with respect to $\left\{X_{i}^{\prime}=X_{i} \cap U\right\},\left\{X_{1}\right\}$ for an open neighborhood $U$ of $X_{1}$ (as shown in the proof of Corollary 4.8), the existence of $\xi$ is a consequence of Theorem 3.2, Chapter II, [1], if we do not require $\xi$ to be continuous. For the continuity we need a careful reconsideration of the proof as follows. Using a $C^{\infty}$ partition of unity, we can reduce the problem to the case in which

$$
\begin{aligned}
& \left\{X_{i}\right\}=\left\{X_{1}, X_{2}\right\}, \quad \bar{X}_{2} \cap X_{1} \neq \varnothing, \quad\left|T_{1}\right|=\boldsymbol{R}^{n} . \\
& X_{1}=\left\{\left(x_{1}, \cdots, x_{n}\right) \in \boldsymbol{R}^{n} \mid x_{1}=\cdots=x_{k}=0\right\}=0 \times \boldsymbol{R}^{n-k},
\end{aligned}
$$

and $\pi_{1}: \boldsymbol{R}^{n} \rightarrow X_{1}$ is the projection, and we only have to define $\xi_{2}$ in a neighborhood of $0 \in \boldsymbol{R}^{n}$.

Moreover we can assume

$$
\rho_{1}(x)=x_{1}^{2}+\cdots+x_{k}^{2}, \quad x=\left(x_{1}, \cdots, x_{n}\right) .
$$

Indeed, by the property of $\rho_{1}$, we can suppose

$$
\rho_{1}\left(x_{1}, \cdots, x_{k}, 0, \cdots, 0\right)=x_{1}^{2}+\cdots+x_{k}^{2} .
$$

Then it follows that

$$
\rho_{1}(x)=x_{1}^{2}+\cdots+x_{k}^{2}+\sum_{\substack{j l=1, \cdots, k \\ l=1, \cdots, n}} a_{\imath \jmath l}(x) x_{\imath} x_{\jmath} x_{l}
$$

as germs at 0 for some $C^{\infty}$ function germs $a_{i j l}$. Let us consider the ring of $C^{\infty}$ function germs at 0 in $\boldsymbol{R}^{n}$, let $\mathfrak{m}$ be the maximal ideal, and let $I$ be the ideal generated by $\frac{\partial \rho_{1}}{\partial x_{i}}, i=1, \cdots, k$. Then it is easy to see that $I$ is generated by $x_{1}, \cdots, x_{k}$. Hence $\rho_{1}(x)-x_{1}^{2}-\cdots-x_{k}^{2}$ is an element of $\mathfrak{m} I^{2}$. Then, by a refinement of a result of Tougeron and Mather (Lemma 2, [15]), there exists a local diffeomorphism $\tau$ at 0 in $\boldsymbol{R}^{n}$ such that

$$
\begin{aligned}
& \rho_{1} \circ \tau(x)=x_{1}^{2}+\cdots+x_{k}^{2}, \\
& \tau(x)=\left(\tau_{1}(x), \cdots, \tau_{k}(x), x_{k+1}, \cdots, x_{n}\right) .
\end{aligned}
$$

As $\pi_{1} \circ \tau=\pi_{1}$, considering $\pi_{1}, \rho_{1} \circ \tau$ in place of $\pi_{1}, \rho_{1}$, we may assume (4.11.1).

For any $y \in X_{2}$, the vector $y-\pi_{1}(y)$ is vertical to the tangent space $T_{y} \rho_{1}^{-1}$ $\left(\rho_{1}(y)\right)$ because of (4.11.1). Hence, by the Whitney condition, the tangent spaces $T_{y} X_{2}$ and $T_{y} \rho_{1}^{-1}\left(\rho_{1}(y)\right)$ are transversal if $y$ is near to $X_{1} \cap B, B=\left\{x \in \boldsymbol{R}^{n}|| x \mid \leqq 1\right\}$. We set

$$
V_{y}=T_{y} X_{2} \cap T_{y} \rho_{1}^{-1}\left(\rho_{1}(y)\right)
$$


Now the following statement is sufficient for the proof.

(4.11.2) Let $\left\{x^{i}\right\}$ be a sequence of points in $X_{2}$ converging to $x \in X_{1} \cap B$. If the sequence $\left\{V_{. c}\right\}$ converges to a subspace $L \subset \boldsymbol{R}^{n}$, then $L \supset T_{x} X_{1}=X_{1}$.

The reason is the following. Let $y \in X_{2}$ be a point near to $X_{1} r_{1} B$. (4.11.2) means that we have a basis $\left(v_{1}, \cdots, v_{k^{\prime}}\right)$ of the linear space $V_{y}$ with $k+k^{\prime} \geqq n$ such that

$$
v_{\iota}=\boldsymbol{x}_{k+\imath}+\sum_{\jmath=1}^{n} a_{\iota \jmath} \boldsymbol{x}_{\jmath}, i=1, \cdots, n-k, a_{i \jmath} \in \boldsymbol{R},
$$

where $\boldsymbol{x}_{\jmath}=(0, \cdots, 0, \stackrel{j}{1}, 0, \cdots, 0)$ and if $y$ converges to a point of $X_{1}$ then $\left|a_{\iota \jmath}\right|$ are chosen to converge to 0 . Clearly we can assume $a_{\imath \jmath}=0$ for $j=k+1, \cdots, n$. If

$$
\xi_{1, \pi_{1}(y)}=\sum_{i=1}^{n-k} b_{\imath} \boldsymbol{x}_{k+\imath}, \quad b_{\iota} \in \boldsymbol{R},
$$

then put

$$
\xi_{2 y}=\sum_{i=1}^{n-k} b_{\iota} v_{\imath} .
$$

Then $\xi_{2 y}$ satisfies $(4.10 .1,2)$. On the other hand, by the transversality of $T_{y} X_{2}$ and $T_{y} \rho_{1}^{-1}\left(\rho_{1}(y)\right), \bigcup_{y \in X_{2}} V_{y}$ comes out a $C^{\infty}$ vector subbundle of the tangent bundle of $X_{2}$, and the restriction of $d \pi_{1}$ to $\bigcup_{y \in X_{2}} V_{y}$ is a submersion into the tangent bundle of $X_{1}$. Hence $\xi_{2 y}$ can be extended to a small neighborhood of $y$ holding $(4.10 .1,2)$. Using once more a partition of unity, we easily obtain $\hat{\xi}_{2}$ on $X_{2}$ so that $\left(\xi_{1}, \xi_{2}\right)$ is a continuous controlled vector field on $\left\{\left(X_{1}, T_{1}\right),\left(X_{2}, T_{2}\right)\right\}$.

We want to prove (4.11.2). Without loss of generality, we may suppose that sequence $\left\{\overrightarrow{x^{i} \pi_{1}\left(x^{i}\right)}\right\}$ of lines containing 0 and $x^{i}-\pi_{1}\left(x^{i}\right)$ converges to a line $L_{1} \subset \boldsymbol{R}^{n}$ and that the sequence of tangent spaces $\left\{T_{x i} X_{2}\right\}$ converges to a subspace $L_{2} \subset \boldsymbol{R}^{n}$. Then, by the Whitney condition and Remark 4.6, we have

$$
L_{1} \subset L_{2}, \quad X_{1} \subset L_{2} .
$$

Here we must remark that the Whitney condition is invariant under a diffeomorphism of $\boldsymbol{R}^{n}$. Let $L_{1 x \iota}$ be a unit vector contained in $\overrightarrow{x^{i} \pi_{1}\left(x^{i}\right)}$ and $L_{2, \iota \iota}$ be a unit vector in $T_{x i} X_{2}$ such that $\left\{L_{1 x i}\right\}$ and $\left\{L_{2 x i}\right\}$ converge to $L_{1}$. Then $\left\{L_{1 x i}\right.$ $\left.-L_{2 . x i}\right\}$ converges to 0 , and it follows from (4.11.1) that $L_{1 . c} i$ is vertical to $V_{x i}$ and to $T_{x i} \rho_{1}^{-1}\left(\rho_{1}\left(x^{i}\right)\right)$. Hence if $L_{2 x i}=a_{i} L_{1 x i}+L_{3 x} i, a_{i} \in \boldsymbol{R}, L_{3 x i} \in T_{x i} \rho_{1}^{-1}\left(\rho_{1}\left(x^{i}\right)\right)$, is the orthogonal decomposition, then $\left\{a_{\imath}\right\}$ and $\left\{L_{3 . x}\right\}$ converge to 1 and 0 respectively.

For (4.11.2), we only need to see that for a unit vector $v \subseteq X_{1}$, there exist $i^{,} \in V_{x i}, i=1, \cdots$, such that $\left\{v^{i}\right\}$ converges to $v$. By the inclusion $X_{1} \subset L_{2}$, we have $v^{\prime i} \in T_{x i} X_{2}, i=1, \cdots$, such that $\left\{v^{\prime i}\right\}$ converges to $v^{\prime}$. Since $T_{x i} X_{2}$ is the direct sum of $\boldsymbol{R} L_{2 x^{i}}$ and $V_{x i}$, there exist uniquely $\imath^{i} \in V_{x i}$ and $b_{\iota} \in \boldsymbol{R}$ such that $r^{\prime i}=r^{i}+b_{i} L_{2 x i}$. The sequence $\left\{b_{i}\right\}$ converges to 0 . In fact 


$$
v^{\prime i}=\left(v^{i}+b_{i} L_{3 x i}\right)+a_{i} b_{i} L_{1 x i}
$$

is the orthogonal decomposition of $v^{\prime i}$ to the $T_{x i} \rho_{1}^{-1}\left(\rho_{1}\left(x^{2}\right)\right)$-factor and its normal factor. Since $T_{x^{i}} \rho_{1}^{-1}\left(\rho_{1}\left(x^{i}\right)\right) \supset X_{1}$ as linear spaces, the convergence of $\left\{v^{\prime i}\right\}$ to $v$ implies that of $\left\{v^{i}+b_{i} L_{3 x i}\right\}$ to $v$, which shows that $\left\{a_{i} b_{\imath}\right\}$ converges to 0 . Therefore $b_{i} \rightarrow 0$ as $i \rightarrow \infty$. Hence we prove the convergence of $\left\{v^{i}\right\}$ to $v$ and hence the lemma.

Let $\left\{X_{i}\right\}$ be a Whitney stratification of a subset $X$ of $\boldsymbol{R}^{n}$, and $\xi=\left\{\xi_{i}\right\}$ be a vector field on $\left\{X_{i}\right\}$. Integrating each vector field $\xi_{i}$ on $X_{i}$ we obtain $C^{\infty}$ flows $\Theta_{i}: D_{i} \rightarrow X_{i}$ where $\Theta_{\imath}$ are given by $\Theta_{\imath}(x, 0)=x, \frac{\partial}{\partial t} \Theta_{\imath}(x, t)=\xi_{\Theta_{i}(x, t)}$ and $D_{\imath} \subset$ $X_{i} \times \boldsymbol{R}$ are the maximal domains containing $X_{i} \times 0$. Let $D$ be the union of $D_{\imath}$, and define $\Theta: D \rightarrow X$ by $\left.\Theta\right|_{D_{i}}=\Theta_{i}$.

Definition 4.12. We call $\Theta: D \rightarrow X$ the flow of $\xi$. $\xi$ is called locally integrable if $D$ is open in $X \times R$ and if $\Theta$ is continuous (see Lemma 4.4, Chapter II, [1]).

Lemma 4.13 (Corollary 4.7, Chapter II, [1]). Let $\left\{X_{\imath}\right\}$ be a Whitney stratification of a subset $X$ of $\boldsymbol{R}^{n},\left\{T_{i}\right\}$ be a controlled tube system for $\left\{X_{i}\right\}$, and $\bar{\xi}$ be a controlled vector field on $\left\{\left(X_{2}, T_{2}\right)\right\}$. If $X$ is locally closed, $\xi$ is locally integrable.

Lemma 4.14. Let $\left\{X_{i}\right\}$ be a Whitney stratification of a locally closed subset Y of $\boldsymbol{R}^{n}$, let $f_{1}, f_{2}$ be $C^{\infty}$ functions on $\boldsymbol{R}^{n}$, let $\xi=\left\{\xi_{\imath}\right\}$ be a vector field on $\left\{X_{i}\right\}$, and let $\left\{V_{j}\right\}_{j=0, \cdots, n-1},\left\{V_{j}^{1}\right\}_{j=0, \cdots, n-1}$ be open coverings of $X$ in $\boldsymbol{R}^{n}$ with $\bar{V}_{j}^{1}-V_{j} \subset$ $\bar{X}-X$ for each $j$. Assume for each $i, j$

$$
\tilde{\xi}_{\imath}\left(\left.f_{1}\right|_{x_{\imath}}\right), \xi_{i}\left(\left.f_{2}\right|_{x_{\imath}}\right)>0,
$$

(4.14.2) $x \div \boldsymbol{R}^{l}$ and $X_{\iota}$ are transversal for any $x \in \boldsymbol{R}^{n}$ where $l=\operatorname{codim} X_{i}$, and

$$
\bar{\xi}_{\imath x} \in \boldsymbol{R}^{n-\jmath} \quad \text { for } x \in V_{\jmath} \cap X_{\iota} .
$$

Then there exists a locally integrable vector field $\xi^{1}=\left\{\xi_{l}^{1}\right\}$ on $\left\{X_{i}\right\}$ uhich satisties (4.14.1) and (4.14.3) for $x \in V_{j}^{1} \cap X_{i}$.

Proof. By the same reason as the proof of Lemma 4.9 we assume $\left\{X_{2}\right\}=$ $\left\{X_{\imath}\right\}_{\imath=1,2, \cdots, n}$ and $\operatorname{dim} X_{i}=i$. Here we remark that $X_{0}$ does not exist because of (4.14.1). By (4.14.2, 3), the property $V_{j} \cap X_{i} \neq \varnothing$ implies $j<i$, hence $V_{j} \cap X_{\imath}=\varnothing$ for $j \geqq i$, and for each $i\left\{V_{j}\right\}_{j=0, \cdots, i-1}$ is a covering of $X_{i}$. Let $\left\{V_{j}^{k}\right\}_{j=0, \cdots, n-1}, k=$ $2, \cdots, n$, be open coverings of $X$ such that $\bar{V}_{j}^{n}-V, \subset \bar{X}-X$ and $\bar{V}_{j}^{k-1}-\gamma_{j}^{k} \subset$ $\bar{X}-X, k=2, \cdots, n, j=0, \cdots, n-1$. Then we have $\bar{V}_{\jmath}^{k} \cap X_{i}=\varnothing, k=1, \cdots, n$, if $j \geqq i$. Let $U_{i}$ be an open neighborhood of each $X_{i}$ such that

$$
U_{\imath} \subset \bigcup_{\jmath=0}^{i-1} V_{\jmath}^{1}, \bar{U}_{i} \cap \bar{V}_{\jmath}^{n} \subset \bar{X}_{i}-X_{i} \text { if } j \geqq i
$$


Then, by Lemma 4.9 we have a controlled tube system $\left\{T_{\imath}=\left(E_{i}, \pi_{\imath}, \rho_{\imath}, e_{\imath}\right)\right\}$ for $\left\{X_{i}^{-}\right\}$such that for each $i$

$$
\begin{gathered}
\quad\left|T_{i}\right| \subset U_{i} \\
\pi_{i}^{-1}(x) \subset x+R^{n-k} \quad \text { for } \quad x \in X_{\imath} \cap\left(U_{k}-U_{1}-\cdots-U_{k-1}\right) \\
k=1, \cdots, i .
\end{gathered}
$$

We will show the existence of a controlled vector field $\xi^{1}$ on $\left\{\left(X_{\iota}, T_{\iota}\right)\right\}$ which satisfies (4.14.1) and (4.14.3) for $x \in V_{\jmath}^{1} \cap X_{\imath}$. We prove it by induction. Assume we have already constructed a vector field $\xi^{m+1}=\left\{\xi_{i}^{m+1}\right\}$ on $\left\{\left(X_{i}, T_{i}\right)\right\}$ satisfying (4.14.1) and (4.14.3) for $x \in V_{j}^{m+1} \cap X_{i}$ such that $\left\{\xi_{i}^{m+1}\right\}_{i \geqq m+1}$ is controlled on $\left\{\left(X_{i}, T_{2}\right)\right\}_{i \geqq m+1}$. In the case $m=n-1$, it is trivial, so we want to find $\xi^{m}$. Now Lemma 4.11 implies the existences of an open neighborhood $W$ of $X_{m}$ and a continuous controlled vector field $\xi^{\prime}=\left\{\xi_{\imath}^{\prime}\right\}$ on $\left\{\left(Y_{\imath} \cap \Pi^{*},\left.T_{\imath}\right|_{x_{2},-W}\right)\right\}$ such that $\xi_{m}^{\prime}=\xi_{m}^{m+1}$. Choose $\mathrm{IV}$ so small that

$$
\begin{aligned}
& X_{i} \cap W=\varnothing, \quad i=1, \cdots, m-1, \quad W \subset\left|T_{m}\right|, \\
& W \cap \pi_{m}^{-1}\left(V_{\jmath}^{m} \cap X_{m}\right) \subset V_{\jmath}^{m+1}, \quad j=0, \cdots, m-1, \quad \text { and } \\
& \xi_{i}^{\prime}\left(\left.f_{\jmath}\right|_{x_{\imath} \sim W}\right)>0 \quad \text { for } \quad i=m, \cdots, j=1,2 .
\end{aligned}
$$

The last inequality follows from the continuity of $\xi^{\prime}$.

It is easy to find a positive $C^{\infty}$ function $\theta$ on $X_{m}$ such that $W^{\prime}=\{x \in W \mid \theta$ 。 $\left.\pi_{m}(x) \cdot \rho_{m}(x)<1\right\}$ satisfies $\bar{W}^{\prime}-W \subset \bar{X}_{m}-X_{m}$. Let $\phi$ be a $C^{\infty}$ function on $\boldsymbol{R}$ such that $\phi=0$ on $[1, \infty),=1$ on $(-\infty, 1 / 2]$ and $0<\phi<1$ on $(1 / 2,1)$. Put

$$
\Psi(x)= \begin{cases}\phi\left(\theta \circ \pi_{m}(x) \cdot \rho_{m}(x)\right) & \text { for } x \in W^{\top} \\ 0 & \text { for } x \in \boldsymbol{R}^{n}-W-\left(\bar{X}_{m}-X_{m}\right) .\end{cases}
$$

Then $\Psi$ is a $C^{\infty}$ function on $\boldsymbol{R}^{n}-\left(\bar{X}_{m}-X_{m}\right)$ such that $\Psi=1$ in a neighborhood of $X_{m}$, $=0$ outside $W, 0 \leqq \Psi \leqq 1$ and

$$
\Psi(x)=\Psi \circ \pi_{\imath}(x) \quad \text { for } \quad x \in\left|T_{i}\right|, i \geqq m+1
$$

by $(4.2 .1,2)$. Here we have to shrink $\left|T_{i}\right|, i \geqq m+1$, in order to assure that if $x \in W^{\prime} \cap\left|T_{i}\right|$, then $\pi_{i}(x) \in\left|T_{m}\right|$ and conversely that if $x \in\left|T_{i}\right|, \pi_{i}(x) \in W^{\prime}$, then $x \equiv\left|T_{m}\right|$. Put

$$
\bar{\xi}_{i}^{m}= \begin{cases}\Psi \xi_{i}^{\prime}+(1-\Psi) \xi_{i}^{m+1} & \text { for } i \geqq m \\ \xi_{i}^{m+1} & \text { for } i<m .\end{cases}
$$

Then $\xi^{m}=\left\{\xi_{i}^{m}\right\}$ is a vector field on $\left\{\left(X_{i}, T_{i}\right)\right\}$ satisfying (4.14.1) and (4.14.3) for $x \leqq V_{j}^{m} \cap X_{i}$ and $\left\{\xi_{i}^{m}\right\}_{i \geqq m}$ is controlled on $\left\{\left(X_{i}, T_{i}\right)\right\}_{i \geqq m}$ as follows.

Indeed (4.14.1) is clear. For (4.14.3) it is sufficient to see

$$
\xi_{i, x}^{\prime} \in \boldsymbol{R}^{n-j} \quad \text { for } \quad x \in V_{\jmath}^{m} \cap X_{i} \cap W
$$


because of the assumption on $\xi^{m+1}$. By the property $X_{i} \cap W=\varnothing, i=1, \cdots, m-1$, $X_{i}$ in (4.14.8) is of dimension $\geqq m$. Since $\xi^{\prime}$ is controlled, we see

$$
\begin{aligned}
\xi_{\iota x}^{\prime} \in \boldsymbol{R}^{n-\min (j, k)} \quad \text { for } \quad x \in W \text { with } \pi_{m}(x) \in X_{m} \cap \\
\left(\bar{U}_{k}-\bar{U}_{1}-\cdots-\bar{U}_{k-1}\right) \cap V_{J}^{m+1}, 1 \leqq k \leqq m, 0 \leqq j \leqq m-1
\end{aligned}
$$

by (4.10.1), (4.14.6), the assumption on $\xi_{m}^{\prime}=\xi_{m}^{m+1}$ and by easy basic calculations. For any $x \in W$ with $\pi_{m}(x) \in X_{m} \cap\left(\bar{U}_{k}-\bar{U}_{1}-\cdots-\bar{U}_{k-1}\right) \cap V_{\jmath}^{m+1}$, let $j_{x}$ denote the maximum of such $j$. Since $\bar{U}_{k} \cap V_{\jmath_{x}}^{m+1} \subset \bar{X}_{k}-X_{k}$ for $k \leqq j_{x}$ (4.14.4), and since $1 \leqq k \leqq m$, the property $X_{m} \cap \bar{U}_{k} \cap V_{J_{x}}^{m+1} \neq \varnothing$ means that $k>j_{x}$. Hence we have by (4.14.9)

$$
\xi_{i x}^{\prime} \in \boldsymbol{R}^{n-j} \quad \text { for } \quad x \in W \text { with } \pi_{m}(x) \in V_{j}^{m+1} \cap X_{m} .
$$

Therefore, shrinking $\left|T_{m}\right|$ and hence $W, W^{\prime}$, we obtain (4.14.8).

It rests to see that the vector field $\left\{\xi_{i}^{m}\right\}_{i \geqq m}$ on $\left\{\left(X_{i}, T_{i}\right)\right\}_{i \geqq m}$ is controlled. For any $X_{i}, X_{i^{\prime}}, i^{\prime}>i \geqq m$ and $x \in X_{i^{\prime}}$ near to $X_{i}$, we have if $x \in W$,

$$
\begin{aligned}
& d\left(\left.\pi_{i}\right|_{x_{i^{\prime}}}\right) \xi_{i^{\prime} x}^{m}=\Psi(x) d\left(\left.\pi_{i}\right|_{x_{\imath^{\prime}}}\right) \xi_{\imath^{\prime} x}^{\prime}+(1-\Psi(x)) d\left(\left.\pi_{\imath}\right|_{x_{\imath^{\prime}}}\right) \xi_{\imath^{\prime} x}^{m+1} \\
= & \Psi \circ \pi_{\imath}(x) d\left(\left.\pi_{i}\right|_{x_{\imath^{\prime}}}\right) \xi_{i^{\prime} x}^{\prime}+\left(1-\Psi \circ \pi_{i}(x)\right) d\left(\left.\pi_{i}\right|_{x_{\imath^{\prime}}}\right) \xi_{\imath^{\prime} x}^{m+1} \text {, by }(4.14 .7), \\
= & \Psi \circ \pi_{\imath}(x) \xi_{i \pi_{i}(x)}^{\prime}+\left(1-\Psi \circ \pi_{i}(x)\right) \xi_{i \pi_{\imath}(x)}^{m+1}, \text { by the controlledness } \\
= & \xi_{\imath \pi_{i}(x)}^{m}, \quad \text { of } \xi^{\prime} \text { and }\left\{\xi_{\imath}^{m+1}\right\}_{\imath=m+1},
\end{aligned}
$$

and if $x \notin W$, by (4.14.7) and the controlledness of $\left\{\xi_{i}^{m+1}\right\}_{i \geqq m+1}$

$$
d\left(\left.\pi_{i}\right|_{X_{i^{\prime}}}\right) \xi_{i^{\prime} x}^{m}=d\left(\left.\pi_{i}\right|_{X_{\imath^{\prime}}}\right) \xi_{i^{\prime} x}^{m+1}=\xi_{\imath \pi_{i}(x)}^{m+1}=\xi_{i \pi_{i}(x)}^{m} .
$$

Hence (4.10.1) holds true. (4.10.2) is shown in the same way by the controlledness of $\xi^{\prime}$ and $\left\{\xi_{\imath}^{m+1}\right\}_{i \geqq m+1}$. We omit the proof. Hence, by induction we have a controlled vector field $\xi^{1}$ on $\left\{\left(X_{i}, T_{i}\right)\right\}$ which satisfies (4.14.1) and (4.14.3) for $x \in V^{1} \cap X_{i}$, which, together with Lemma 4.13, proves Lemma 4.14.

\section{§5. Topological Equivalence of Subanalytic Functions}

In this section we prove the following.

Proposition 5.1. Let $X$ be a closed subanalytic subset of $\boldsymbol{R}^{n}$. Let $f_{1}, f_{2}$ be subanalytic functions on $X$ such that for each point $x \in X$, both $f_{1}(x)$ and $f_{\mathrm{2}}(x)$ have the same sign. Put $Z=f_{1}^{-1}(0)$. Then there exist neighborhoods $W_{1}, W_{2}$ of $Z$ in $X$ and a homeomorphism $\tau: W_{1} \rightarrow W_{2}$ such that $f_{2}^{\circ} \tau=f_{1}$ on $W_{1}$ and $\left.\tau\right|_{Z}=$ ident.

The case of analytic function germs is Theorem 4.3 in [16]. There I gave a sketch of proof, whose idea is the main tool also in the proof below. Two 
functions $f_{1}, f_{2}$ on a manifold $M$ is called topologically equivalent if there exists a homeomorphism $\tau$ of $M$ such that $f_{1}{ }^{\circ} \tau=f_{2}$. Another version of Proposition 5.1 is Corollary 4.4, [16], which should be amended to the following.

Proposition 5.2. Let $f_{1}, f_{2}$ be analytic functions on a compact analytic manifold M. Put

$$
S=\left\{x \in M \mid a_{1} d f_{1 x}+a_{2} d f_{2.2}=0 \text { for some } a_{1}, a_{2} \geqq 0 \text { with } a_{1}+a_{2}>0\right\} .
$$

Assume $f_{1}^{-1} f_{1}(S)=f_{2}^{-1} f_{2}(S)$ and $f_{1}=f_{2}$ on $f_{1}^{-1} f_{1}(S)$. Then $f_{1}$ and $f_{2}$ are topologically equivalent, and the homeomorphism of equivalence can be the identity on $f_{1}^{-1} f_{1}(S)$ and analytic outside it.

The proof of Proposition 5.1 consists of 5 steps, (5.3), $\cdots,(5.7)$.

(5.3) Let $\left\{X_{i}\right\}_{i=1, \ldots}$ be a subanalytic stratification of $X$ compatible with $Z$ (2.11). We can reduce the problem to the case in which $(5.3 .1, \cdots, 5)$ below hold true.

(5.3.1) $f_{1}, f_{2}$ are restrictions to $X$ of analytic functions on $\boldsymbol{R}^{n}$.

(5.3.2) $\quad\left\{X_{\imath}\right\}$ is a Whitney stratification of $X$.

(5.3.3) There exist compact analytic manifolds $M_{l}, i=1, \cdots$, subanalytic open subsets $M_{i}^{\prime}$ of $M_{i}$ and analytic maps $\phi_{\imath}: M_{i} \rightarrow \boldsymbol{R}^{n}$ such that $\left.\phi_{\imath}\right|_{M_{\imath}^{\prime}}$ are diffeomorphisms onto $X_{i}$.

Put

and

$$
\boldsymbol{R}^{k}=\left\{\left(x_{1}, \cdots, x_{n}\right) \in \boldsymbol{R}^{n} \mid x_{k+1}=\cdots=x_{n}=0\right\}, \quad 1 \leqq k \leqq n,
$$

$$
x+\boldsymbol{R}^{k}=\left\{x+y \mid y \in \boldsymbol{R}^{k}\right\} \quad \text { for } \quad x \in \boldsymbol{R}^{n} .
$$

(5.3.4) For each $i, k$ and $x \in \boldsymbol{R}^{n}, X_{\imath}$ and $x+\boldsymbol{R}^{k}$ are transversal if $\operatorname{dim} X_{\imath}+k \geqq n$, and $X_{\imath} \cap\left(x+\boldsymbol{R}^{k}\right)$ is empty or of dimension 0 if $\operatorname{dim} X_{i}+k<n$.

Let $N \subset X$ be a subanalytic analytic submanifold in $\boldsymbol{R}^{n}$, and let $1 \leqq k \leqq n$ with $\operatorname{dim} N+k>n$. Assume that for any $x \in \boldsymbol{R}^{n}, N$ and $x+\boldsymbol{R}^{k}$ are transversal. Put

$$
\begin{aligned}
S(N, k)= & \left\{x \in N \mid a_{1} d\left(\left.f_{1}\right|_{x \cap x+R k}\right)_{x}+a_{2} d\left(\left.f_{2}\right|_{x \cap x+R k}\right)_{x}=0\right. \\
& \text { for some } \left.a_{1}, a_{2} \geqq 0 \text { with } a_{1}+a_{2}>0\right\} .
\end{aligned}
$$

(5.3.5) For each $X_{\imath} \subset Z, X_{\iota^{\prime}} \not \subset Z$ and $1 \leqq k \leqq n$ with $\operatorname{dim} X_{\imath}+k \geqq n$ and $\bar{X}_{\iota^{\prime}} \supset \mathrm{Y}_{\iota}$, $S\left(X_{l^{\prime}}, k\right)$ is empty in a neighborhood of $X_{\imath}$.

The reduction to (5.3.1) is trivial if we consider the graph of $\left(f_{1}, f_{2}\right)$ in place of $X$. Hence we assume (5.3.1). Before beginning the proof, we remark some facts.

Let $N \subset \boldsymbol{R}^{n}$ be a subanalytic analytic manifold, $M$ be a compact analytic manifold, $M^{\prime}$ be a subanalytic open subset of $M$, and $\psi: M \rightarrow \mathbb{R}^{n}$ be an analytic 
map such that $\left.\phi\right|_{M^{\prime}}$ is a diffeomorphism onto $N$ and that $\phi(M) \subset X$. Let $1 \leqq k$ $\leqq n$ with $\operatorname{dim} N+k>n$. Assume that for any $x \in \boldsymbol{R}^{n}, N$ and $x+\boldsymbol{R}^{k}$ are transversal. Then

(5.3.6) $S(N, k)$ is closed in $N$ and subanalytic.

Proof of (5.3.6). The closedness is clear. For the subanalyticness, consider

$$
V=\left\{v \in T_{x} M \mid x \in M, d \psi_{x} v \in \boldsymbol{R}^{k}\right\} .
$$

Then $V$ is an analytic subset of the tangent bundle $T M$ of $M$ since $d \psi: T M \rightarrow$ $T \boldsymbol{R}^{n}$ is analytic. Put

$$
V^{\prime}=\{v \in V|| v \mid=1\}
$$

for some analytic Riemannian metric on $T M$,

$$
V^{\prime \prime}=\left\{v \in T_{x} M \mid x \in M, d\left(f_{1} \circ \phi\right)_{x} v \cdot d\left(f_{2}^{\circ} \circ\right)_{x} v>0\right\} \text { 。 }
$$

Then $V^{\prime}$ is an analytic subset of $T M$, and $V^{\prime \prime}$ is a well-defined semi-analytic subset of $T M$ by (5.3.1). It is easy to see that

$$
N-S(N, k)=p \circ d \phi\left(V^{\prime} \cap V^{\prime \prime} \cap T M^{\prime}\right)
$$

where $p: T \boldsymbol{R}^{n} \rightarrow \boldsymbol{R}^{n}$ be the projection to the base space. As the closure of $V^{\prime} \cap V^{\prime \prime} \cap T M^{\prime}$ in $T M$ is compact, and as $p \circ d \psi: T M \rightarrow \boldsymbol{R}^{n}$ is analytic, it follows from (2.3) and (2.6) that $p \circ d \psi\left(V^{\prime} \cap V^{\prime \prime} \cap T M^{\prime}\right)$ is subanalytic. Hence (2.1.3) implies that $S(N, k)$ is subanalytic.

(5.3.7) Let $N \subset X-Z$ be a subanalytic analytic submanifold in $\boldsymbol{R}^{n}$. Assume $S(N, n)$ is subanalytic. Then $S(N, n)$ is empty in a neighborhood of $Z$.

Proof of (5.3.7). Assume $\overline{S(N, n)} \cap Z \neq \varnothing$. Apply Proposition 3.1 to $S(N, n)$. Then we have a 1-dimensional subanalytic analytic submanifold $N^{\prime} \subset S(N, n)$ so that $\bar{N}^{\prime} \cap Z \neq \varnothing$. Since $n+\operatorname{dim} N^{\prime}>n, S\left(N^{\prime}, n\right)$ is well-defined. This is the reason why we assumed $k=n$. Now it is easy to see that

$$
S\left(N^{\prime}, n\right)=S(N, n) \cap N^{\prime}=N^{\prime} \text {. }
$$

Applying (2.4) to $N^{\prime}$, we obtain a compact 1-dimensional analytic manifold $M$ and an analytic map $\phi: M \rightarrow \mathbb{R}^{n}$ such that $\phi(M)=\bar{N}^{\prime}$. We remark that $f_{1} \circ \phi(x)$, $f_{2}^{\circ} \phi(x)$ have the same sign for each point $x \in M$, that

$$
f_{1} \circ \psi\left(x_{0}\right)=f_{2} \circ \psi\left(x_{0}\right)=0
$$

for some $x_{0} \in M$ and that

$$
a_{1} d\left(f_{1} \circ \psi\right)_{x}+a_{2} d\left(f_{2} \circ \psi\right)_{x}=0
$$

for each $x \in M$ and some $a_{1}, a_{2} \geqq 0$ with $a_{1}+a_{2}>0$. The third property implies that if one of $f_{i} \circ \mathrm{\psi}$ is monotone increasing on a subset of $M$ diffeomorphic to an interval, then the other is monotone decreasing. This contradicts the first 
and second properties. Hence $\overline{S(N, n)} \cap Z=\varnothing$, which proves (5.3.7).

We will prove (5.3) by induction. Let $0 \leqq l \leqq n$ be an integer. If $l=n$, the next statement coincides with (5.3).

$(5.3)_{l}$ We can reduce the problem to the case in which (5.3.3) for codim $X_{\iota}$ $\leqq l,(5.3 .2)_{l}$ below and $(5.3 .4,5)$ for $k \leqq l$ hold true.

$(5.3 .2)_{l} \quad\left\{X_{i} \mid \operatorname{codim} X_{i} \leqq l\right\}$ is a Whitney stratification of the union of such $X_{\imath}$ 's.

Proof of $(5.3)_{l}$ If $l>0$, assume $(5.3)_{l-1}$. Let $\left\{X_{i}^{\prime}\right\}$ be a subanalytic stratification of $X$ compatible with $Z$ such that (5.3.3) for $\operatorname{codim} X_{\imath}^{\prime}<l,(5.3 .2)_{l-1}$ and $(5.3 .4,5)$ for $k<l$ hold true. Here $X_{i}$ in $(5.3 .3,4,5)$ and $(5.3 .2)_{l-1}$ are replaced by $X_{i}^{\prime}$. Let $I_{1}, I_{2}$ be the index subsets of all $i$ with $\operatorname{codim} X_{2}^{\prime}=l, \geqq l$ respectively, and $Y$ be the union of $X_{i}^{\prime}, i \in I_{2}$.

At first we want to change the $x_{l}$-axis fixing the $x_{1}, \cdots, x_{l-1}$-axes so that $Y \cap\left(x+\boldsymbol{R}^{l}\right)$ is empty or of dimension 0 for any $x \in \boldsymbol{R}^{n}$. This condition is equivalent to that $X_{i}^{\prime} \cap\left(x+\boldsymbol{R}^{l}\right)$ is a finite set for any $i \in I_{2}$ and $x \in \boldsymbol{R}^{n}$, since a bounded subanalytic set of dimension 0 consists of finite points. Let

$$
r_{l-1}: \boldsymbol{R}^{n} \rightarrow \boldsymbol{R}^{l-1 \perp}=\left\{x \in \boldsymbol{R}^{n} \mid x=\left(0, \cdots, 0, x_{l}, \cdots, x_{n}\right)\right\}
$$

be the projection. Then, since $r_{l-1}\left(X_{i}^{\prime}\right)$ is a subanalytic set of dimension $\leqq n-l$ for any $i \in I_{2}$, we can apply Lemma $3.2^{\prime}$ to $r_{l-1}\left(X_{\imath}^{\prime}\right)$. Hence there exists a $1-$ dimensional linear subspace $L$ of $\boldsymbol{R}^{l-1 \perp}$ such that for any $a \in \mathbb{R}^{l-1 \perp}, a+L$ is nonsingular for each $r_{l-1}\left(X_{i}^{\prime}\right), i \in I_{2}$. This means that $(a+L) \cap r_{l-1}\left(X_{\imath}^{\prime}\right)$ is a finite set for each $i \in I_{2}$. Changing the $x_{l}$-axis, we assume that $L$ is the $x_{l}$-axis. Let $x \in \boldsymbol{R}^{n}, i \in I_{2}$. Put $a=r_{l-1}(x)$. Let $(a+L) \cap r_{l-1}\left(X_{i}^{\prime}\right)$ consists of $a_{1}, \cdots, a_{l} \in$ $\boldsymbol{R}^{l-1 \perp} \subset \boldsymbol{R}^{n}$, and let $X_{\imath}^{\prime} \cap\left(a_{j}+\boldsymbol{R}^{l-1}\right)$ consist of at most $l^{\prime \prime}$ points for any $j(5.3)_{l-1}$. Then we have

$$
\begin{aligned}
& \sharp\left(X_{\imath}^{\prime} \cap\left(x+\boldsymbol{R}^{l}\right)\right)=\sharp\left(X_{i}^{\prime} \cap\left(x+\mathbb{R}^{l-1}+L\right)\right) \\
& \leqq \max _{j} \sharp\left(X_{\imath}^{\prime} \cap\left(a_{\jmath}+\boldsymbol{R}^{l-1}\right)\right) \cdot \sharp\left((a+L) \cap r_{l-1}\left(X_{\imath}^{\prime}\right)\right)=l^{\prime} l^{\prime \prime} .
\end{aligned}
$$

Hence $X_{\imath}^{\prime} \cap\left(x+\mathbb{R}^{l}\right)$ is a finite set.

Let $r_{l}: \mathbb{R}^{n} \rightarrow \mathbb{R}^{l \perp}=\left\{x=\left(0, \cdots, 0, x_{l+1}, \cdots, x_{n}\right)\right\}$ be the projection. Since $X_{\imath}^{\prime} \cap\left(x+\mathbb{R}^{l}\right)$ is a finite set for any $i \in I_{1}$ and $x \in \mathbb{R}^{n}, d\left(\left.r_{l}\right|_{x_{2}^{\prime}}\right)$ has the maximal rank $n-l$. By (2.4) there exist compact analytic manifolds $M_{i}$, $i \in I_{1}$, of dimension $n-l$ and analytic maps $\phi_{i}: M_{i} \rightarrow \mathbb{R}^{n}$ such that $\dot{\phi}_{\imath}\left(M_{i}\right)=\bar{X}_{i}^{\prime}$. Let $X_{\imath}^{2}$ be the images under $\phi_{i}$ of the sets of critical points of $r_{l}{ }^{\circ} \phi_{i}$, and $X_{2}^{3} \subset X_{i}^{\prime}$ be subanalytic subsets closed in $X_{i}^{\prime}$ of codimension $>l$ in $\mathbb{R}^{n}$ such that $\left(X_{\jmath}^{\prime}, X_{2}^{\prime}-X_{\imath}^{3}\right)$ satisfies the Whitney condition for any $j \in I_{2}$ (2.12). Here we put $X_{\imath}^{3}=\varnothing$ if $l=0$. Then $Y_{\imath}^{2}$ are of codimension $>l$, and $X_{i}^{\prime}-X_{\imath}^{2}$ and $x+R^{l}$ are transversal for ar $x=$ $\mathbb{R}^{n}$ since $r_{l}: X_{i}^{\prime}-X_{\imath}^{2} \rightarrow \mathbb{R}^{l \perp}$ are immersions. 
For each $i \in I_{1}$ with $X_{\imath}^{\prime} \subset Z$, put

$$
X_{\imath}^{4}=X_{i}^{\prime} \cap \bigcup_{j \notin I_{2}} \bar{S}\left(X_{j}^{\prime}, l\right) .
$$

Here $\bar{S}$ means the closure of $S$ in $\boldsymbol{R}^{n}$, and $S$ is well-defined because the transversality of $X_{j}^{\prime}$ and $x+\boldsymbol{R}^{l-1}$ implies that of $X_{j}^{\prime}$ and $x+\boldsymbol{R}^{l}$. By (5.3.6), $S\left(X_{j}^{\prime}, l\right)$ is closed in $X_{j}^{\prime}$ and subanalytic. Hence (2.1.2) imply that $X_{\imath}^{4}$ is subanalytic.

Moreover $X_{2}^{4}$ is of codimension $>l$ in $\boldsymbol{R}^{n}$. We will prove this by reduction to absurdity. Assume codim $X_{\imath}^{4}=l$. Then (2.2) implies that $\bigcup_{\jmath \notin I_{2}} S\left(X_{\jmath}^{\prime}, l\right)$ is codimension $<l$ as germ at $X_{\imath}^{\prime}$. Apply Proposition 3.1 to $\left\{S\left(X_{j}^{\prime}, l\right)\right\}_{j \notin I_{2}}, X_{\imath}^{4}$. There exist then a triangulation $K$ of $\boldsymbol{R}^{n}$ and a subanalytic homeomorphism $\tau$ of $\boldsymbol{R}^{n}$ such that two properties corresponding to $(3.1 .3,4)$ are satisfied. Hence we have a simplex $\sigma \in K$ of codimension $<l$ so that $\tau\left({ }^{\circ}\right)$ is a subanalytic analytic manifold contained in $S\left(X_{j}^{\prime}, l\right)$ for some $j \notin I_{2}$ and that an open face $\delta^{\prime}$ of $\sigma$ of codimension $l$ is analytically imbedded by $\tau$ into $X_{\imath}^{4}$. By (2.12) there is an inner point $x$ of $\tau\left(\sigma^{\prime}\right)$ such that $\left(\tau\left({ }^{\circ}\right), \tau\left(\dot{\sigma}^{\prime}\right) \cap\right.$ a neighborhood of $\left.x\right)$ satisfies the Whitney condition. Choosing moreover $x \notin X_{\imath}^{2}$, we assume $\tau\left(\dot{\sigma}^{\prime}\right)$ and $x+\boldsymbol{R}^{l}$ are transversal at $x$. Then it follows from the definition of the Whitney condition that $\tau(\dot{\sigma})$ and $x+$ $\boldsymbol{R}^{l}$ are transversal in a neighborhood of $x$, and hence $\tau(\dot{\sigma}) \cap\left(x+\boldsymbol{R}^{l}\right)$ is a subanalytic analytic submanifold of $\boldsymbol{R}^{n}$ in a neighborhood of $x$ whose closure contains $x$. We see easily that

$$
S\left(\tau(\stackrel{\circ}{)}, l)=S\left(X_{j}^{\prime}, l\right) \cap \tau(\stackrel{\circ}{\sigma})=\tau(\stackrel{\circ}{\sigma})\right.
$$

in a neighborhood of $x$. Hence it follows that

$$
a_{1} d\left(\left.f_{1}\right|_{\tau(\hat{\sigma}) \cap(x+R l)}\right)_{y}+a_{2} d\left(\left.f_{2}\right|_{\tau(\hat{\sigma}) \cap(c+R l)}\right)_{y}=0
$$

in a neighborhood of $x$ for each $y \in \tau(\stackrel{\sigma}{\sigma}) \cap\left(x+\boldsymbol{R}^{l}\right)$ and some $a_{1}, a_{2} \geqq 0$ with $a_{1}+$ $a_{2}>0$. These imply that

$$
S\left(\tau(\hat{\sigma}) \cap\left(x+\boldsymbol{R}^{l}\right), n\right)=\tau(\hat{\sigma}) \cap\left(x+\boldsymbol{R}^{l}\right) \text { in a neighborhood of } x,
$$

$$
\overline{\tau(\dot{\sigma}) \cap\left(x+\boldsymbol{R}^{l}\right)} \ni x .
$$

These contradict (5.3.7), hence $\operatorname{codim} X_{l}^{4}>l$.

If $X_{i}^{\prime} \not \subset Z$, put $X_{\imath}^{4}=\varnothing$. Let $\left\{X_{t}\right\}^{l}$ be a subanalytic stratification of $Y$ compatible with $\left\{X_{\imath}^{\prime}, X_{i}^{\prime} \cap X_{\imath}^{2}, X_{i}^{3}, X_{\imath}^{4}\right\}_{i \in I_{1}} \cup\{Z\}$. By Proposition 3.1 we may assume that each $X_{i}$ is simply connected. Put

$$
\left\{X_{\imath}\right\}=\left\{X_{i \notin I_{2}}^{\prime} \cup\left\{X_{\imath}\right\}^{l}\right. \text { 。 }
$$

Then $\left\{X_{i}\right\}$ is a subanalytic stratification of $X$ compatible with $Z$ and satisfying (5.3.3) for $\operatorname{codim} X_{i} \leqq l,(5.3 .2)_{l}$ and $(5.3 .4,5)$ for $k \leqq l$.

Indeed, for any $X_{i}$ with codim $X_{i}=l$ we have $X_{i} \subset X_{\jmath}^{\prime}-X_{j}^{2}-X_{j}^{3}-X_{j}^{4}$ for some $j \in I_{1}$. It is easy to see that for any connected component $A$ of $\phi_{j}^{-1}\left(X_{j}^{\prime}-X_{j}^{2}\right.$ $\left.-X_{j}^{3}-X_{j}^{4}\right)$ 


$$
\psi_{\jmath}: A \longrightarrow X_{\jmath}^{\prime}-X_{\jmath}^{2}-X_{\jmath}^{3}-X_{\jmath}^{4}
$$

is an analytic covering because of the compactness of $M_{\jmath}$. Hence if we let $M_{2}^{\prime}$ be any connected component of $\psi_{j}^{-1} X_{i}$, then $M_{i}^{\prime}$ is a subanalylic open subset of $M_{i}$ by (2.2), (2.5.1), and $\phi_{j}: M_{\imath}^{\prime} \rightarrow X_{i}$ is an analytic diffeomorphism since $X_{i}$ is simply connected. Hence (5.3.3) holds true for codim $X_{i}=l$ and obviously for codim $X_{i}<l$. Now, since each $X_{\imath}$ of codimension $l$ is contained in $Y_{j}^{\prime}-X_{3}^{3}$ for some $j \in I_{1},(5.3 .2)_{l}$ is clear.

Consider (5.3.4) for $k \leqq l$. We have five cases; (i) codim $\AA_{i}<l$ and $k<l$. (ii) $\operatorname{codim} X_{\imath}<l$ and $k=l$, (iii) $\operatorname{codim} X_{\imath}=l$ and $k=l$, (iv) $\operatorname{codim} I_{\iota} \geqq l$ and $k<l$ and (v) $\operatorname{codim} X_{i}>l$ and $k=l$. In the case of (i), (5.3.4) is contained in the assumption. If (ii), we have $\operatorname{dim} X_{i}+k>n$. Hence, by the assumption, $X_{i}$ and $x+\boldsymbol{R}^{k-l}$ are transversal for any $x \in \boldsymbol{R}^{n}$, which implies also the transversality of $X_{\imath}$ and $x+\boldsymbol{R}^{k}$. (5.3.4) in the case of (iii) follows from the fact already seen that $X_{\jmath}^{\prime}-Y_{j}^{2}$ and $x+\boldsymbol{R}^{l}$ are transversal for any $x \in \boldsymbol{R}^{n}, j \in I_{1}$. In the case of (iv) we have $X_{3}^{\prime}, j \in I_{2}$, so that $X_{i} \sqsubset X_{\jmath}^{\prime}$. Hence it is sufficient to see that $X_{\jmath}^{\prime} \frown$ $\left(x+\boldsymbol{R}^{k}\right)$ is empty or of dimension 0 . But it is a part of the assumption because of $k<l, \operatorname{dim} X_{2}^{\prime}+k<n$. We have chosen the $x_{l}$-axis so that $I^{-}-\left(x-R^{l}\right)$ is empty or of dimension 0 for any $x \in \boldsymbol{R}^{n}$. That proves the case of $(\mathrm{v})$.

For (5.3.5), let $X_{\imath} \subset Z, X_{i^{\prime}} \not \subset Z, 1 \leqq k \leqq l$ such that $\operatorname{dim} X_{\imath}+k \geqq n, \overline{\mathrm{I}}_{\imath^{\prime}} \supset X_{2}$. If $S\left(X_{\imath^{\prime}}, k\right)$ is empty in a neighborhood of $X_{\imath}$, then so is $S\left(X_{\imath^{\prime}}, k-1\right)$ because of the inclusion

$$
S\left(X_{\iota}^{\prime}, k\right) \supset S\left(X_{\iota}^{\prime}, k+1\right) .
$$

Hence we only have to prove the case $\operatorname{dim} X_{\imath}+k=n$. There are then two cases $k<l$ or $k=l$. If $k<l$, then $X_{\imath}=X_{\jmath}^{\prime}, X_{i^{\prime}}=X_{\jmath^{\prime}}^{\prime}$ for some $i, i^{\prime}$. Hence, by the assumption, $S\left(X_{l^{\prime}}, k\right)$ is empty in a neighborhood of $X_{\imath}$. If $k=l$, the same statement follows from the facts that $X_{i}$ is contained in $X_{j}^{\prime}-I_{\text {; }}$ for some $j$ and that $X_{\imath^{\prime}}$ coincides with some $X_{\jmath^{\prime}}^{\prime}$.

Thus we have proved $(5.3)_{0}$ and $(5.3)_{l}, l>0$, assuming $(5.3)_{l-1}$. Hence $(5.3)_{n}$ $=(5.3)$ follows.

(5.4) Let $\left\{X_{\imath}\right\}$ be the stratification which appeared in (5.3), $I_{\imath}$ be a stratum with $X_{\imath}, \subset Z$, and $l$ be an integer bigger than $\operatorname{codim} X_{\imath}$. Let $I_{\text {" }}$ denote the union of $X_{\jmath}$ 's such that $X_{\jmath} \subset Z \cap \bar{X}_{\imath}$ and $\operatorname{codim} X_{\jmath}=l$. Then there exists an analytic vector field $\xi_{i l}$ on the intersection $U_{\imath l}$ of $X_{\imath}$ and an open neighborhood of $Y_{i l}$ in $X_{\imath} \cup Y_{\imath l}$ such that

(5.4.1) for any $x \in U_{\imath l}, \xi_{\imath l x}$ is contained in $R^{l}$, and

(5.4.2) $\xi_{l l} f_{k}, k=1,2$, are positive on $U_{l l}$.

Here $\tilde{s}_{i l} f_{k}$ are well-defined because of $(5.3 .1)$.

Proof. Put

$$
U_{\imath l}=X_{i}-S\left(X_{\imath}, l\right),
$$




$$
\begin{aligned}
& \imath_{k \imath l x}=d\left(\left.f_{k}\right|_{x_{\imath} \cap(x+R l}\right)_{x}, \quad k=1,2, x \in U_{\imath \iota}, \\
& \tilde{\xi}_{i l x}=\left|v_{2 i l x}\right| v_{1 i l x}+\left|v_{1 i l x}\right| v_{2 \imath l x}, x \in U_{\imath l} .
\end{aligned}
$$

Then, by $\left(5.3 .4, v_{l i \imath l}\right.$ are well-defined analytic vector fields on $U_{\imath l}$. On the other hand the definition of $S\left(X_{i}, l\right)$ shows that $\left|v_{k i l}\right|$ do not vanish and that $v_{1 i l x}$ and $v_{z l l \varsigma}, x \leqq U_{i l}$, do not point the opposite directions each other. Hence $\xi_{i l}$ also is a well-defined non-singular analytic vector field. By (5.3.5), $U_{\imath \imath}$ is the intersection of $I_{l}$ and a neighborhood of $Y_{i l}$ in $X_{i} \cup Y_{\imath \imath}$. Clearly $(5.4 .1,2)$ follow from the above definition of $\xi_{\imath l}$. Hence (5.4) is proved.

(5.5) Under the same notations as (5.4), let $U_{\imath l}^{\prime}$ and $U_{\imath l}^{\prime \prime}$ be open subsets of $X_{i}$ having the same property as $U_{i l}$, namely being the intersections of $X_{i}$ and neighborhoods of $Y_{i l}$ in $X_{\imath} \cup Y_{i l}$, and moreover satisfying:

$$
\bar{U}_{\imath \iota}^{\prime} \cap X_{i} \subset U_{i l}^{\prime \prime}, \bar{U}_{\imath l}^{\prime \prime} \cap X_{\iota} \subset U_{\imath l} \text {. }
$$

Put $U_{i}=\underset{l>\operatorname{codim} I_{2}}{L_{l}} U_{\imath l}^{\prime}$. Then there exists a $C^{\infty}$ vector field $\tilde{\xi}_{\imath}$ on $Y_{\imath}$ such that

(5.5.1) for any $x \subseteq U_{\imath l}^{\prime}, \xi_{i x}$ is contained in $\boldsymbol{R}^{\imath}$, and

(5.5.2) $\xi_{i} f_{k}, k=1,2$, are positive on $U_{\iota}$.

Proof. Let $g_{l}$ be $C^{\infty}$ functions on $X_{i}$ such that $g_{l}=1$ on $L_{\imath l}^{\prime}$, $=0$ on $X_{l}-$ $U_{\imath l}^{\prime \prime}$ and $0<g<1$ on $U_{i l}^{\prime \prime}-\bar{U}_{i l}^{\prime}$. Then, putting $g_{l} \xi_{\imath l x}=0$ on $X_{\imath}-U_{\imath l}$, we extend $g_{l} \xi_{i l}$ to $X_{i}$ as $C^{-3}$ vector fields. Put

$$
\xi_{i x}=\sum_{l>\operatorname{codim}} \prod_{i} \prod_{l^{\prime}<l}\left(1-g_{l^{\prime}}\right) g_{l} \xi_{i l x} .
$$

Then $\xi_{i}$ is a $C$-vector field on $X_{i}$. We will see that $\xi_{\imath}$ satisfies $(5.5 .1,2)$.

For (5.5.1), let $x$ be a point of $U_{i l_{1}}^{\prime}$. Since $1-g_{l_{1}}(x)=0$, we have

$$
\prod_{l^{\prime}<l_{2}}\left(1-g_{l^{\prime}}\right) g_{l_{2}} \xi_{i l_{2} r}=0
$$

for any $l_{2}>l_{1}$. Hence

$$
\xi_{i x}=\sum_{l \leqq l_{1}} \prod_{l^{\prime}<l}\left(1-g_{l^{\prime}}\right) g_{l} \xi_{l l . c} .
$$

By (5.4.1), $g_{l} \xi_{l l . .}$ is contained in $\mathbb{R}^{l_{1}}$ for any $l \leqq l_{1}$. Hence (5.5.1) follows.

For (5.5.2), consider a point $x \in U_{1} \cap \bar{U}_{i l_{1}}^{\prime}$. We can assume $x \notin \bar{U}_{\imath l}^{\prime}$ for any $l<l_{1}$. Since $g_{l} \xi_{l}, f_{k}(x)$ and $1-g_{l}(x)$ are non-negative for any $l, k$, it is sufficient to see

$$
\prod_{l<l_{1}}\left(1-g_{l}\right) g_{l_{1}} \xi_{l l_{1}} f_{k}(x),
$$

$k=1,2$, a se positive. By the above assumption on $x$ and the property of $g_{\iota}$, we have

$$
\left(1-g_{l}(x)\right)>0 \text { for } l<l_{1} \text {, }
$$


and

$$
g_{l_{1}}(x)>0 .
$$

These inequalities together with (5.4.2) imply that (5.5.3) are positive. Hence (5.5) is proved.

(5.6) For any $X_{\imath}$ of codimension $l$ contained in $Z$, let $T^{-}$, be an open neighborhood of $X_{i}$ in $Y$ such that $V_{\imath} \cap X_{\jmath} \subset U_{\jmath l}^{\prime}$ for any $X_{j}$ with $I_{\jmath}=Z$ and $\bar{X}_{j} \supset X_{\imath}$. We denote by $V$ the union of all $V_{i}$. Then $V$ is an open neighborhood of $Z$ in $X$. Considering $X \cap V,\left\{X_{\imath} \cap V\right\}$ in place of $X,\left\{X_{\imath}\right\}$ respectively. we reduce Proposition 5.1 to the next proposition.

Proposition 5.1'. Let $X$ be a locally closed subset of $\mathbb{R}^{n}$. \{.., be a Whitney stratification of $Y, f_{1}$ and $f_{2}$ be the restrictions to $X$ of $C^{\circ}$ inuctions $\tilde{f}_{1}$ and $\tilde{f}_{2}$ on $\boldsymbol{R}^{n}$ respectively, $\left\{\xi_{\imath}\right\}$ be a vector field on $\left\{X_{\imath}\right\}$, and $V_{i}$ be an upen neighborhood of each $X_{i}$ in $X$ such that

(5.6.1) $f_{1}^{-1}(0)=f_{2}^{-1}(0)$,

(5.6.2) $Z=f_{1}^{-1}(0)$ is closed in $\boldsymbol{R}^{n}$,

(5.6.3) $\left\{X_{i}\right\}$ is compatible with $Z$,

(5.6.4) for each $i, \bar{X}_{i} \cap Z \neq \varnothing$,

(5.6.5) for each $i, k$ and $x \in \mathbb{R}^{n}, X_{i}$ and $x+\boldsymbol{R}^{k}$ are transversai if $i \geqq \operatorname{codim} X_{l}$,

(5.6.6) for each $i, j$ and $x \in X_{\imath} \cap V_{3}$ with $X_{i} \not \subset Z, X, \subset Z$ and $\overline{\mathrm{I}}_{\imath} \beth \mathrm{I}_{.} . \xi_{i x}$ is contained in $R^{l}$ where $l=\operatorname{codim} X_{j}$, and

(5.6.7) $\xi_{l}\left(\left.f_{k}\right|_{\mathrm{r}_{l}}\right)$, for any $i, k$ with $X_{\imath} \not \subset Z$, is positive.

Then there exist neighborhoods $W_{1}, W_{2}$ of $Z$ in $X$ and a liumeomorphism =: $W_{1} \rightarrow W_{2}$ such that $f_{2} \circ \tau=f_{1}$ on $W_{1},\left.\tau\right|_{Z}=i d e n t$, and $\tau\left(W_{1} \cap X_{2}\right)=T_{2}-Y_{2}$ for each $i$.

(5.7) Proof of Proposition 5.1'. For the sake of simplicity tre assume $f_{1}$, $f_{2} \geqq 0$. The general case requires no more than complicated notations. For each $X_{i} \subset Z$, there obviously exists an open neighborhood $V_{\imath}^{\prime}$ of $X_{\iota}$ in $X$ such that $\bar{V}_{i}^{\prime}-V_{\imath} \subset \bar{X}_{\imath}-X_{i} \subset Z$. Let $J$ be the index subset consisting of $i$ with $X_{i} \not \subset Z$. Apply Lemma 4.14 to $\left\{X_{i}\right\}_{i \in J}, X^{*}=X-Z, f_{1}, f_{2}, \xi=\left\{\xi_{\imath}\right\}_{\imath \subseteq I} \cdot\left\{\bigcup_{\substack{c i m \\ i \in J^{\prime}}} V_{\imath}-X_{\imath}\right\}_{\text {, }}$

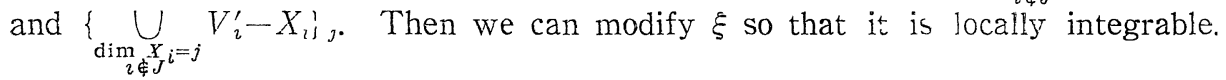
Here we have to replace $X$ by $X \cap \bigcup_{i \notin J} V_{\imath}^{\prime}$ and the belonging $\left.x \equiv I_{\imath}\right\urcorner I^{r}$ in (5.6.6) by $x \in Y_{\iota} \cap V_{j}^{\prime}$. But the replacement does not influence the conclusion of Proposition $5.1^{\prime}$. So we add the assumption that $\xi$ is locally integrable to the assumptions of Proposition 5.1'. Put

$$
c_{\iota}=\inf _{x \in \bar{X}_{i-I}}\left\{\tilde{f}_{1}(x), \tilde{f}_{2}(x)\right\}, i \in J
$$


If $\bar{X}_{i}-X=\varnothing$ (we see later this is not the case), then we put $c_{\imath}=\infty$. Then $c_{i}$ are positive by (5.6.2), the local closedness of $X$ and by the boundedness of $X_{i}$. Let $\Theta: D \rightarrow X^{*}=X-Z, D \subset X^{*} \times \boldsymbol{R}$, be the flow of $\xi$ (4.12). Then $D$ is open, $\Theta$ is continuous, and for any $x \in X^{*}$ we have $t_{x}^{-}<0<t_{. x}^{+}$(may be infinity) such that

$$
D \cap(x \times \boldsymbol{R})=x \times\left(t_{x}^{-}, t_{. x}^{+}\right) .
$$

It follows from $(\overline{0} .6 .7)$ that $\left.\left.f_{0} \circ \Theta\right|_{x_{0},\left(t_{x_{0}},{ }^{+}\right.} ^{+}\right)$is strictly increasing for each $x_{0}$ $\in X_{i}, i \in J$, and each $j$.

Moreover if $f_{j}\left(x_{0}\right)<c_{i}$, then $f_{\mathrm{j}} \circ \Theta\left(x_{0}, t\right)$ converges to 0 , a number larger or equal to $c_{i}$ as $t \rightarrow t_{x_{0}}^{-}+0, t_{x_{0}}^{+}-0$ respectively. In fact, if not so, we have by (5.6.7) and $b y$ the boundedness of $X_{i}$ a sequence $\left\{t_{k}\right\}$ of numbers in $\left(t_{x_{0}}^{-}, t_{x_{0}}^{+}\right)$ such that $\left\{\Theta\left(x_{0}, t_{k}\right)\right\}$ converges to a point $y$ of $\left(\bar{X}_{\imath}-X_{i}\right)-Z-\left(\bar{X}_{\imath}-X\right) \subset X^{*}-X_{i}$. Since $D$ is open, we have a neighborhood $O_{y}$ of $y$ in $X$ and $\varepsilon>0$ such that $D \supset O_{y} \times[-\varepsilon, \varepsilon]$. This means that for sufficiently large $k, \Theta\left(x_{0}, t_{k}\right) \in O_{y}$ and hence $\left(x_{0},\left[t_{i}-s, t_{i}+\varepsilon\right]\right) \subset D$. Hence $t_{k} \rightarrow \infty$ or $-\infty$ as $k \rightarrow \infty$. By the continuity of $\Theta,\left\{\Theta\left(x_{0}, t_{k} \pm \varepsilon\right)\right\}_{k}$ converges to $\Theta(y, \pm \varepsilon)$. Hence $\left\{f_{\jmath} \circ \Theta\left(x_{0}, t_{k} \pm \varepsilon\right)\right\}_{k}$ converges to $\dot{\tau}^{j} \circ \Theta(y, \pm \varepsilon) \neq f_{j}(y)$. This contradicts the fact that $f_{j} \circ \Theta_{x_{0}<\left(t_{x_{0}}^{-}, t_{x_{0}}^{+}\right)}$ is increasing. Since $f_{j}$ is bounded on $X_{\imath}$, we have seen also $\bar{X}_{\imath}-X=\varnothing$ and hence $c_{i} \neq c 0$.

The above property of $f_{\mathrm{o}} \odot \Theta\left(x_{0}, t\right)$ shows that for each $x_{0} \in X_{l}, i \in J$, and each $j$ with $f_{3}\left(x_{0}\right)<c_{\imath}, f_{j} \circ \Theta$ maps diffeomorphically $\left(x_{0} \times\left(t_{x_{0}}, t_{x_{0}}^{\perp}\right)\right) \cap\left(f_{j} \circ \Theta\right)^{-1}\left(\left(0, c_{i}\right)\right)$ onto $\left(0, c_{\imath}\right)$. Hence we uniquely obtain a $C^{\infty}$ diffeomorphism $\tau_{x_{0}}$ from $\Theta\left(\left(x_{0} \times\right.\right.$ $\left.\left.\left(t_{x_{0}}, t_{x_{0}}^{+}\right)\right) \cap\left(f_{1} \circ \Theta\right)^{-1}\left(\left(0, c_{i}\right)\right)\right)$ to $\Theta\left(\left(x_{0} \times\left(t_{x_{0}}^{-}, t_{x_{0}}^{+}\right)\right) \cap\left(f_{2} \circ \Theta\right)^{-1}\left(\left(0, c_{\imath}\right)\right)\right)$ such that $f_{2} \circ \tau_{x_{0}}$ $=f_{1}$ on the domain of definition of $\tau_{x_{0}}$. We remark that $\tau_{x_{0}} \equiv \tau_{x_{0}^{\prime}}$ if both $x_{0}$ and $x_{0}^{\prime}$ are contained in one integral curve of $\tilde{\xi}_{\imath}$. Put

$$
\begin{aligned}
& \Pi_{j l}=\left\{x \in X_{\imath} \mid f_{\imath}(x)<c_{\imath}\right\} \quad \text { for } j=1,2 \text { and } i \subseteq J, \\
& \Psi_{\jmath}=\bigcup_{\imath} W_{\jmath \imath} \cup Z .
\end{aligned}
$$

Then $W_{1}$ is the union of $Z$ and the domains of definition of $\tau_{c_{0}}$ for all $x_{n} \in X_{2}$, $i \in J$, with $\hat{f}_{1}\left(x_{1}\right)<c_{l}, W_{2}$ is the union of $Z$ and all the images of $\tau_{x_{0}}$ 's, and they are both neighborhoods of $Z$ in $X$. We define a map $\tau: W_{1} \rightarrow W_{2}$ by

$$
\tau(x)= \begin{cases}\tau_{x}(x) & \text { for } \quad x \in W_{1}-Z \\ x & \text { for } \quad x \in Z .\end{cases}
$$

Then clearly $=$ is well-defined and one-to-one, we have $f_{2}^{\circ} \tau=f_{1}$ on $W_{1}$ and $\tau=$ ident on $Z$, and for any $\left.i \tau\right|_{W_{1} \cap X_{2}}$ is a $C^{\infty}$ diffeomorphism onto $W_{2} \cap X_{i}$.

We only need to see that $\tau$ is a homeomorphism. Let $\left\{x_{k}\right\}$ be a sequence of points in $H_{1^{\prime}} \cap X_{l}$ converging to $y_{1} \in W_{1} \cap X_{i^{\prime}}$. We want to see that $\left\{\tau\left(x_{k}\right)\right\}$ converges to $\tau\left(y_{1}\right)$. If $i=i^{\prime}$ or $i \notin J$, it is trivial by the continuity of $\left.\tau\right|_{W_{1} \cap x_{i}}$ and by the definition of $\left.\tau\right|_{z}$. Hence we assume $i \neq i^{\prime}$ and $i \in J$. The case $i^{\prime} \notin J$ clearly follows from the next statement. 
(5.7.1) For each $j \notin J$ and for any $\varepsilon>0$ there exists a neighborhood $Q_{\jmath}$ of $X_{j}$ in $X$ such that

$$
\operatorname{dist}(x, \Theta(x, t)) \leqq \varepsilon \quad \text { for } \quad x \in Q_{3}-Z, t \in\left(t_{x}^{-}, 0\right) .
$$

We prove (5.7.1) by induction on codimension of $X_{\jmath}$. (5.7.1) is trivial if $\operatorname{codim} X_{\jmath}=0$. Hence, assume (5.7.1) for $\operatorname{codim} X_{\jmath}<l$. Let $\varepsilon>0$, and for each $j^{\prime} \notin J$ with $\operatorname{codim} X_{\jmath^{\prime}}<l$, let $Q_{\jmath^{\prime}}$ be a neighborhood of $X_{\jmath^{\prime}}$ in $X$ such that

$$
\operatorname{dist}(x, \Theta(x, t)) \leqq \varepsilon / 3 \quad \text { for } \quad x \in Q_{\jmath^{\prime}}-Z, t \in\left(t_{\alpha}^{-}, 0\right) \text {. }
$$

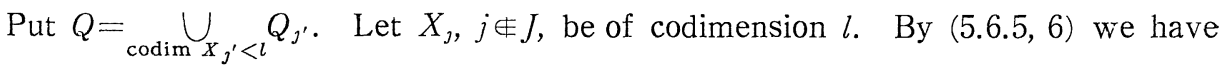
an open neighborhood $Q_{\jmath}^{\prime}$ of $X_{j}$ in $\boldsymbol{R}^{n}$ and a $C^{\infty}$ fiber bundle $\pi_{\jmath}: Q_{\jmath}^{\prime} \rightarrow X_{\jmath}$ such that

where

$$
\begin{aligned}
\left.\pi_{\jmath}\right|_{x_{j}}= & \text { ident, } \\
& \pi_{\jmath}^{-1}(y) \subset\left(y+\boldsymbol{R}^{l}\right) \cap B_{\varepsilon / 3}(y) \quad \text { for } y \in X_{\jmath}, \text { and } \\
& \xi_{i x} \in \boldsymbol{R}^{l} \quad \text { for } \quad x \in X_{\imath} \cap Q_{\jmath}^{\prime}, i \in J,
\end{aligned}
$$

$$
B_{\varepsilon / 3}(y)=\left\{y^{\prime} \in \boldsymbol{R}^{n}|| y-y^{\prime} \mid \leqq \varepsilon / 3\right\} .
$$

We remark that

(5.7.4) if $x_{0} \in X^{*}$ and $t_{1}<t_{2} \in \boldsymbol{R}$ satisfy $\Theta\left(x_{0},\left(t_{1}, t_{2}\right)\right) \subset Q_{\jmath}^{\prime}$, then $\pi_{\jmath} \circ \Theta\left(x_{0},\left(t_{1}, t_{2}\right)\right)$ $=\mathrm{a}$ point.

If necessary, shrink $Q$ so small that for any $y \equiv X_{j}, \lambda(y)=\overline{\pi_{j}^{-1}(y)}-Q_{j}^{\prime}-Q$ is not empty. Put

$$
\begin{aligned}
& d(y)=\inf _{x \in \lambda(y)} f_{1}(x), \\
& Q_{\jmath}=\left\{x \in X \cap Q_{\jmath}^{\prime} \mid f_{1}(x)<d \circ \pi_{\jmath}(x)\right\} .
\end{aligned}
$$

Then we see easily $d(y)>0$ and that $Q_{j}$ is a neighborhood of $X$, in $X$.

Moreover $Q$, satisfies (5.7.1)'. In fact, let $x \in Q,-Z$. Then there are two cases,

$$
\begin{aligned}
& \Theta\left(x,\left(t_{x}^{-}, 0\right)\right) \subset Q_{\jmath}^{\prime} \text { and } \\
& \Theta\left(x,\left(t_{x}^{-}, 0\right)\right) \not \subset Q_{\jmath}^{\prime} .
\end{aligned}
$$

If (i), we have by $(5.7 .3,4)$

$$
\operatorname{dist}(x, \Theta(x, t)) \leqq 2 \varepsilon / 3 \quad \text { for any } t \in\left(t_{x}^{-}, 0\right),
$$

which proves (5.7.1). Now consider the case of (ii). Let $t_{0}$ be the infericr of $t$ with $\Theta(x,(t, 0)) \subset Q_{j}^{\prime}$. Then we see by $(5.7 .3,4)$

$$
\begin{aligned}
& \Theta\left(x,\left(t_{0}, 0\right)\right) \subset \pi_{\jmath}^{-1}\left(\pi_{\jmath}(x)\right) \text { and } \\
& \Theta\left(x, t_{0}\right) \in \overline{\pi_{\jmath}^{-1}\left(\pi_{\jmath}(x)\right)}-Q_{\jmath}^{\prime} \text { hence }
\end{aligned}
$$




$$
\operatorname{dist}(x, \Theta(x, t)) \leqq 2 \varepsilon / 3 \quad \text { for } \quad t \in\left[t_{0}, 0\right) \text {. }
$$

Moreover

Indeed, if not so, then

$$
\Theta\left(x, t_{0}\right) \in Q
$$

$$
\Theta\left(x, t_{0}\right) \in \overline{\pi_{j}^{-1}\left(\pi_{j}(x)\right)}-Q_{j}^{\prime}-Q .
$$

Hence, by the definition of $d(y)$

$$
d \circ \pi_{\jmath}(x) \leqq f_{1} \circ \Theta\left(x, t_{0}\right) .
$$

But, since $f_{1}(x)<d \circ \pi_{j}(x)$ and since $f_{1}$ is strictly increasing on $\Theta\left(x,\left(t_{x}^{-}, 0\right)\right)$, we have

$$
f_{1} \circ \Theta\left(x, t_{0}\right)<d \circ \pi_{\jmath}(x),
$$

which is a contradiction. Hence $\Theta\left(x, t_{0}\right) \in Q$. This implies by (5.7.2)

$$
\operatorname{dist}\left(\Theta\left(x, t_{0}\right), \Theta(x, t)\right) \leqq \varepsilon / 3 \quad \text { for } t \in\left(t_{x}^{-}, t_{0}\right),
$$

which together with (5.7.5) proves that $Q_{j}$ satisfies (5.7.1)'.

Now the case $i^{\prime} \in J$ remains in the proof of the convergence of $\left\{\tau\left(x_{k}\right)\right\}$ to $\tau\left(y_{1}\right)$. Let $t_{1} \in\left(t_{y_{1}}, t_{y_{1}}^{+}\right)$satisfy $\Theta\left(y_{1}, t_{1}\right)=\tau\left(y_{1}\right)$, and let $\varepsilon$ be a small positive number. Then, by the local integrability of $\xi$ there exist neighborhoods $R^{\prime} \subset R$ of $y_{1}$ in $X$ and $\delta>0$ such that

$$
\begin{aligned}
& R \times\left[t_{1}-\delta, t_{1}+\delta\right] \subset D, \\
& f_{2} \circ \Theta\left(x, t_{1}-\delta\right)<f_{2} \circ \Theta\left(y_{1}, t_{1}\right)=f_{2} \circ \tau\left(y_{1}\right) \\
& =f_{1}\left(y_{1}\right)<f_{2} \circ \Theta\left(x, t_{1}+\delta\right) \text { for any } x \in R^{\prime}, \\
& \left|\Theta(x, t)-\tau\left(y_{1}\right)\right|<\varepsilon \text { for }(x, t) \in R \times\left[t_{1}-\delta, t_{1}+\delta\right] .
\end{aligned}
$$

By the definition of $\tau$ there exists a number $\delta_{k}$ for each $k$ such that

$$
\Theta\left(x_{k}, t_{1}+\delta_{k}\right)=\tau\left(x_{k}\right) .
$$

Since $\left\{x_{k}\right\}$ converges to $y_{1}$, and since $f_{2} \circ \tau\left(x_{k}\right)=f_{1}\left(x_{k}\right),(5.7 .6,8)$ show that for sufficiently large $k$

$$
f_{2} \circ \Theta\left(x_{k}, t_{1}-\delta\right)<f_{2} \circ \Theta\left(x_{k}, t_{1}+\delta_{k}\right)<f_{2} \circ \Theta\left(x_{k}, t_{1}+\delta\right),
$$

which implies that $\left|\delta_{k}\right|<\delta$. Therefore it follows from $(5.7 .7,8)$ that

$$
\left|\tau\left(x_{k}\right)-\tau\left(y_{1}\right)\right|<\varepsilon \text {. }
$$

Namely $\left\{\tau\left(x_{k}\right)\right\}$ converges to $\tau^{\prime}\left(y_{1}\right)$. As the continuity of $\tau^{-1}$ is shown in the same way, we complete the proof of Proposition 5.1'.

Remark 5.8. In Proposition 5.1, given a locally finite family $\left\{Y_{j}\right\}$ of subanalytic subsets of $\boldsymbol{R}^{n}$, we can choose $\tau: W_{1} \rightarrow W_{2}$ so that $\tau\left(W_{1} \cap Y_{j}\right)=W_{2} \cap Y_{j}$ for any $j$. In fact, using (2.11), we can construct the Whitney stratification 
$\left\{X_{\imath}\right\}$ of $X$ in (5.3) so that it is compatible with $\left\{Y_{j}\right\}$. Then $\tau$ defined in (5.7) automatically satisfies $\tau\left(W_{1} \cap Y_{j}\right)=W_{2} \cap Y_{j}$ for any $j$.

Remark 5.9. We can refine Proposition 5.1 as follows. For any small neighborhood $W_{3}$ of $Z$ in $X$ there exists moreover a homeomorphism $\tilde{\tau}$ of $X$ such that $\left.\tilde{\tau}\right|_{W_{1}}=\tau$ and $\left.\tilde{\tau}\right|_{x-W_{3}}=$ ident, here $W_{1}$ and $W_{2}$ are chosen to be contained in $W_{3}$.

Proof. (Continued from (5.7).) Let $\Lambda$ be the quotient topological space of $W_{1}-Z$ under the equivalence relation $x \sim \Theta(x, t)$ for any $t$, and let $\theta_{1}: W_{1}-Z$ $\rightarrow \Lambda$ be the canonical surjection, namely $\Lambda$ is an orbit space. Then for any finite subset $J^{0}$ of $J$, we have a positive number $c^{0}$ by the local integrability of $\xi$ such that $c^{0}<c_{i}$ for any $i \in J^{0}$ and that the restriction of $\theta_{1}$ to $\bigcup_{i \in J^{0}} X_{i} \cap f_{1}^{-1}\left(c^{0}\right)$ is homeomorphic to the image $\theta_{1}\left(W_{1} \cap \bigcup_{i \in J^{0}} X_{\imath}\right)$. Repeat this argument for a sequence $\left\{J^{j}\right\}_{j}$ of finite subsets of $J$ such that $\left\{\bigcup_{i \in J} X_{2}\right\}_{J}$ is a locally finite open covering of $X^{*}$. Then let $\left\{c^{\jmath}\right\}$ be the consequent numbers, and let $c(y)$ be a positive continuous function on $A$ such that $c \circ \theta_{1}(x)<c^{j}$ for $x \in X_{i}, i \in J^{j}$. Here the existence of such $c$ follows easily from the remarks that $\left\{\theta_{1}\left(W_{1} \cap \bigcup_{\imath \in J} X_{i}\right)\right\}_{j}$ is a locally finite open covering of $\Lambda$ and that $\Lambda$ is a normal space. Then it follows that the restriction of $\theta_{1}$ to $\left\{x \in W_{1}-Z \mid f_{1}(x)=c \circ \theta_{1}(x)\right\}$ is a homeomorphism onto 1 .

Choose $c(y)$ so small that $W^{r}$, contains

$$
W_{3}^{\prime}=Z \cup\left\{x \leqq W_{1}-Z \mid f_{1}(x) \leqq c \circ \theta_{1}(x)\right\},
$$

and let $c^{\prime}(y)$ be a positive continuous function on $\Lambda$ such that $c^{\prime}\left(y^{\prime}\right)<c(y)$ and that $\tau\left(W_{1}^{\prime}\right)$ is contained in the interior of $W_{3}^{\prime}$ in $X$ where

$$
W_{1}^{\prime}=Z \cup\left\{x \equiv W_{1}-Z \mid f_{1}(x) \leqq c^{\prime} \circ \theta_{1}(x)\right\} .
$$

Put $\quad W_{2}^{\prime}=\tau\left(W_{1}^{\prime}\right), \theta=\left(\theta_{1}, \int_{1}\right): W_{3}^{\prime}-Z \longrightarrow, 1 \times(0, \infty)$.

Then $\theta$ is a homeomorphism onto the image, and we have

$$
\begin{aligned}
& \theta\left(W_{3}^{\prime}-Z\right)=\{(y, t) \in \Lambda \times(0, \infty) \mid t \leqq c(y)\}, \\
& \theta\left(W_{1}^{\prime}-Z\right)=\left\{t \leqq c^{\prime}(y)\right\}, \\
& \theta\left(W_{2}^{\prime}-Z\right) \subset \operatorname{Int} \theta\left(W_{3}^{\prime}-Z\right) .
\end{aligned}
$$

Since each integral curve of $\xi$ is invariant under $\tau$, the homeomorphism $\tau^{\prime}$ : $\theta\left(W_{1}^{\prime}-Z\right) \rightarrow \theta\left(W_{2}^{\prime}-Z\right)$ defined by $\tau^{\prime} \circ \theta=\theta \circ \tau$ is in the form $\tau^{\prime}(y, t)=\left(y, \tau^{\prime \prime}(y, t)\right)$ for some continuous function $\tau^{\prime \prime}$. Let $y \in \Lambda$ be fixed. Then $\tau^{\prime \prime}(y, t)$ is a homeomorphism from $y \times\left\{t \in(0, \infty) \mid t \leqq c^{\prime}(y)\right\}$ to $\theta\left(W_{2}^{\prime}-Z\right) \cap y \times \boldsymbol{R}$. Hence we can extend $\tau^{\prime \prime}(y, t)$ to a homeomorphism of $y \times\{t \leqq c(y)\}$ so that it is linear on the 
complement of $y \times\left\{t \leqq c^{\prime}(y)\right\}$. Let the extension be denoted by $\tilde{\tau}_{y}^{\prime \prime}$. Then clearly

$$
\tilde{\tau}^{\prime}(y, t)=\left(y, \tilde{\tau}_{y}^{\prime \prime}(t)\right) \quad \text { for } \quad(y, t) \in \theta\left(W_{3}^{\prime}-Z\right)
$$

is a homeomorphism of $\theta\left(W_{3}^{\prime}-Z\right)$ such that

$$
\tilde{\tau}^{\prime}=\left\{\begin{array}{l}
\tau^{\prime} \text { on } \theta\left(W_{1}^{\prime}-Z\right) \\
\text { ident on }\{(y, t) \in \Lambda \times(0, \infty) \mid t=c(y)\} .
\end{array}\right.
$$

Hence the extension $\tilde{\tau}$ of $\tau$ induced by $\tilde{\tau}^{\prime}$ is a homeomorphism of $W_{3}^{\prime}$ which is the identity on the boundary of $W_{3}^{\prime}$ in $X$. Therefore we can extend $\approx$ moreover to $X$ so that it is the identity outside $W_{3}^{\prime}$. After replacing $W_{1}, W_{2}$ by $W_{1}^{\prime}, W_{2}^{\prime}$ respectively, we obtain the remark.

Remark 5.10. In Proposition 5.1, $\tau$ is the identity on $\left\{x \in W_{1} \mid f_{1}(x)=f_{2}(x)\right\}$. In Remark 5.9, assume $f_{1}=f_{2}$ on a neighborhood of a closed subset $C$ of $X$. Then $\tilde{\tau}$ can be chosen to be the identity on a neighborhood of $C$. These are clear by the method of construction $\tau$ and $\tilde{\tau}$.

Remark 5.11. In Proposition 5.1 and Remarks 5.9, 10, assume moreover that $X$ is an analytic manifold and that $f_{1}, f_{2}$ are analytic. Then $\tau$ and $\tilde{\tau}$ can be chosen to be analytic and $C^{\infty}$ differentiable on $X-Z$ respectively.

Proof. Come back to (5.3). The stratification $\left\{X_{i}\right\}$ can be chosen so that $\left\{Y_{2}-Z\right\}$ is the family of all connected components of $X-Z$. Then $\Theta: D \rightarrow X^{*}$ in (5.7) turns out to be of class $C^{\infty}$. Here the strata may fail to satisfy the boundedness condition. But the condition is used only in the construction of $W_{i}$ in (5.7), namely of $c_{i}$. In case in which $X$ is an analytic manifold and $f_{1}$ and $f_{2}$ are analytic, it is clear that there exists a continuous function $c(y)$ on $\Lambda$ in the proof of Remark 5.9, since $\Theta$ is differentiable. Replace $W_{i}$ by $W_{\imath}^{\prime}$ of the proof of Remark 5.9. Then the $C^{\infty}$ differentiability of $\tau$ is trivial by the definition of $\tau$. About $\tilde{\tau}$, it is not of class $C^{\infty}$ at

$$
\theta^{-1}\left\{(y, t) \in \Lambda \times(0, \infty) \mid t=c(y) \text { or }=c^{\prime}(y)\right\} .
$$

To make $\tilde{\tau}$ differentiable, at first choose $c(y), c^{\prime}(y)$ to be differentiable. It has a meaning since $\Lambda$ naturally has a $C^{\infty}$ manifold structure induced by $X$. Nextly we modify $\tilde{\tau}$ to be differentiable. It is easy because of the differentiability of $\Theta$ and since we only need to consider $\tilde{\tau}^{\prime}$ on $\{(y, t) \in \Lambda \times(0, \infty) \mid t \leqq c(y)\}$. We omit the details.

For an analytic modification of $\tau$, we apply Theorem 8.4 in [16] to $f_{2} \circ \tau_{1}, f_{1}$ on $W_{1}-Z$ where $\tau_{1}: W_{1}-Z \rightarrow W_{2}-Z$ is an analytic approximation diffeomorphism of $\left.\tau\right|_{W_{1}-Z}$ in the Whitney topology [5]. Then there exists an analytic diffeomorphism $\tau_{2}: W_{1}-Z \rightarrow W_{1}-Z$ such that $f_{1}=f_{2} \circ \tau_{1} \circ \tau_{2}$. Moreover $\tau_{1} \circ \tau_{2}$ can be chosen to be arbitrarily close to $\left.\tau\right|_{W_{1}-Z}$ in the Whitney topology by Remark 8.5 in [16]. Hence, if we extend $\tau_{1}{ }^{\circ} \tau_{\text {。 }}$ to $W_{1}$ by putting $\tau_{1} \circ \tau_{2}=$ ident on $Z$, 
then the extension is a homeomorphism from $W_{1}$ to $W_{2}$ which satisfies the requirement of Remark 5.11 .

Problem. In Proposition 5.1, can $\tau$ be subanalytic?

Proof of Proposition 5.2. Let $M \subset \boldsymbol{R}^{n}$, and let $b$ be a number contained in $f_{1}(S)$. Put $S^{\prime}=f_{1}^{-1} f_{1}(S)$. By the definition, $S$ is identical with $S(M, n)$ defined in (5.3). Clearly $S(M, n)$ is closed, and (5.3.7) implies that $S(M, n)-f_{1}^{-1}(b)$ is empty in a neighborhood of $f_{1}^{-1}(b)$. Hence it follows that $f_{1}(S)$ is a discrete set and hence that $S^{\prime}$ is an analytic set.

Let $A$ be a connected component of $M-S^{\prime}$. Then we have $a_{1}, a_{2} \in \bar{A}-A$ such that $f_{1}(A)=\left(f_{1}\left(a_{1}\right), f_{1}\left(a_{2}\right)\right)$ since $M$ is compact. We, consequently, have $f_{1}(A)=f_{2}(A)$. Hence, by the proofs of Proposition 5.1 and Remark 5.11, we have neighborhoods $W_{1}, W_{2}$ and $U$ of $S^{\prime}$ in $M$ with $W_{1}, W_{2} \subset U$, a $C^{\infty}$ vector field $\xi$ on $U_{1}=U-S^{\prime}$ and a homeomorphism $\tau: W_{1} \rightarrow W_{2}$ such that $f_{2} \circ \tau=f_{1}$ on $I_{1}, \tau=$ ident on $S^{\prime}$ and $\xi\left(\left.f_{\imath}\right|_{U}\right)>0, i=1,2$ and that $\tau$ is defined separately on each integral curve of $\xi$, namely, $\tau\left(\right.$ curve $\left.\cap W_{1}\right)=$ curve $\cap W_{2}$.

Let $\psi$ be a $C^{\infty}$ function on $M$ such that $\psi=0$ on a neighborhood of $S^{\prime},=1$ on a neighborhood of $M-U$ and $0 \leqq \phi \leqq 1$. Put

$$
\xi^{\prime}=(1-\psi) \xi+\phi\left|d f_{1}\right| d f_{2}+\phi\left|d f_{2}\right| d f_{1} .
$$

Then $\xi^{\prime}$ is a $C^{\infty}$ vector field on $M-S^{\prime}$ satisfying $\xi^{\prime}\left(\left.f_{\imath}\right|_{M-S^{\prime}}\right)>0, i=1$, 2, because of $\left(\left|d f_{1}\right| d f_{2}+\left|d f_{2}\right| d f_{1}\right) f_{i}>0$ on $M-S^{\prime}$ and $\xi^{\prime}=\xi$ on (a neighborhood of $\left.S^{\prime}\right)-S^{\prime}$. In the same way as the construction of $\tau$, we obtain a one-to-one mapping $\tau^{\prime}$ : $M \rightarrow M$ using the integral curves of $\xi^{\prime}$ such that $f_{2}{ }^{\circ} \tau^{\prime}=f_{1}$ on $M$. Then we automatically have $\tau^{\prime}=\tau$ on a neighborhood of $S^{\prime}$, and the differentiability of $\left.\tau^{\prime}\right|_{M-S^{\prime}}$ is clear. Hence $\tau^{\prime}$ is a homeomorphism of $M$. An analytic modification of $\left.\tau^{\prime}\right|_{M-S^{\prime}}$ proceeds just in the same way as the proof of Remark 5.11, and $\tau^{\prime} \mid s^{\prime}$ is the identity. Hence Proposition 5.2 is proved.

We will apply Proposition 5.1 and Remarks 5.9, 10 later in the following form.

Corollary 5.12. Let $Z \subset X \subset \mathbb{R}^{n}$ be polyhedrons, and let $f$ be a subanalytic function on $X$. Assume that $X$ and $Z$ are closed in $\boldsymbol{R}^{n}$ and that for any $x \subseteq Z$, $f^{-1} \int(x) \equiv Z$ as germs at $x$. Then there exists a homeomorphism $\tau$ of $X$ such that $f \circ \tau$ is PL on a neighborhood of $Z$ and that $\tau$ is the identity on $Z$ and outside a given neighborhood of $Z$. Moreover if there is a closed subset $C$ of $X$ such that $f$ is $P L$ on a neighborhood of $C$, then $\tau$ is chosen to be the identity on a neighborhood of $C$.

Proof. Let $L \subset K$ be simplicial complexes such that $|K|=X$ and $|L|=Z$. By subdividing $K$ and by the assumption we can assume that $L$ is full in $K$ (see [13] for the definition), hence that for any $\sigma \in K, f(\sigma \cap L)$ consists of at most one member and that $f^{-1} f(\sigma \cap L) \cap \sigma$ is a simplex of $L$. The last condi- 
tion means that if we define a linear function $f_{1}$ on $\sigma$ by putting $f_{1}=f$ on the vertexes, then $f-f(\sigma \cap L)$ and $f_{1}-f(\sigma \cap L)$ satisfy the conditions of Proposition 5.1 on $\sigma$. Let $f_{1}$ be defined globally on $X$ in this way separately on each simplex. Then $f_{1}$ is a well-defined subanalytic function on $X$. Apply Proposition 5.1 and Remark 5.9 to $f$ and $f_{1}$ on a small neighborhood of each connected component of $Z$. Then we have a homeomorphism $\tau$ of $X$ such that $f \circ \tau=f_{1}$ on a neighborhood of $Z$ and that $\tau$ is the identity outside a given neighborhood of $Z$. Since $f_{1}$ is $P L$, so is $f_{\circ} \tau$ on a neighborhood of $Z$. Thus the first half of the corollary is proved.

For the latter half, subdivide $K$ so that $f$ is linear on any simplex which intersects with $C$. Let $Z^{\prime}$ denote the union of such simplexes. Subdividing $K^{r}$ once more, we can assume moreover that $f$ is linear on each simplex which intersects with $Z^{\prime}$. Then we automatically have $f=f_{1}$ on a neighborhood of $Z^{\prime}$. Hence, by Remark 5.10, $\tau$ can be the identity on a neighborhood of $Z^{\prime}$ and hence of $C$. Therefore Corollary 5.12 is proved.

Remark 5.13. Let $\varepsilon$ be a positive continuous function on $X$ in Remarks 5.9, 10 and Corollary 5.12. Then $\approx$ in Remarks 5.9, 10 and $\tau$ in Corollary 5.12 can be chosen so that

$$
|\tilde{\tau}(x)-x|<\varepsilon(x) \text { and }|\tau(x)-x|<\varepsilon(x) \text { for } x \in X .
$$

It is clear by (5.7.1) and by the method of construction of $\tilde{\tau}$ and $\tau$.

\section{§6. Analytic Triangulations}

At this stage, let us consider the problem of piecewise linearization of an analytic function $f: \boldsymbol{R}^{n} \rightarrow \boldsymbol{R}$. For any point $x \in \boldsymbol{R}^{n}$, the pair $\left(\boldsymbol{R}^{n}, f^{-1} f(x)\right)$ can be triangulated by Proposition 3.1. Hence Corollary 5.12 shows the existence of a homeomorphism $\tau$ of $\mathbb{R}^{n}$ such that $f \circ \tau$ is $P L$ on a neighborhood of $f^{-1} f(x)$. In the extension of this argument to the global $\boldsymbol{R}^{n}$ there are two difficulties. At first the domains where $f$ is piecewise linearized are too narrow to cover $\boldsymbol{R}^{n}$. The other difficulty is that we can not say " $\tau$ is subanalytic". If $\tau$ were subanalytic, then we would repeat the argument above even if the domains of piecewise linearization of $f$ intersect.

We need another method of piecewise linearization. Fortunately we can obtain by Lemma 6.15 below a large domain of piecewise linearization where $f$ is $C^{\infty}$ regular and $z$ is subanalytic. We will show also an analytic triangulation of an analytic manifold (Proposition 6.11). The results in this section are based on $[12]$.

Let $K$ always denote a simplicial complex, $M \subset \boldsymbol{R}^{n}$ an analytic manifold and $r=1, \cdots, \infty$ or $\omega$. For any subcomplex $L$ of $K$, let $N(L, K)$ denote the simplicial neighborhood of $L$ in $K$, namely the subcomplex generated by all $\sigma \in K^{-}$ with $\sigma \cap|L| \neq \varnothing$. 
Definition 6.0. A linear isomorphism $g: K \rightarrow L$ of simplicial complexes is a homeomorphism $g:|K| \rightarrow|L|$ carrying each simplex of $K$ linearly onto one of $L$.

Definition 6.1. A $C^{r}$ map $g: K \rightarrow M$ means a map $g:|K| \rightarrow M$ such that the restrictions $\left.g\right|_{\sigma}, \sigma \in K$, are of class $C^{r}$. We say that $g$ is analytic on a subpolyhedron $P$ of $|K|$ if we have a subcomplex $K_{1}$ of $K^{\prime}$ with $P=\left|K_{1}\right|$ such that $\left.g\right|_{K_{1}}$ is of class $C^{\omega}$. Let $K^{\prime}$ be another simplicial complex with $\left|K^{\prime}\right|=|K|$, and let $g^{\prime}: K^{\prime \prime} \rightarrow M$ be a $C^{r}$ map. We write $g=g^{\prime}$ on $P$ if we have subcomplexes $K_{1}^{\prime}$ of $K$ and $K_{1}^{\prime}$ of $K^{\prime \prime}$ such that $P=\left|K_{1}\right|=\left|K_{1}^{\prime}\right|, K_{1}=K_{1}^{\prime}$ and $\left.g\right|_{K_{1}}=\left.g^{\prime}\right|_{K_{1}^{\prime}}$.

Definition 6.2. For any $b \in|K|$, let $\operatorname{st}\left(b, K^{-}\right)$denote the union of simplexes of $K$ which contain $b$. Let $g: K \rightarrow M \subset \mathbb{R}^{n}$ be a $C^{r}$ map, and let $b \in|K|$. We define $d g_{0}^{*}: \operatorname{st}(b, K) \rightarrow \boldsymbol{R}^{n}$ by

$$
d g_{b}^{*}(x)=d\left(\left.g\right|_{\sigma}\right)_{b}(x-b),
$$

where $\sigma$ is a simplex containing $b+\varepsilon(x-b)$ for small $\varepsilon>0$. Here $K$ is regarded as to be contained in a Euclidean space, and $x-b$ is regarded as a tangent vector at $b$. The definition does not depend on the choice of imbedding of $K$ in a Euclidean space.

Definition 6.3. Let $g: \Lambda \rightarrow M \subset \mathbb{R}^{n}$ be a $C^{r}$ map. We call $g$ an imbedding if $g$ and $d g_{b}^{*}$ are homeomorphic onto the images for any $b \in|K|$. If $g$ is also a homeomorphism onto $M$, it is called a $C^{r}$ triangulation of $M$.

Definition 6.4. Let $g: K \rightarrow \boldsymbol{R}^{n}$ be a $C^{r}$ map. Fix an imbedding of $K$ in a Euclidean space. Let $o$ be a positive continuous function on $|K|$. A map $g^{\prime}$ : $|K| \rightarrow \mathbb{R}^{n}$ is called a $\delta$-approximation of $g$ if

(6.4.1) for some subdivision $K^{\prime}$ of $K, g^{\prime}: K^{\prime \prime} \rightarrow \mathbb{R}^{n}$ is a $C^{r}$ map,

(6.4.2) $\left|g(b)-g^{\prime}(b)\right|<\delta(b)$ for any $b \in|K|$ and if

(6.4.3) $\left|d g_{b}^{*}(x)-d g_{b}^{\prime *}(x)\right| \leqq \delta(b)|x-b|$ for any $b \in|K|, x \in \mathrm{st}\left(b, K^{\prime \prime}\right)$.

We will use the above definitions in the cell complex case too.

Lemma 6.5 (Theorem 8.8, [12], see also the exercise (c) following it). Let s: $K \rightarrow R^{n}$ br a $C^{r}$ imbedding. Then there exists a positive continuous function o on $|K|$ such that any o-approximation of $g$ is an inbedding.

Definition 6.6. Let $g: K \rightarrow \mathbb{R}^{n}$ be a $C^{r}$ map. The secant map induced by 5. $g_{K}: K \rightarrow R^{n}$, is defined by $g_{K}=g$ on the vertex set of $K$ so that it is linear on each simplex of $T$.

Lemma 6.7 (Theorem 9.6, [12]). Lel $g: K \rightarrow R^{r}$ be a $C^{r}$ map, $K$ finite. Given a coisiant function $\delta>0$ on $|K|$, there is a subdinision ${ }^{\prime \prime}$ of $T$ such that the secant mat $g_{K^{\prime}}$ is a o-approxination of $g$. 
Lemma 6.8. Let $g: K \rightarrow \boldsymbol{R}^{n}$ be a $C^{r}$ map. Let $K_{1}$ be a finite subcomplex of $K$. Given $\varepsilon>0$, there is $\delta>0$ such that any $\delta$-approximation $h: K_{1}^{\prime} \rightarrow \boldsymbol{R}^{n}$ of $\left.g\right|_{K_{1}}$ can be extended to an E-approximation $\tilde{h}: K^{\prime} \rightarrow \boldsymbol{R}^{n}$ of $g$ for some subdivision $K^{\prime}$ of $K$ with $\left.K^{\prime}\right|_{\left|K_{1}\right|}=K_{1}^{\prime}$. Here we can choose $\tilde{h}=g$ on $|K|-\left|\stackrel{N}{ }\left(K_{1}, K\right)\right|$ where $\stackrel{N}{N}=N\left(K_{1}, K\right)-\left\{\sigma \in K|\sigma \cap| K_{1} \mid=\varnothing\right\}$.

Proof. If $r \leqq \infty$, the lemma coincides with Lemma 9.8, [12], so assume $r=$ $\omega$. In this case we have to modify the proof in [12].

Special case. Assume $K$ is the complex generated by one simplex $\sigma$, and $K_{1}=K-\{\sigma\}$.

Proof of special case. Let $a$ be the barycenter of $\sigma$. Put

$$
\begin{aligned}
& \sigma_{[b, c]}^{\prime}=\left\{t(x-a)+a \mid x \in \sigma^{\prime}, b \leqq t \leqq c\right\} \quad \text { for } \quad \sigma^{\prime} \in K_{1}^{\prime} \text { and } b, c \in R . \\
& \sigma_{1}^{\prime}=\sigma_{[0,1 / 2]}^{\prime}, \sigma_{2}^{\prime}=\sigma_{[1 / 21 / 2]}^{\prime}, \sigma_{3}^{\prime}=\sigma_{[1 / 2,1]}^{\prime} \quad \text { and } \quad \sigma_{3}=\bigcup_{\sigma^{\prime} \in K_{1}^{\prime}} \sigma_{3}^{\prime} .
\end{aligned}
$$

Let $K^{\prime}$ be the cell complex consisting of $a, \sigma_{1}^{\prime}, \sigma_{2}^{\prime}, \sigma_{3}^{\prime}$ for all $\sigma^{\prime} \in K_{1}^{\prime}$ and $K_{1}^{\prime}$. Let $\rho: K_{1}^{\prime} \cup\left\{\sigma_{2}^{\prime}, \sigma_{3}^{\prime} \mid \sigma^{\prime} \in K_{1}^{\prime}\right\} \rightarrow \partial \sigma$ be the map defined by $\rho(t(x-a)+a)=x, x \in \partial \sigma$. Then $\rho$ is of class $C^{\omega}$. Hence $h \circ \rho$ can be an arbitrarily close approximation of $g \circ o$. Hence if we put

$$
\begin{aligned}
& \alpha(t(x-a)+a)=2(t-1 / 2) \quad \text { for } \quad 1 / 2 \leqq t \leqq 1, x \in \partial \sigma \text { and } \\
& \tilde{h}= \begin{cases}g+(h \circ \rho-g \circ \rho) \cdot \alpha & \text { on } \sigma_{3} \\
g & \text { on } \sigma-\sigma_{3},\end{cases}
\end{aligned}
$$

then $\tilde{h}$ can be an $\varepsilon$-approximation of $g$ such that $\left.\tilde{h}\right|_{\partial \sigma}=h$. Since there is a simplicial subdivision of $K^{\prime}$ fixed on $K_{1}^{\prime}$ (see e.g. [13]), the special case is proved.

The general case proceeds in the same way as [12]. We prove it by induction. Assume a construction of a map $\tilde{h}_{k}:\left|K_{1}\right| \cup\left(|K|-\left|\stackrel{N}{N}\left(K_{1}, K\right)\right|\right) \cup\left|K^{k}\right| \rightarrow \boldsymbol{R}^{n}$ which is at once an approximation of the restriction of $g$ to the domain and an extension of $h$ such that $\tilde{h}_{k}=g$ on $|K|-\left|\stackrel{N}{ }\left(K_{1}, K\right)\right|$, where $K^{k}$ is the $k$-skeleton of $K$, (if $k=0$, it is trivial since $\left|K^{0}\right| \subset\left|K_{1}\right| \cup\left(|K|-\left|\stackrel{N}{N}\left(K_{1}, K\right)\right|\right.$ ).) We want to construct $\tilde{h}_{k+1}$ on $\left|K_{1}\right| \cup\left(|K|-\left(\stackrel{N}{ }\left(K_{1}, K\right) \mid\right) \cup\left|K^{k+1}\right|\right.$. For any $\sigma \in\left(K^{k+1}-K^{k}\right) r_{1}$ $\left(N\left(K_{1}, K\right)-K_{1}\right), \tilde{h}_{k}$ is already defined on $\partial \sigma$. Hence, by the special case, we can extend $\left.\tilde{h}_{k}\right|_{\partial \sigma}$ onto $\sigma$ so that the extension is an approximation of $\left.g\right|_{\sigma}$. Therefore $\tilde{h}_{k}$ has an extension $\tilde{h}_{k+1}$ to $\left|K_{1}\right| \cup\left(|K|-\left|\stackrel{N}{ }\left(K_{1}, K\right)\right|\right) \cup\left|K^{k+1}\right|$ which is an approximation of the restriction of $g$ on the domain. Clearly $\tilde{h}_{k+1}=g$ on $|K|-\left|\stackrel{N}{ }\left(K_{1}, K\right)\right|$. Thus we construct inductively $\tilde{h}=\tilde{h}_{m}$ which satisfies the requirement in Lemma 6.8, where $m$ is the dimension of $N\left(K_{1}, K\right)$. Here the grades of approximation of $\tilde{h}_{k}, k=0, \cdots, m$, to the restriction of $g$ to the domains are decided by downward induction so that $\tilde{h}$ is an $\varepsilon$-approximation of $g$. We omit the details (see [12]). 
Lemma 6.9. Let $g: K \rightarrow M$ be a $C^{r}$ map, $r \neq \omega$. Let $K_{1}$ be a finite subcomplex such that $g\left(\left|N\left(K_{1}, K\right)\right|\right)$ is contained in a coordinate neighborhood of $M$. Let $\varepsilon>0$. Then there exists an $\varepsilon$-approximation $g^{\prime}: K^{\prime} \rightarrow M$ of $g$ such that $g^{\prime}=$ $g$ on $|K|-\left|\stackrel{N}{ }\left(K_{1}, K\right)\right|$ and that $\left.g^{\prime}\right|_{\left|K_{1}\right|}$ is analytic. Moreover if $\left.g\right|_{\left|K_{2}\right|}$ is analytic for some subcomplex $K_{2}$ of $K$, so is $\left.g^{\prime}\right|_{\left|K_{1} \cup K_{2}\right|}$.

Proof. Since we have an analytic coordinate neighborhood of $M$ containing $g\left(\left|N\left(K_{1}, K\right)\right|\right)$, and since $g^{\prime}$ is required to equal $g$ on $|K|-\left|N\left(K_{1}, K\right)\right|$, the problem is reduced to the case $M=\boldsymbol{R}^{n}$. In this case, the first half of the lemma follows from Lemmas $6.7,8$, namely $\left.g^{\prime}\right|_{\left|K_{1}\right|}$ is defined to be the secant map $g_{K_{1}^{\prime}}$ for some subdivision $K_{1}^{\prime}$ of $K_{1}^{r}$, and we let $g^{\prime}$ be its extension to $|K|$ constructed in Lemma 6.8.

For the latter half, we have to check up the above extension of $g_{K_{1}^{\prime}}$. Return to the proof of Lemma 6.8. In the special case, there are two cases $K_{2}=K$ or $K_{2} \subset K_{1}$. If $K_{2}=K, g^{\prime}: K^{\prime} \rightarrow \boldsymbol{R}^{n}$ is analytic because so are $\rho: K_{1}^{\prime} \cup\left\{\sigma_{2}^{\prime}, \sigma_{3}^{\prime} \mid \sigma^{\prime} \leqq\right.$ $\left.K_{1}^{\prime}\right\} \rightarrow \boldsymbol{R}^{n}$ and $\alpha: K_{1}^{\prime} \cup\left\{\sigma_{2}^{\prime}, \sigma_{3}^{\prime} \mid \sigma^{\prime} \in K_{1}^{\prime}\right\} \rightarrow \boldsymbol{R}$. If $K_{2} \subset K_{1}$, the latter half is trivial. Since the general case is treated by stages, and since each stage is equivalent to the special case, the latter half also in the general case is clear.

Lemma 6.10. Let $g: K \rightarrow M$ be a $C^{r}$ map, $r \neq \omega$. Let $\varepsilon$ be a positive continuous function on $|K|$. Then there exists an analytic e-approximation $g^{\prime}: K^{\prime} \rightarrow$ $M$ of $g$. Moreover, let $K_{1} \subset K_{2}$ be subcomplexes of $K$ such that $N\left(K_{1}, K\right) \subset K_{2}$. If $g$ is analytic on $\left|K_{2}\right|$, then u'e can choose $g^{\prime}=g$ on $\left|K_{1}\right|$.

Proof. Let $K_{3}$ be a subcomplex of $K$ such that $\left|K_{3}\right| \cap\left|K_{1}\right|=\varnothing$ and $\left|K_{3}\right| \bigcup$ $\left|K_{2}\right|=|K|$, for example $K_{3}=K-\stackrel{\circ}{N}\left(K_{1}, K\right)$. Subdivide $K$ so that for any $\sigma \in K_{3}, g(|N(\sigma, K)|)$ is contained in some coordinate neighborhood of $M$ and that the restriction of the subdivision to $\left|K_{1}\right|$ remains $K_{1}$. We use the same notation $K$ for the subdivision. Let us order all simplexes of $K_{3}$ as $\sigma_{1}, \sigma_{2}, \cdots$. We will construct $g^{\prime}$ inductively.

Put $g^{-1}=g^{0}=g$. Let $k$ be a non-negative integer. Assume an $\varepsilon / 2^{k}$-approximation $g^{k}: K(k) \rightarrow M$ of $g^{k-1}: K(k-1) \rightarrow M$ such that $g^{k}=g^{k-1}$ on $|K|-\left|\stackrel{N}{ }\left(\sigma_{k}, K\right)\right|$ and that $g^{k}$ is analytic on $\left|K_{2}\right| \cup \bigcup_{\imath=1}^{k} \sigma_{\iota}$, where $K(k)$ is a subdivision of $K(k-1)$ such that $K(-1)=K(0)=K$. Replacing $\varepsilon$ by smaller one, we can assume that the $\varepsilon_{k}$-neighborhood of $g\left(\left|N\left(\sigma_{k}, K\right)\right|\right)$ in $M$ for any $k$ is contained in some coordinate neighborhood of $M$ where $\varepsilon_{k}=\sup _{x \in\left|N\left(\sigma_{k} K^{k}\right)\right|} \varepsilon(x)$. Hence $g^{k}\left(\left|N\left(\sigma_{k+1}, K\right)\right|\right)$ is contained in a coordinate neighborhood of $M$. Apply Lemma 6.9 to $g^{k}$ and the subcomplex of $K(k)$ whose underlying polyhedron is $\sigma_{k+1}$. Then we obtain a subdivision $K(k+1)$ of $K(k)$ and an $\varepsilon / 2^{k+1}$-approximation $g^{k+1}: K(k+1) \rightarrow M$ of $g^{k}$ such that $g^{k+1}=g^{k}$ on $|K|-\left|\stackrel{N}{ }\left(\sigma_{k+1}, K\right)\right|$ and that $g^{k+1}$ is analytic on $\left|K_{2}\right| \cup \bigcup_{i=1}^{k+1} \sigma_{\imath}$. Since

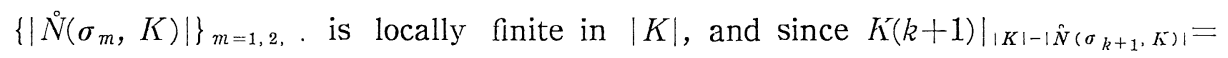


$\left.K(k)\right|_{|K|-\left|{ }^{\circ}\left(\sigma_{k+1}, K\right)\right|}$, the limit of $g^{k}: K(k) \rightarrow M$, as $k \rightarrow \infty$, is an $\varepsilon$-approximation of $g$. Let the limit be $g^{\prime}: K^{\prime} \rightarrow M$. As $|K|$ is covered by $\left|K_{2}\right|$ and $\sigma_{1}, \cdots$, it follows from the definition of $g^{\prime}$ that $g^{\prime}$ is analytic on $|K|$. It is clear that $g^{\prime}=g$ on $\left|K_{1}\right|$ also by the definition. Hence we proved the lemma.

Proposition 6.11. $M$ has a $C^{\omega}$ triangulation.

Proof. By Theorem 10.6, [12] we have a $C^{\infty}$ triangulation $g: K \rightarrow M$. There exists a positive continuous function $\varepsilon$ on $|K|$ such that any $\varepsilon$-approximation of $g$ is an imbedding (Lemma 6.5). Moreover we easily choose $\varepsilon$ so that such an $\varepsilon$-approximation is surjective on $M$, namely, a $C^{\infty}$ triangulation of $M$. Hence Proposition 6.11 follows from Lemma 6.10 .

Remark 6.12. Let $M$ may have boundary. Let $g_{1}: K_{1} \rightarrow M$ and $g_{2}: K_{2} \rightarrow M$ be $C^{1}$ triangulations. Then there are arbitrarily close approximations $g_{1}^{\prime}, g_{2}^{\prime}$ of $g_{1}, g_{2}$ respectively such that $g_{2}^{\prime-1} \circ g_{1}^{\prime}$ is a linear isomorphism (Theorem 10.5, [12]). Hence, if $g: K \rightarrow M$ is a $C^{1}$ triangulation, then $|K|$ is automatically a $P L$ manifold since we have at least one $C^{1}$ triangulation $g_{1}: K_{1} \rightarrow M$ such that $\left|K_{1}\right|$ is a $P L$ manifold (Cairns-Whitehead).

Lemma 6.13. Let $g_{1}: K_{1} \rightarrow M$ and $g_{2}: K_{2} \rightarrow M$ be $C^{r}$ imbeddings whose images are closed in $M$. Let $\varepsilon>0$ be a continuous function on the disjoint union of $\left|K_{1}\right|$ and $\left|K_{2}\right|$. Let $L_{1}, L_{2}$ be subcomplexes of $K_{1}, K_{2}$ respectively such that

$$
\begin{aligned}
& g_{1}\left(\left|N\left(L_{1}, K_{1}\right)\right|\right) \cap g_{2}\left(\left|K_{2}\right|\right)=\varnothing \quad \text { and } \\
& g_{2}\left(\left|N\left(L_{2}, K_{2}\right)\right|\right) \cap g_{1}\left(\left|K_{1}\right|\right)=\varnothing .
\end{aligned}
$$

Then there exist a complex $K$, linear isomorphisms $i_{1}$ and $i_{2}$ from $K_{1}^{\prime}$ and $K_{2}^{\prime}$, subdivisions of $K_{1}$ and $K_{2}$ respectively, to subcomplexes of $K$ and a $C^{r}$ imbedding $g: K \rightarrow M$ such that $g_{1}^{\prime}=g \circ i_{1}, g_{2}^{\prime}=g \circ i_{2}$ are $\varepsilon$-approximations of $g_{1}, g_{2}$ respectively, that $i_{1}\left(\left|K_{1}^{\prime}\right|\right) \cup i_{2}\left(\left|K_{2}^{\prime}\right|\right)=|K|$ and that $g_{1}^{\prime}=g_{1}$ on $\left|L_{1}\right|, g_{2}^{\prime}=g_{2}$ on $\left|L_{2}\right|$.

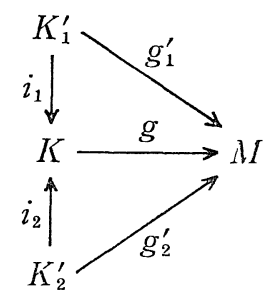

Proof. If $r \neq \omega$, the lemma coincides with Theorem 10.4, [12] except the last requirements, $g_{1}^{\prime}=g_{1}$ on $\left|L_{1}\right|, g_{2}^{\prime}=g_{2}$ on $\left|L_{2}\right|$. But these conditions are easy to see by the method of construction of $g_{1}^{\prime}, g_{2}^{\prime}$ in [12]. It follows moreover that $g_{1}^{\prime}=g_{1}$ on $\left|N\left(L_{1}, K_{1}\right)\right|, g_{2}^{\prime}=g_{2}$ on $\left|N\left(L_{2}, K_{2}\right)\right|$. We omit making sure of it.

If $r=\omega$, regard $g_{1}$ and $g_{2}$ as $C^{\infty}$ imbeddings, and let $K, i_{1}, i_{2}, K_{1}^{\prime}, K_{2}^{\prime \prime}, g_{1}^{\prime}$ and 
$g_{2}^{\prime}$ be the results in the $C^{\infty}$ case. We assume however that $g_{1}^{\prime}, g_{2}^{\prime}$ are $\varepsilon / 2$-approximations of $g_{1}, g_{2}$ respectively and that $g_{1}^{\prime}=g_{1}$ on $\left|N\left(L_{1}, K_{1}\right)\right|, g_{2}^{\prime}=g_{2}$ on $\left|N\left(L_{2}, K_{2}\right)\right|$. We want to modify $g$ to be analytic. We remark that $g$ is analytic on $i_{1}\left|N\left(L_{1}, K_{1}\right)\right| \cup i_{2}\left|N\left(L_{2}, K_{2}\right)\right|$. Let $\delta:|K| \rightarrow \mathbb{R}$ be a positive continuous function such that for any $\delta$-approximation $h: K^{\prime} \rightarrow \boldsymbol{R}^{n}$ of the zero map $0: K \rightarrow \boldsymbol{R}^{n}$, $h \circ i_{1}$ and $h \circ i_{2}$ are $\varepsilon / 2$-approximations of the zero maps $0: K_{1}^{\prime} \rightarrow \mathbb{R}^{n}$ and $0: K_{2}^{\prime} \rightarrow$ $\boldsymbol{R}^{n}$ respectively. Apply Lemma 6.10 to $g: K \rightarrow M$ and $\delta$. Let $g^{\prime}: K^{\prime} \rightarrow M$ be the resulting analytic $\delta$-approximation of $g$ such that $g=g^{\prime}$ on $i_{1}\left(\left|L_{1}\right|\right) \cup i_{2}\left(\left|L_{2}\right|\right)$. Let $K_{1}^{\prime \prime}, K_{2}^{\prime \prime}$ be the subdivisions of $K_{1}^{\prime}, K_{2}^{\prime}$ respectively such that $i_{1}$ on $K_{1}^{\prime \prime}$ and $i_{2}$ on $K_{2}^{\prime \prime}$ are linear isomorphisms onto the images. Then $K^{\prime}, i_{1}: K_{1}^{\prime \prime} \rightarrow K^{\prime}, i_{2}$ : $K_{2}^{\prime \prime} \rightarrow K^{\prime}$ and $g^{\prime}: K^{\prime} \rightarrow M$ satisfy the requirements of Lemma 6.13. Hence the lemma is proved.

Proposition 6.14. Even if $M$ has boundary, it has a $C^{\omega}$ triangulation.

Proof. Let $h: L \rightarrow \partial M, g_{1}: K_{1} \rightarrow M-\partial M$ be $C^{\omega}$ triangulations (Lemma 6.11), and $\psi: \partial M \times[0,1] \rightarrow M$ be an analytic collar such that $\phi(x, 0)=x$. Let $K_{2}$ be a simplicial subdivision of the cell complex $L \times[0,1]$. Then $g_{2}=\psi \circ(h$, ident $): K_{2}$ $\rightarrow M$ is a $C^{\omega}$ imbedding whose image is a closed collar of $M$.

Subdivide $K_{1}$ finely enough, and assume that if we put

$$
K_{3}=\left\{\sigma \in K_{1} \mid g_{1}(\sigma) \cap \phi(\partial M \times[0,1 / 3])=\varnothing\right\},
$$

we have

$$
g_{1}\left(\left|K_{3}\right|\right) \supset M-\psi(\partial M \times[0,2 / 3]) .
$$

Put $g_{3}=\left.g_{1}\right|_{K_{3}}$. Then $g_{3}: K_{3} \rightarrow M$ is a $C^{\omega}$ imbedding whose image is closed in $M$. Now we remark that Lemma 6.13 holds true even if $M$ has boundary in the case in which $g_{1}^{-1}(\partial M) \subset\left|L_{1}\right|$ and $g_{2}^{-1}(\partial M) \subset\left|L_{2}\right|$. Hence, by Lemma 6.13, we have a complex $K$, linear isomorphisms $i_{1}$ and $i_{2}$ from $K_{2}^{\prime}$ and $K_{3}^{\prime}$, subdivisions of $K_{2}$ and $K_{3}$ respectively, to subcomplexes of $K$ and a $C^{\omega}$ imbedding $g: K \rightarrow M$ such that $g_{2}^{\prime}=g \circ i_{1}, g_{3}^{\prime}=g \circ i_{2}$ are close approximations of $g_{2}, g_{3}$ respectively and that $g_{2}^{\prime}=g_{2}$ on a polyhedral neighborhood of $|L| \times 0$ in $\left|K_{2}\right|$. If the above approximations are sufficiently close, we have

$$
g_{2}^{\prime}\left(\left|K_{2}\right|\right) \supset \phi(\partial M \times[0,3 / 4])
$$

because of $g_{2}^{\prime}=g_{2}$ near $|L| \times 0$, and

$$
g_{3}^{\prime}\left(! K_{3} !\right) \supset M-\psi(\partial M \times[0,3 / 4]),
$$

namely $g$ is surjective. Hence $g$ can be a $C^{\omega}$ triangulation of $M$.

Lemma 6.15. Let $M_{1}$ be an analytic manifold of dimension $=\operatorname{dim} M-1$ possibly with boundary, let $\phi: M_{1} \times[0,1] \rightarrow M$ be an analytic imbedding whose inage is closed in $M$, let $h: L \rightarrow M_{1}$ be a $C^{\omega}$ triangulation, let $K_{1}$ be a simplicial subdivision of the cell complex $L \times[0,1]$, and let $K_{2}$ be a subcomplex of $K_{1}$ whose image 
under (h, ident) does not intersect with $\partial\left(M_{1} \times[0,1]\right)$. Put

$$
g_{2}=\left.\phi \circ(h, i d e n t)\right|_{K_{2}} .
$$

Then there exists a $C^{\omega}$ triangulation $g: K \rightarrow M$ and a subcomplex $K_{3}$ of $K$ such that

$$
g\left(\left|K_{3}\right|\right)=g_{2}\left(\left|K_{2}\right|\right)
$$

and that $g_{2}^{-1} \circ \mathrm{g}: K_{3} \rightarrow K_{2}$ is a linear isomorphism.

The proof proceeds just in the same way as Proposition 6.14, so we omit it.

We will apply Lemma 6.15 to the proof of Theorem I later in the following situation. The analytic function $f$ is $C^{\infty}$ regular on $\phi\left(M_{1} \times[0,1]\right)$, and for any connected component $C$ of $M_{1}$ there is a constant $c$ such that $f \circ \psi(x, t)=t+c$, $(x, t) \in C \times[0,1]$. Then $f \circ g$ is subanalytic on $|K|$ and $P L$ on $\left|K_{3}\right|$, namely $f$ can be piecewise linearized on an arbitrarily large subset of Int $\phi\left(M_{1} \times[0,1]\right)$ closed in $M$.

Corollary 6.16. Let $g: K \rightarrow M$ be a $C^{\omega}$ triangulation. Given a locally finite family $\left\{X_{i}\right\}$ of subanalytic subsets of $M$, there exist a subdivision $K^{\prime}$ of $K$ and a subanalytic homeomorphism $\tau:|K| \rightarrow M$ such that for any $\sigma \in K, \tau(\sigma)=g(\sigma)$, that for any $\sigma \in K^{\prime}, \tau\left(\dot{\sigma}^{\circ}\right)$ is an analytic submanifold of $M$ and $\left.\tau\right|_{\dot{\sigma}}: \stackrel{\circ}{\sigma} \rightarrow \tau(\stackrel{\circ}{\sigma})$ is an analytic diffeomorphism and that $\left\{\tau(\stackrel{\circ}{)}) \mid \sigma \in K^{\prime}\right\}$ is compatible with $\left\{X_{i}\right\}$.

Proof. Assume $K$ is contained in $\boldsymbol{R}^{m}$ so that $|K|$ is closed in $\boldsymbol{R}^{m}$. Since $g$ is of class $C^{\omega}$, by (2.5.1) $\left\{g^{-1}\left(X_{i}\right)\right\}$ is a locally finite family of subanalytic subsets of $\boldsymbol{R}^{m}$. Hence the corollary follows from Proposition 3.1'.

Let us consider the semi-algebraic case of the above results. The proofs of the results below proceed in the same way as the $C^{\omega}$ case, so we omit the details of proofs.

Definition 6.17. If $M$ is semi-algebraic in $\boldsymbol{R}^{n}$, we call it a Nash manifold. An analytic map between Nash manifolds is called a Nash map if the graph is semi-algebraic. A $C^{\omega}$ map $g: K \rightarrow M, K$ being finite, is called of class Nash if the graph is semi-algebraic.

If $K$ is finite, Lemma 6.8 holds true in the case of Nash map too since $\rho$ and $\alpha$ in the proof of Lemma 6.8 are of class Nash. In Lemmas 6.9, 10, if $M$ is a Nash manifold, if $K$ is finite and if $\left.g\right|_{\left|K_{2}\right|}$ is of class Nash, then $\left.g^{\prime}\right|_{\left|K_{1} \cup K_{2}\right|}$ in Lemma 6.9 and $g^{\prime}$ in Lemma 6.10 can be of class Nash. We remark here that any secant map on a finite complex is of class Nash. We obtain also the Nash case of Lemma 6.13. As the replacement is clear, we omit the details. By these facts, we have the following Nash case of Propositions 6.11, 14 .

Proposition 6.18. If $M$ is a compact Nash manifold possibly with boundary, then it has a Nash triangulation, namely, there exist a finite complex $K$ and $a$ 
triangulation $g: K \rightarrow M$ of class Nash.

For the case of non-compact Nash manifolds, we need the following

Lemma 6.19 (Theorem 1, [17]). If $M$ is a non-compact Nash manifold, there exists a compact Nash manifold $M^{\prime}$ with boundary such that $\mathrm{I}$ is Vash diffeomorphic to $M^{\prime}-\partial M^{\prime}$.

Lemma 6.20. Let $M$ be a non-compact Nash manifold. Then there exist a

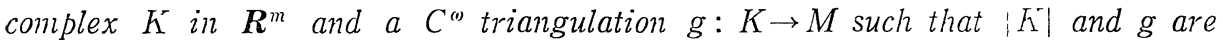
semi-algebraic.

Proof. Apply Proposition 6.18 to $M^{\prime}$ in Lemma 6.19. Let $\mathrm{g}^{\prime}: K^{\prime} \rightarrow M^{\prime}$, $K^{\prime} \subset \boldsymbol{R}^{m}$, be a Nash triangulation. Then $\left|K^{\prime}\right|$ is a $P L$ manifold with boundary (Remark 6.12). Hence we have $g^{\prime}\left(\partial\left|K^{\prime}\right|\right)=\partial M^{\prime}$, so a triangulation $K^{\prime}$ of $\left|K^{\prime}\right|$ $\partial\left|K^{\prime}\right|$ compatible with $K^{\prime \prime}$ and $g=\left.g^{\prime}\right|_{\left|K^{\prime}\right|}$ satisfy the requirement of Lemma 6.20.

Corollary 6.21. Let $M$ be a compact Nash manifold possibly uith boundary, and let $g: K \rightarrow M$ be a Nash triangulation. Given a finite family $\left\{Y_{i}\right\}$ of semialgebraic subsets of $M$, there exist a subdivision $K^{\prime}$ of $K$ and a semi-algebraic homeomorphism $\tau:|K| \rightarrow M$ such that $\tau(\sigma)=g(\sigma)$ for any $\sigma \subseteq K$, that for any $\sigma \in K^{\prime}, \tau(\dot{\sigma})$ is a Nash submanifold of $M$ and $\left.\tau\right|_{\circ}: \stackrel{\circ}{\sigma} \rightarrow \tau(\stackrel{\circ}{\sigma})$ is a lash diffeomorphism and that $\left\{\tau(\dot{\sigma}) \mid \sigma \in K^{\prime}\right\}$ is compatible with $\left\{X_{\imath}\right\}$.

Proof. Clear by Proposition 3.9.

Corollary 6.22. Let $M$ be a non-compact Nash manifoid. Gnen a finitc family $\left\{X_{\imath}\right\}$ of semi-algebraic subsets of $M$, there exist a complex $K$ in $\boldsymbol{R}^{m}$, a $C^{\omega}$ triangulation $g: K \rightarrow M$ and a homeomorphism $\tau:|K| \rightarrow . M$ such that $|K|$ and $\tau$ are semi-algebraic, that for any $\sigma \in K, \tau(\stackrel{\circ}{\sigma})$ is a Nash submanifuld of $M$ and $\left.\tau\right|_{\dot{\sigma}}: \hat{\sigma} \rightarrow \tau(\hat{\sigma})$ is a Nash diffeomorphism and that $\{\tau(\dot{\sigma}) \mid \sigma \in K\}$ is compatible with $\left\{X_{l}\right\}$.

Proof. We only need to remark that for a compact Nash manifold $M^{\prime}$ with boundary, any semi-algebraic subset of $M^{\prime}-\partial M^{\prime}$ is also semi-algebraic in $M^{\prime}$.

Corollaries 6.21, 22 are generalizations of Theorem 3, [10] which treated only the case $M=\boldsymbol{R}^{n}$.

Remark 6.23. $|K|$ in Corollaries $6.16,21,22$ are $P L$ manifolds by Remark 6.12. If it were not so, we could not apply Concordance Implies Isotopy Theorem to the proof of Theorem I. This is one of the reasons why we refined a result of [4] in Proposition 3.1. It is difficult to obtain the corollaries br the result of $[4]$. 


\section{§7. Proof of Theorem I}

The last but not least tool of the proof is Concordance Implies Isotopy Theorem of Kirby-Siebenmann [6].

Let $P, Q$ be polyhedrons. A map $f: P \rightarrow \boldsymbol{R}^{n}$ is called a $P L$ map if there is a simplicial complex $K$ with $|K|=P$ such that $f$ is linear on each simplex of $K$. A map $f: P \rightarrow Q$ is called a $P L$ map if $Q$ is $P L$ imbedded in some $R^{m}$ so that $f: P \rightarrow \boldsymbol{R}^{m}$ is a $P L$ map. A homotopy $\phi_{t}, 0 \leqq t \leqq 1$, between topological spaces is called an isotopy if for each $t, \psi_{t}$ is a homeomorphism.

Lemma 7.1 ([6]). Let $M_{1}, M_{2}$ be metrized $P L$ manifolds, $\phi: M_{1} \times[0,1] \rightarrow$ $M_{2}$ be a homeomorphism, $C \subset M_{1}$ be a closed subset, and $\varepsilon$ be a positive continuous function on $M_{1} \times[0,1]$. Assume that $\operatorname{dim} M_{2} \neq 4,5$ and that $\psi$ is $P L$ on a neighborhood of $M_{1} \times 0 \cup C \times[0,1]$. Then there exists an isotopy $\psi_{t}: M_{1} \times[0,1] \rightarrow M_{2}$, $0 \leqq t \leqq 1$, of $\phi$ such that

(7.1.1) $\phi_{1}$ is a PL homeomorphism,

(7.1.2) $\psi_{t}=\phi, 0 \leqq t \leqq 1$, on a neighborhood of $M_{1} \times 0 \cup C \times[0,1]$, and that

(7.1.3) $\operatorname{dist}\left(\phi_{t}(x), \phi(x)\right)<\varepsilon(x)$ for all $x \in M_{1} \times[0,1], 0 \leqq t \leqq 1$.

Now we have finished preparing for the proof of Theorem I. We begin to prove it. The proof consists of seven steps (7.2), , , (7.8).

(7.2) We assume $M$ to be non-compact, because the compact case is easier to prove. There exist compact analytic submanifolds with boundary $M_{1}, M_{2}, \cdots$ and without boundary $N_{0}=\varnothing, N_{1}, N_{2}, \cdots$ of $M$ such that

$$
\begin{aligned}
& \bigcup_{i=1}^{\infty} M_{\imath}=M, \quad \partial M_{i}=N_{\imath-1} \cup N_{i}, \\
& M_{\imath} \cap M_{\imath+1}=N_{i}, M_{i} \cap M_{\jmath}=\varnothing \text { for any } i, j \text { with }|i-j| \geqq 2 .
\end{aligned}
$$

They are constructed for example as follows. Assume $M$ is closed in $\boldsymbol{R}^{n}$. Put $\varphi(x)=|x|^{2}, x \in M$. Then $\varphi$ is a positive proper analytic function on $M$. Let $a_{0}=-1<a_{1}<a_{2}<\cdots$ be a sequence of $C^{\infty}$ regular values of $\varphi$ tending to infinity such that $\varphi(M) \ni a_{i}$. Then

$$
M_{\imath}=\varphi^{-1}\left(\left[a_{i-1}, a_{i}\right]\right) \text { and } N_{i}=\varphi^{-1}\left(a_{\imath}\right), i=1,2, \cdots
$$

satisfy the conditions above.

Put $N=\bigcup_{i=1}^{\infty} N_{i}$. Then $N$ is an analytic manifold closed in $M$. We remark the fact that the set of critical values of an analytic function on a compact manifold (may having boundary) is a finite set. Let $S_{1}, S_{2}, \cdots$ be a sequence of finite subsets of $R$ such that for each $i, S_{i}$ contains all critical values of $\left.f\right|_{M_{i}}$, $\left.f\right|_{N_{i-1}}$ and $\left.f\right|_{N_{i}}$ and that $S_{i} \supset S_{i-1} \cup S_{i+1}$ for even $i$. We put $S_{0}=\varnothing$ and $M_{0}=\varnothing$ for convenience. Put 


$$
\begin{aligned}
& Y=\bigcup_{i=1}^{\infty}\left(M_{\imath} \cap f^{-1}\left(S_{\imath}\right)\right), \\
& Y_{1}=\bigcup_{i=1}^{\infty} N_{\imath} \cap f^{-1}\left(\left(S_{\imath}-S_{i+1}\right) \cup\left(S_{\imath+1}-S_{\imath}\right)\right) \\
& Y_{2}=X \cap N-Y_{1}=\bigcup_{i=1}^{\infty} N_{i} \cap f^{-1}\left(S_{i} \cap S_{i+1}\right) .
\end{aligned}
$$

Then $X, Y_{1}$ and $Y_{2}$ are a closed semi-analytic set and analytic sets respectively. We remark that $X$ and $Y_{2}$ contains all critical points of $f$ and $\left.f\right|_{.}$respectively.

(7.3) Applying Lemma 6.15, we want to piecewise linearize $f$ on a large domain containing $Y_{1}$. There exist an open neighborhood $U$ of $Y_{1} \cup(M-X)$ in $M$ and a $C^{\infty}$ vector field $\xi$ on $U$ such that $\xi f>0$ on $U$ and that $\left.\xi\right|_{\nu \cap U}$ is a vector field on $N \cap U$. Indeed, put $\xi_{1}=d f$, and let $\xi_{2}$ be an extension of $d\left(\left.f\right|_{N}\right)$ to a $C^{\infty}$ vector field on $M$. Then we have an open neighborhood $U_{1}$ of $N-Y_{2}$ in $M$ such that $\xi_{1} f>0$ on $U_{1} \cup(M-X)$ and $\xi_{2} f>0$ on $U_{1}$. Let $U_{2}$ be another neighborhood of $N-Y_{2}$ with $\bar{U}_{2}-U_{1} \subset Y_{2}$, and let $\rho$ be a $C^{\infty}$ function on $M-Y_{2}$ such that $0 \leqq \rho \leqq 1, \rho=0$ on $U_{2}$ and $=1$ outside $U_{1}$. Then $\xi=\rho \xi_{1} \div(1-\rho) \xi_{2}$ and $U=$ $U_{1} \cup(M-X)$ satisfy the required conditions.

Multiplying $\xi$ by $1 / \xi f$, we assume moreover $\xi f=1$ on $U$. Let $\Theta: D \rightarrow U$ be the $C^{\infty}$ flow of $\xi$, namely, $\Theta$ is defined by

$$
\Theta(x, 0)=x, \frac{\partial}{\partial t} \Theta(x, t)=\xi_{\Theta(x, t)}
$$

and $D \subset U>\boldsymbol{R}$ is the maximal open set containing $U \times 0$. Then we have

(7.3.1) $f \circ \Theta(x, t)=f(x)+t \quad$ for $(x, t) \in D$ because of $\xi f=1$,

(7.3.2) $\Theta(x, t) \in N \quad$ for $x \in N,(x, t) \in D$.

(7.3.3) $\Theta(x, t) \in M_{2} \quad$ for $x \in M_{i},(x, t) \in D, i=1, \cdots$.

Let $i$ be a positive odd integer. Let $S_{\imath}^{0}$ be the open $\delta_{\imath}$-neighborhood of $S_{\imath}$ in $R$ for some small $\delta_{i}>0$ such that

(7.3.4) $\bar{S}_{i}^{0} \cap\left(S_{\iota+1} \cup S_{i-1}\right)=S_{i}$.

Put

$$
\begin{aligned}
& S_{\imath}^{+}=S_{\imath}+\hat{o}_{\imath}=\left\{t+\hat{o}_{\imath} \mid t \in S_{\imath}\right\} \quad \text { and } \\
& S_{\imath}^{-}=S_{\imath}-\delta_{\imath}=\left\{t-\delta_{i} \mid t \in S_{\imath}\right\} .
\end{aligned}
$$

Then $f^{-1}\left(S_{i}^{-}\right) \cap M_{i}$ and $f^{-1}\left(S_{i}^{-}\right) \cap M_{i}$ are compact analytic manifolds with boundary in $N_{i-1} \cup N_{i}$. Under $\Theta, f^{-1}\left(\boldsymbol{R}-S_{2}^{0}\right) \cap M_{i}$ is $C^{\infty}$ diffeomorphic to $\left(f^{-1}\left(S_{\imath}^{+}\right) \cap M_{i}\right) \times$ $[0,1]$. We will enlarge $f^{-1}\left(\boldsymbol{R}-S_{2}^{0}\right)$ a little, and it is on this set that we will piecewise linearize $f$ in (7.3). 
Put

$$
\begin{aligned}
& L_{i}=f^{-1}\left(S_{i}^{+}\right) \cap \varphi^{-1}\left(\left[a_{i-1}-\delta_{i}^{\prime}, a_{\imath}+\delta_{i}^{\prime}\right]\right), \\
& L_{\imath}^{\prime}=f^{-1}\left(S_{i}^{+}\right) \cap \varphi^{-1}\left(\left[a_{i-1}-\delta_{i}^{\prime} / 2, a_{i}+\delta_{\imath}^{\prime} / 2\right]\right)
\end{aligned}
$$

for small $\delta_{2}^{\prime}>0$ where $\varphi$ is defined in (7.2). Choose $\delta_{i}^{\prime}$ small enough. Then $L_{i}$, $L_{\imath}^{\prime}$ are compact analytic manifolds with boundary such that $L_{i}-\partial L_{\imath} \supset L_{\imath}^{\prime}, L_{i}^{\prime}-$ $\partial L_{i}^{\prime} \supset f^{-1}\left(S_{i}^{+}\right),-. M_{2}$ and that $(x, t) \in D$ for $x \in L_{i}, 0 \leqq t \leqq \min \left\{s \in S_{i}^{-} \mid s>f(x)\right\}-f(x)$. It means that we have a $C^{\infty}$ imbedding $\phi_{i}: L_{i} \times[0,1] \rightarrow U$ such that $\phi_{i}(x, 0)=$ $x, f \circ \phi_{i}(x, 1) \equiv S_{\imath}^{-}$for $x \in L_{i}$, that for each $x \in L_{i}, \phi_{\imath}(x \times[0,1])$ is contained in the integral curse of $\xi$ through $x$ and that if $\Theta\left(x, t_{0}\right)=\phi_{i}(x, 1), t_{0} \in \boldsymbol{R}$ then $\psi_{i}(x, t)=\Theta\left(x, t_{0} t\right), 0 \leqq t \leqq 1$. The last property together with (7.3.1) tells us that $\left.f \circ \zeta\right|_{x \times[0,1]}$ is linear for each fixed $x$.

From the other properties and $(7.3 .3,4)$ it follows that for each connected component $C$ of $L_{\imath}, C \times \boldsymbol{R} \cap D \subset C \times\left(s_{1}-f(C), s_{2}-f(C)\right)$ where $\left(s_{1}, s_{2}\right)$ is the connected component of $\boldsymbol{R}-S_{i}$ containing $f(C)$. We see easily from (7.3.2) that the set $\Theta\left(C\right.$ 以 $\left.\left\{s_{1}-f(C), s_{2}-f(C)\right\} \cap D\right)$ has a positive distance from $N_{i-1} \cup N_{i+1}$ if it is not empty. Hence, shrinking $U$ near $f^{-1}\left(\left\{s_{1}, s_{2}\right\}\right) \cap\left(N_{i-1} \cup N_{i+1}\right)$ if necessary, we can assume $C \times\left\{s_{1}-f(C), s_{2}-f(C)\right\} \cap D=\varnothing$. Then we have

$$
C \times \boldsymbol{R} \cap D=C \times\left(s_{1}-f(C), s_{2}-f(C)\right),
$$

and hence $\left.\Theta\right|_{L \quad R \rightarrow D}: L \times \boldsymbol{R} \cap D \rightarrow M$ is a $C^{\infty}$ imbedding. Choose $\delta_{j}^{\prime}$ for all odd $j$ so small that

$$
\Theta\left(L_{j^{\prime}}: \boldsymbol{R}_{\cap} \cap D\right) \cap \Theta\left(L_{j^{\prime \prime}} \times \boldsymbol{R} \cap D\right)=\varnothing \quad \text { for odd } j^{\prime} \neq j^{\prime \prime} .
$$

Before applying Lemma 6.15 , we need to modify $\xi$ to be analytic. Remember $M \subset \mathbb{R}^{n}$. Let us regard $\xi_{x}$ as a tangent vector of $\boldsymbol{R}^{n}$ at $x$, namely $\xi$ is regarded as a $C^{\infty}$ map from $U$ to $\boldsymbol{R}^{n}$. Approximate $\xi$ by an analytic map $\xi^{\prime}$ in the Whitney topology [5]. Let $p_{x}, x \in M$, be the orthogonal projection of the tangent space $T_{x} R^{n}$ to $T_{x} M$. Then $\left\{p_{x} \circ \xi_{x}^{\prime} \mid x \in M\right\}$ is an analytic vector field on $M$ close to $\xi$. We use the same notation $\xi$ for the approximation. Choose the approximation so close that $\xi f>0$ on $U$. Assume $\xi f=1$ on $U$ by the same reason as before. We then define the flow of new $\xi$ too and use the same notation $\Theta: D \rightarrow U$ for it. Consider the conditions (7.3.1, $\cdots, 6)$. Clearly (7.3.1) remains true, and (7.3.6) can do so for some smaller $\delta_{\imath}^{\prime}$, but $(7.3 .2,3)$ fail in general, (the fact is, we can continue them by using the analytic sheaf theory in the same way as Chapter II, [16]). We assume in place of $(7.3 .2,3)$ that

$$
\begin{aligned}
& \left.\Theta i\left(L_{j}^{\prime}-\partial L_{j}^{\prime}\right) \times \boldsymbol{R} \cap D\right) \supset M_{\jmath}-f^{-1}\left(S_{\jmath}\right) \quad \text { for any odd } j, \text { and } \\
& \left.\Theta_{(}^{\prime} L_{\imath}: \boldsymbol{R} \cap D\right) \subset M_{i} \cup\left(M_{i-1}-\partial M_{\imath-1}\right) \cup\left(M_{i+1}-\partial M_{i+1}\right)
\end{aligned}
$$

which follows from $(7.3 .6,7)$ for $j=i-2, i$ and $i+2$. The assumptions are clearly possible if the approximation is close enough. By (7.3.8) and by the same reason 
as before, (7.3.5) can remain true. We remark that $\Theta$ and $\phi_{i}$ are analytic and that the image of $\phi_{i}$ is closed in $M$.

Put

$$
L=\bigcup_{i: \text { odd }} L_{i}, \quad L^{\prime}=\bigcup_{i: \text { odd }} L_{\imath}^{\prime},
$$

and let $\phi: L \times[0,1] \rightarrow M$ be defined by $\left.\phi\right|_{L_{i} \times[0,1]}=\psi_{i}$. By Proposition 6.14, we have a $C^{\omega}$ triangulation $h: W \rightarrow L$. Subdivide $W$ finely enough. Then we have a subcomplex $W^{\prime \prime}$ of $W$ whose underlying polyhedron is a $P L$ manifold with boundary, is contained in Int $|W|$ and contains $h^{-1}\left(L^{\prime}\right)$ in its interior (see Chapter 2, 3, [13]). Put $L^{\prime \prime}=h\left(\left|W^{\prime \prime}\right|\right)$. Let $\tilde{\phi}_{i}: L_{i} \times\left[-\delta_{i}^{\prime \prime}, 1+\delta_{i}^{\prime \prime}\right] \rightarrow M$ be a welldefined analytic extension of $\phi_{i}$ which is an imbedding for small $\delta_{i}^{\prime \prime}>0$. Apply Lemma 6.15 to $\left\{\tilde{\psi}_{i}\right\}_{i \text { : odd }}$ and $h: W \rightarrow L$. Then we have a $C^{\omega}$ triangulation $g$ : $K \rightarrow M$ and a subcomplex $K_{1}$ of $K$ such that

$$
\begin{gathered}
g\left(\left|K_{1}\right|\right)=\psi\left(L^{\prime \prime} \times[0,1]\right), \text { and that } \\
(h, \text { ident })^{-1} \circ \psi^{-1} \circ g:\left|K_{1}\right| \longrightarrow\left|W^{\prime \prime}\right| \times[0,1]
\end{gathered}
$$

is a $P L$ homeomorphism. Hence we can assume moreover that a subcomplex $K_{2}$ of $K_{1}$ has the underlying polyhedron which is carried by $g$ onto $\phi\left(L^{\prime \prime} \times 0\right)$. Then

$$
(h, \text { ident })^{-1} \circ \psi^{-1} \circ g:\left|K_{2}\right| \longrightarrow\left|W^{\prime \prime}\right| \times 0
$$

is a $P L$ homeomorphism. Now, by the definition of $\psi, f \circ \psi \circ(h$, ident $)$ is a $P L$ function on $|W| \times[0,1]$. Hence (7.3.9) tells us that

$$
f \circ g \text { is } P L \text { on }\left|K_{1}\right| \text {. }
$$

We can assume that $g: K \rightarrow M$ given in Theorem I coincides with the above $g: K \rightarrow M$. The reason is the following. Let $g^{*}: K^{*} \rightarrow M$ be another $C^{\infty}$ triangulation. Then by Remark 6.12 there are $C^{\infty}$ approximations $g^{\prime}: K^{\prime} \rightarrow M, g^{* \prime}$ : $K^{* \prime} \rightarrow M$ of $g, g^{*}$ respectively such that $g^{\prime-1} \circ g^{* \prime}$ is a linear isomorphism. Hence $g \circ g^{\prime-1} \circ g^{* \prime}: K^{* \prime} \rightarrow M$ is an approximation of $g^{*}$. As $g^{* \prime-1} \circ g^{\prime}\left(\left|K_{1}\right|\right)$ is the underlying polyhedron of a subcomplex $K_{1}^{* \prime}$ of $K^{* \prime}$, and since $f \circ g$ is $P L$ on $\left|K_{1}\right|$, $f \circ g \circ g^{\prime-1} \circ g^{* \prime}$ is $P L$ on $\left|K_{1}^{* \prime}\right|$. Hence we can replace $g: K \rightarrow M, K_{1}$ by $g \circ g^{\prime-1} \circ$ $g^{* \prime}: K^{* \prime} \rightarrow M, K_{1}^{* \prime}$ respectively. Therefore the assumption above is admitted.

(7.4) For any subset $A$ of $M$, let $A^{*}$ denote the inverse image of $A$ under $g:|K| \rightarrow M$. Put

$$
D^{*}=\left\{(x, t) \in U^{*} \times \boldsymbol{R} \mid(g(x), t) \in D\right\} .
$$

Let $\Theta^{*}: D^{*} \rightarrow U^{*}, \psi^{*}: L^{*} \times[0,1] \rightarrow U^{*}, h^{*}: W \rightarrow L^{*}, f^{*}: M^{*} \rightarrow \boldsymbol{R}$ and $\phi_{i}^{*}: L_{i}^{*} \times$ $[0,1] \rightarrow M^{*}, i$ : odd, be defined by $\Theta^{*}=g^{-1} \circ \Theta \circ(g$, ident $), \psi^{*}=g^{-1} \circ \% \circ(g$, ident $)$, $h^{*}=g^{-1} \circ h, f^{*}=f \circ g$ and $\phi_{i}^{*}=g^{-1} \circ \psi_{i} \circ(g$, ident $)$. From now on we consider $f^{*}$ on $M^{*}$ in place of $f$ on $M$ and ident $: K \rightarrow M^{*}$ in place of $g: K \rightarrow M$ in Theorem 
I. We summarize what we obtained.

(7.4.1) $M^{*}, L^{\prime \prime *}$ are a $P L$ manifold and one with boundary respectively by Remark 6.12 and (7.3.10). $L^{\prime *}, X^{*}, Y_{1}^{*}$, and $Y_{2}^{*}$ are closed subanalytic subsets of $M^{*}$. We have

Put

$$
|K|=M^{*},\left|K_{2}\right|=L^{\prime \prime *}=\phi^{*}\left(L^{\prime \prime *} \times 0\right),\left|K_{1}\right|=\phi^{*}\left(L^{\prime \prime *} \times[0,1]\right) 。
$$

$$
\left|K_{1}\right|_{\imath}=\phi_{i}^{*}\left(L_{i}^{\prime \prime *} \times[0,1]\right), \quad i=1,3, \cdots .
$$

(7.4.2) $\Theta^{*}, \phi^{*}, h^{*}$ are subanalytic maps. $f^{*}$ on $X^{*}$ is locally constant.

(7.4.3) $f^{*} \circ \Theta^{*}(x, t)=f^{*}(x)+t$ for $(x, t) \in D^{*}$ by (7.3.1).

(7.4.4) For any connected component $C$ of $L_{i}^{*}, i$ : odd,

$$
C \times \boldsymbol{R} \cap D^{*}=C \times\left(s_{1}-f^{*}(C), s_{2}-f^{*}(C)\right)
$$

where $\left(s_{1}, s_{2}\right)$ is the connected component of $\boldsymbol{R}-S_{i}$ containing $f^{*}(C)$ by (7.3.5).

$$
\left.\phi^{*}\right|_{L^{p *} \times[0,1]}: L^{\prime \prime *} \times[0,1] \longrightarrow\left|K_{1}\right|
$$

is a $P L$ homeomorphism by (7.3.9) and because $\left.h^{*}\right|_{\left|W^{\prime \prime}\right|}:\left|W^{\prime \prime}\right| \rightarrow L^{\prime \prime *}$ is a $P L$ homeomorphism by (7.3.10).

(7.4.6) The definition of $\psi$ shows that

$$
\begin{aligned}
& \psi^{*}(x, 0)=x \quad \text { for } \quad x \in L^{\prime \prime *}, \\
& f^{*} \circ \psi_{i}^{*}(x, 1) \in S_{i}^{-} \quad \text { for each odd } i \text { and } x \in L_{\imath}^{\prime \prime *}, \\
& \psi^{*}(x \times[0,1]) \subset \Theta^{*}(x \times R \cap D) \quad \text { for each } \quad x \in L^{\prime \prime *}, \text { and }
\end{aligned}
$$

$\left.f^{*} \circ \psi^{*}\right|_{x \text { [0,1] }}$ is linear for each $x \in L^{\prime \prime *}$.

(7.4.7) $\left.f^{*}\right|_{\left|K_{1}\right|}$ is $P L$ by (7.3.11).

(7.4.8) $\quad \Theta^{*}\left(\left(L_{i}^{\prime *}-\partial L_{i}^{\prime *}\right) \times \boldsymbol{R} \cap D^{*}\right) \supset M_{i}^{*}-f^{*-1}\left(S_{i}\right), i$ : odd, by (7.3.7).

(7.4.9) $\quad \Theta^{*}\left(L_{\imath}^{*} \times \boldsymbol{R} \cap D^{*}\right) \subset M_{\imath}^{*} \cup\left(M_{\imath-1}^{*}-\partial M_{i-1}^{*}\right) \cup\left(M_{i+1}^{*}-\partial M_{i+1}^{*}\right), \quad i$ : odd,

$$
\text { by (7.3.8) . }
$$

(7.4.10) $\Theta^{*}$ is one-to-one on $L^{\prime \prime *} \times \boldsymbol{R} \cap D$ by $(7.3 .5,6)$ and $P L$ on $L^{\prime \prime *} \times \boldsymbol{R} \cap \Theta^{*-1}$ $\left(\left|K_{1}\right|\right)$ by $(7.4 .5,6)$.

The statement that $\Theta^{*}$ is $P L$ and one-to-one on $L^{\prime \prime *} \times \boldsymbol{R} \cap \Theta^{*-1}\left(\left|K_{1}\right|\right)$ is equivalent to

(7.4.10) $\Theta^{\prime}$ is a $P L$ homeomorphism from $\Theta *(C, c) \times\left[-c, s_{2}-s_{1}-2 \delta_{i}-c\right]$ to the connected component of $\left|K_{1}\right|$ containing $C$ for any connected component $C$ of $L_{\imath}^{\prime \prime *}$ and any $0 \leqq c \leqq s_{2}-s_{1}-2 \delta_{i}$ where $\left(s_{1}, s_{2}\right)$ is defined in (7.4.4).

(7.5) Here we triangulate $X^{*}$. For each odd $i>0, Z_{i}=\left|K_{1}\right|_{i} \cap f^{*-1}\left(\left(S_{i+1} \cup\right.\right.$ $\left.S_{2-1}\right)-S_{i}$ ) is a polyhedron by (7.4.7) and contains $X^{*} \cap\left|K_{1}\right|_{i}$ by the definition 
of $X, S_{\imath}, S_{\imath+1}$ and $S_{i-1}$. Put $Z=\bigcup_{i \text { odd }} Z_{i}$. Apply Proposition $3.1^{\prime}$ to $K$ and $X^{*}$ $\cup Z$. Then we have a subdivision $K^{\prime}$ of $K$ and a subanalytic homeomorphism $\tau_{1}$ of $M^{*}$ such that $\left\{\tau_{1}(\stackrel{\circ}{\sigma}) \mid \sigma \in K^{\prime}\right\}$ is compatible with $X^{*} \cup Z$. Since $X^{*}$ and $Z$ are closed in $M$, it follows that $\tau_{1}^{-1}\left(X^{*} \cup Z\right)$ is a subpolyhedron of $M^{*}$. Moreover Remark 3.8 tells us that $\tau_{1}$ can be the identity outside an arbitrarily small neighborhood of $\overline{X^{*}-Z}=\overline{X^{*}-\left|K_{1}\right|}$ since $Z$ is a subpolyhedron and since $X^{*} \cup Z$ coincides with $Z$ outside $\overline{X^{*}-Z}$. Hence the properties in (7.4) remain true when we shrink $\left|K_{2}\right|$ and $\left|K_{1}\right|$ a little and when we shift the problem by $\tau_{1}^{-1}$, namely, when we consider $\tau_{1}^{-1}\left(X^{*}\right), f^{*} \circ \tau_{1}$, etc. in place of $X^{*}, f^{*}$, etc., so we can add the following to the properties in (7.4).

(7.5.1) $\quad X^{*} \cup Z$ is a subpolyhedron of $M^{*}$.

Since $\tau$ in Theorem $I$ is required to be a $C^{0} \varepsilon$-approximation of $g$ (=ident), for the admission of replacement of $X^{*}, f^{*}$, etc. by $\tau_{1}^{-1}\left(X^{*}\right), f^{*} \circ \tau_{1}$, etc., $\tau_{1}$ must be arbitrarily close to the identity in the $C^{0}$ fine topology. To be exact, let $M^{*} \subset \boldsymbol{R}^{n *}$, and $\varepsilon^{\prime}$ be a positive continuous function on $M^{*}$. Then $\tau_{1}$ must be chosen so that $\left|\tau_{1}(x)-x\right|<\varepsilon^{\prime}(x)$. It is possible by (3.1.2) if we subdivide $K$ so that the diameter of each $\sigma \in K$ is smaller than any $\varepsilon^{\prime}(x), x \in \sigma$.

(7.6) We want to piecewise linearize $f^{*}$ on a neighborhood of $X^{*}$. If $f^{-1} f(x) \equiv X^{*}$ as germs at $x$ for any $x \in X^{*}$, then it would follow directly from Corollary 5.12 and (7.5.1). But the equality above is not correct in general, so we remove subsets from $M^{*}$ and $X^{*}$. Let $P_{2} \subset L^{\prime \prime *}-\partial L^{\prime \prime *}$ be a $P L$ manifold with boundary closed in $L^{\prime \prime *}$ and containing $L^{\prime *}$ in its interior. Put $P_{1}=$ $\psi^{*}\left(P_{2} \times[0,1]\right)$. Then $P_{1}$ is a $P L$ manifold with boundary by $(7.4 .5), f^{*}$ is $P L$ on a neighborhood of $P_{1} \cap X^{*}$ by (7.4.7), and it also follows from the definitions of $S_{i}, L_{i}^{\prime}$ that $\left.f^{-1}\right|_{X^{*}-\operatorname{Int} P_{1}}(f(x)) \equiv X^{*}-\operatorname{Int} P_{1}$ as germs at $x$ for any $x \in X^{*}-\operatorname{Int} P_{1}$. Apply Corollary 5.12 to $M^{*}$-Int $P_{1}, X^{*}$-Int $P_{1}$ and $f^{*}$. Then we have a homeomorphism $\tau_{2}$ of $M^{*}$ - Int $P_{1}$ such that $f^{*}{ }^{*} \tau_{2}$ is $P L$ on a neighborhood of $X^{*}-\operatorname{Int} P_{1}$ in $M^{*}$ - Int $P_{1}$ and that $\tau_{2}$ is the identity on $\partial P_{1} \cup\left(X^{*}-\operatorname{Int} P_{1}\right)$ and outside a small neighborhood of $X^{*}-\operatorname{Int} P_{1}$. Extend $\tau_{2}$ to $M^{*}$ by putting $\tau_{2}=$ ident on $P_{1}$. Consider $f^{*} \circ \tau_{2}, P_{1}$, etc. in place of $f^{*}, L^{\prime \prime *}$, etc. respectively, and use the former notations for them. Then we have

(7.6.1) $f^{*}$ is $P L$ on a neighborhood of $X^{*}$.

The replacement here is admitted because $\tau_{2}$ can be arbitrarily close to the identity in the $C^{0}$ fine topology by Remark 5.13. We remark that the first half of (7.4.2) fails now and that $\Theta^{*}, \psi^{*}, h^{*}$ are only a continuous flow, continuous maps respectively. The other properties in (7.4) and (7.5.1) remain true.

(7.7) We will piecewise linearize $f^{*}$ on $\Theta^{*}\left(\left(L^{* \prime \prime}-\partial L^{\prime \prime}\right) \times \boldsymbol{R} \cap D^{*}\right)$. Let $C$ be a connected component of $L^{\prime \prime *}-\partial L^{\prime \prime *}$ contained in some $L_{\imath}^{\prime \prime *}$. Put $Q=\Theta^{*}(C \times$ $\left.\boldsymbol{R} \cap D^{*}\right)$. Let $\left(s_{1}, s_{2}\right)$ be the connected component of $\boldsymbol{R}-S_{i}$ containing $f^{*}(C)$. 
Then $C \times \boldsymbol{R} \cap D^{*}=C \times\left(s_{1}-f^{*}(C), s_{2}-f^{*}(C)\right)$ by (7.4.4). Hence we have

$$
f^{*}(Q)=\left(s_{1}, s_{2}\right), f^{*} \circ \psi_{2}^{*}(C \times 0)=s_{1}+\delta_{i}, f^{*} \circ \psi_{i}^{*}(C \times 1)=s_{2}-\delta_{i}
$$

by (7.4.3) and by the definitions of $L_{\imath}^{\prime \prime}$ and $\dot{\phi}_{i}$. Let $\delta_{i}^{\prime \prime \prime}$ be a positive small number such that $f^{*}$ is $P L$ on $f^{*-1}\left(\left[s_{1}, s_{1}+\delta_{i}^{\prime \prime \prime}\right] \cup\left[s_{2}-\delta_{i}^{\prime \prime \prime}, s_{2}\right]\right) \cap\left(M_{i}^{*} \cup M_{i-1}^{*} \cup M_{i+1}^{*}\right)$ and that

$$
\left(\left(s_{1}, s_{1}+\delta_{2}+\delta_{\imath}^{\prime \prime \prime}\right] \cup\left[s_{2}-\delta_{i}-\delta_{\imath}^{\prime \prime \prime}, s_{2}\right)\right) \cap\left(S_{i+1} \cup S_{i-1}\right)=\varnothing .
$$

We want to find a homeomorphism $\tau_{3 C}$ of $\bar{Q}$ such that $\tau_{3 C}=$ ident on $(\bar{Q}-Q) \cup$ $\Theta^{*}\left(\bar{C} \times\left[\delta_{i}^{\prime \prime \prime}, s_{2}-s_{1}-2 \delta_{1}-\delta_{i}^{\prime \prime \prime}\right]\right) \cup\left(\right.$ a neighborhood of $X^{*} \cap \bar{Q}$ in $\left.\bar{Q}\right)$ and that $f^{*}{ }^{\circ} \tau_{3 C}$ is $P L$ on $Q$. In (7.8) we will shorten $\delta_{i}^{\prime \prime \prime}$ moreover. Put $Q_{1}=f^{*-1}\left(\left[s_{1}+\delta_{i}^{\prime \prime \prime}, s_{1}+\delta_{i}\right.\right.$ $\left.\left.+\delta_{\imath}^{\prime \prime \prime}\right]\right) \cap Q$. Let $\chi: C \times[0,1] \rightarrow Q_{1}$ be a homeomorphism defined by $\chi(x, s)=\Theta^{*}(x$, $\left.\delta_{l}^{\prime \prime \prime}-s \delta_{i}\right)$. Then we have

$$
\begin{aligned}
& \chi(C \times 1)=f^{*-1}\left(s_{1}+\delta_{i}^{\prime \prime \prime}\right) \cap Q_{1}, \\
& \chi(C \times 0)=f^{*-1}\left(s_{1}+\delta_{i}+\delta_{2}^{\prime \prime \prime}\right) \cap Q_{1}, \\
& \chi\left(C \times\left[0, \delta_{i}^{\prime \prime \prime} / \delta_{i}\right]\right)=\mid K_{1} ! \cap Q_{1},
\end{aligned}
$$

$\left.f^{*} \circ\right|_{x \times[0,1]}$ is linear, and the restriction of $\chi$ to $C \times\left[0, \delta_{2}^{\prime \prime \prime} / \delta_{i}\right]$ is a $P L$ homeomorphism to $\left|K_{1}\right| \cap Q_{1}$ by $(7.4 .3,10)$. Let $\tilde{\chi}: \bar{C} \times[0,1] \rightarrow \bar{Q}_{1}$ be the continuous extension of $\chi$, whose existence is trivial.

Recall the following well-known facts. Let $A \subset B$ be compact polyhedrons, and let $\zeta$ be a $P L$ function on $B$ such that $\zeta^{-1}(0)=A$. Then there exists a positive number $c$ such that for any $0<c^{\prime}<c, \zeta^{-1}\left(\left[-c^{\prime}, c^{\prime}\right]\right)$ is a regular neighborhood of $A$ in $B$. See [13] for the definition of regular neighborhood from which the above fact follows easily. If $B$, moreover, is a $P L$ manifold (with boundary), then any regular neighborhood is a $P L$ manifold with boundary (Corollary $3.30,[13])$.

Consider a compact polyhedron pair of a small closed neighborhood of $\bar{Q}$ in $M^{*}$ which is a $P L$ manifold with boundary and the intersection of the neighborhood and $f^{*-1}\left(s_{1}\right) \cup f^{*-1}\left(s_{1}+\delta_{i}\right)$. Then, by the above facts, the intersection of the neighborhood and $f^{*-1}\left(\left(s_{1}, s_{1}+\delta_{i}^{\prime \prime \prime}\right] \cup\left(s_{1}+\delta_{\imath}, s_{1}+\delta_{2}+\delta_{\imath}^{\prime \prime \prime}\right]\right)$ is a $P L$ manifold with boundary for small $\delta_{i}^{\prime \prime \prime}$. Therefore, since

$$
Q_{1}=Q-f^{*-1}\left(\left(s_{1}, s_{1}+\delta_{i}^{\prime \prime \prime}\right) \cup\left(s_{1}+\delta_{\iota}+\delta_{2}^{\prime \prime \prime}, s_{2}\right)\right),
$$

we assume that $Q_{1}$ is a $P L$ manifold with boundary. Hence we can apply Lemma 7.1 to $\chi: C \times[0,1] \rightarrow Q_{1}$.

Let, accordingly, $\chi_{t}: C \times[0,1] \rightarrow Q_{1}, 0 \leqq t \leqq 1$, be an isotopy of $\chi$ such that

(7.7.1) $\chi_{1}$ is a $P L$ homeomorphism,

(7.7.2) $\chi_{t}=\chi, 0 \leqq t \leqq 1$, on $C \times 0$ and that

(7.7.3) for all $t, \chi_{t}$ are close approximations of $\chi$ in the $C^{0}$ fine topology. Hence the isotopy can be extended to an isotopy $\tilde{\chi}_{t}: \bar{C} \times[0,1] \rightarrow \bar{Q}_{1}, 0 \leqq t \leqq 1$, by putting 


$$
\tilde{\chi}_{t}(x, s)=\tilde{\chi}(x, s) \quad \text { for } \quad x \in \bar{C}-C, 0 \leqq s \leqq 1 .
$$

Now we define $\tau_{3 C}$ on $Q_{2}=f^{*-1}\left(\left[s_{1}+\delta_{2}^{\prime \prime \prime} / 2, s_{1}+\delta_{\imath}+\delta_{2}^{\prime \prime \prime}\right]\right) \cap Q$. Put

$$
\tau_{: C}(x)=\left\{\begin{array}{l}
\chi_{\circ} \chi_{1}^{-1}(x) \quad \text { on } Q_{1}-f^{*-1}\left(s_{1}+\delta_{\imath}^{\prime \prime \prime}\right), \\
\Theta^{*}\left(\chi_{\circ} \chi_{t}^{-1} \circ \Theta^{*}\left(x, s_{1}+\delta_{\imath}^{\prime \prime \prime}-f^{*}(x)\right), f^{*}(x)-s_{1}-\delta_{\imath}^{\prime \prime \prime}\right) \text { on } Q_{2}-\operatorname{Int} Q_{1}
\end{array}\right.
$$

where

$$
t=2\left(f^{*}(x)-s_{1}-\delta_{i}^{\prime \prime \prime} / 2\right) / \delta_{\imath}^{\prime \prime \prime} .
$$

We remark that $Q_{2}-$ Int $Q_{1} \ni x \rightarrow \Theta^{*}\left(x, s_{1}+\delta_{i}^{\prime \prime \prime}-f^{*}(x)\right) \in M^{*}$ is a projection onto $\chi(C \times 1)=f^{*-1}\left(s_{1}+\delta_{i}^{\prime \prime \prime}\right) \cap Q$, that $\left.\chi_{t}\right|_{C \wedge 1}, 0 \leqq t \leqq 1$, are homeomorphisms onto $\chi(C \times 1)$ and that $f^{*}{ }_{\circ} \tau_{3 C}(x)=f^{*}(x)$ for $x \in Q_{2}-$ Int $Q_{1}$. Hence $\left.\tau_{3 C}\right|_{Q_{2}-\text { Int } Q_{1}}$ is a homeomorphism of $Q_{2}$-Int $Q_{1}$, coincides with $\chi_{\circ} \chi_{1}^{-1}$ on $\chi(C \times 1)$, equals the identity on $Q_{2}-$ Int $Q_{2}-Q_{1}=f^{*-1}\left(s_{1}+\delta_{i}^{\prime \prime \prime} / 2\right) \Gamma Q$ and is a close approximation of the identity in the $C^{0}$ fine topology by (7.7.3). Consequently $\tau_{3 C}$ is a homeomorphism of $Q_{2}$ close to the identity. It trivially follows also that $f^{*} \tau_{3 C}$ is $P L$ on $Q_{2}-\operatorname{Int} Q_{1}$. Look into $\tau_{3 C}$ on $Q_{1}$ in detail. $\left.f^{*} \tau_{3 C}\right|_{Q_{1}}$ is $P L$ since so are $f^{*} \chi^{\circ}$ and $\chi_{1}^{-1}$. From (7.4.10) $)^{\prime}$ and from the equality $\Theta^{*}\left(\left(\Theta *\left(C \times \delta_{2}^{\prime \prime \prime}\right)\right) \times\left[-\delta_{i}, 0\right]\right)=Q_{1}$ it follows that $\left.\chi^{-1} \circ \Theta^{*}\right|_{\Theta *\left\{C \times \delta_{2}^{\prime \prime \prime}\right) \times\left[-\tilde{o}_{i}, 0\right]}$ is a $P L$ homeomorphism onto $C \times[0,1]$. Hence $\tau_{3 C^{\circ}}^{-1} \Theta^{*}$ is $P L$ on $\Theta^{*}\left(C \times \delta_{i}^{\prime \prime}\right) \times\left[-\delta_{\iota}, 0\right]$. We remark that $\tau_{3 C}^{-1} \circ \Theta^{*}=\tau_{3 C}^{-1} \circ \Theta^{*} \circ\left(\tau_{3 C}\right.$, ident $)$ or $\Theta^{*}\left(C \times \delta_{i}^{\prime \prime \prime}\right) \times\left[-\delta_{i}, 0\right]$.

Define $\tau_{3 C}$ also on $f^{*-1}\left(\left[s_{2}-\delta_{2}-\delta_{i}^{\prime \prime \prime}, s_{2}-\delta_{i}^{\prime \prime \prime} / 2\right]\right) \cap Q$ in the same way as above, and extend it to $\bar{Q}$ by putting $\tau_{3 C}=$ ident on $\left(f^{*-1}\left(\left[s_{1}, s_{1}+\delta_{l}^{\prime \prime \prime} / 2\right) \cup\left(s_{1}+\delta_{2}+\delta_{2}^{\prime \prime \prime}, s_{2}\right.\right.\right.$ $\left.\left.\left.-\delta_{\imath}-\delta_{i}^{\prime \prime \prime}\right) \cup\left(s_{2}-\delta_{i}^{\prime \prime \prime} / 2, s_{2}\right]\right) \cap Q\right) \cup(\bar{Q}-Q)$. Then $f^{*} \circ \tau_{3 C}$ is $P L$ on $Q, \tau_{3 C}^{-1} \circ \Theta^{*} \circ\left(\tau_{3 C}\right.$, ident $)$ is $P L$ on $\Theta^{*}\left(C \times \delta_{2}^{\prime \prime \prime}\right) \times\left[-\delta_{i}, s_{2}-s_{1}-\delta_{2}-2 \delta_{2}^{\prime \prime \prime}\right]$ by $(7.4 .10)^{\prime}$, and $\tau_{3 C}$ is a close approximation of the identity in the $C^{0}$ (fine) topology. We remark that if we put $\tau_{3 C}=$ ident outside $\bar{Q}$ then we can extend $\tau_{3 C}$ globally to $M^{*}$.

Repeat this argument for each connected component of $L^{\prime \prime *}-\partial L^{\prime \prime *}$. Then we have a homeomorphism $\tau_{3}$ of $M^{*}$ close to the identity and equal to it outside $\Theta^{*}\left(\left(L^{\prime \prime *}-\partial L^{\prime \prime *}\right) \times \boldsymbol{R} \cap D^{*}\right)$ such that $f^{*} \circ \tau_{3}$ is $P L$ on $\Theta^{*}\left(\left(L^{\prime \prime *}-\partial L^{\prime \prime *}\right) \times \boldsymbol{R} \cap D^{*}\right) \cup$ (a neighborhood of $\left.X^{*}\right)$. Replace $f^{*}, \Theta^{*}, L^{\prime \prime *},\left|K_{1}\right|$, etc. by $f^{*} \circ \tau_{3}, \tau_{3}^{-1} \circ \Theta^{*} \circ\left(\tau_{3}\right.$, ident), $\bigcup_{C} \Theta^{*}\left(\bar{C} \times \delta_{\imath}^{\prime \prime \prime}\right), \bigcup_{C} \Theta^{*}\left(\bar{C} \times\left[\delta_{\imath}^{\prime \prime \prime}, s_{2}-s_{1}-2 \delta_{\imath}-\delta_{\imath}^{\prime \prime \prime}\right]\right)$, etc. respectively, and use the former notations for them. Then we can assume

$$
f^{*} \text { is } P L \text { on } \Theta^{*}\left(\left(L^{\prime \prime *}-\partial L^{\prime \prime *}\right) \times \boldsymbol{R} \cap D^{*}\right) \text {, and }
$$

(7.7.5) $\Theta^{*}$ is $P L$ on $C \times\left[\delta_{l}^{\prime \prime \prime}-\delta_{i}, s_{2}-s_{1}-\delta_{\imath}-\delta_{\imath}^{\prime \prime \prime}\right]$ for any connected component $C$ of $L^{\prime \prime *}-\partial L^{\prime \prime *}$ where $\delta_{i}, \delta_{\imath}^{\prime \prime \prime}, s_{1}$ and $s_{2}$ are given as before.

Clearly (7.7.5) is equivalent to

(7.7.6) $\Theta^{*}$ is $P L$ on $\Theta^{*}(C \times c) \times\left[\delta_{\imath}^{\prime \prime \prime}-\delta_{\imath}-c, s_{2}-s_{1}-\delta_{\imath}-\delta_{\imath}^{\prime \prime \prime}-c\right]$ for any $c \in\left[\delta_{\imath}^{\prime \prime \prime}-\right.$ $\left.\hat{o}_{1}, s_{2}-s_{1}-\delta_{i}-\delta_{i}^{\prime \prime \prime}\right]$.

(7.8) Finally we piecewise linearize $f^{*}$ globally. For it we only need to do so on $M^{*}-X^{*}-\Theta^{*}\left(L^{\prime *} \times \boldsymbol{R} \cap D^{*}\right)$ because of (7.6.1) and (7.7.4). Let $U$ be a connected component of $M^{*}-X^{*}-\Theta^{*}\left(L^{\prime *} \times \boldsymbol{R} \cap D^{*}\right)$. Then it is contained in 
some $M_{j}^{*}, j$ : even, because for each odd $i, M_{i}^{*}-X^{*}$ is contained in $\Theta^{*}\left(\left(L^{\prime *}-\right.\right.$ $\left.\left.\partial L^{\prime *}\right) \times \boldsymbol{R} \cap D^{*}\right)$ by (7.4.8). Put $f^{*}(U)=\left(s_{1}, s_{2}\right)$. Let $x \in U$. Then

$$
\Theta *\left(x \times \boldsymbol{R} \cap D^{*}\right) \subset M_{j}^{*}
$$

by (7.4.8), and $s_{1}, s_{2} \in S_{\jmath}$. Hence, by (7.4.3),

$$
x \times \boldsymbol{R} \cap D^{*} \supset x \times\left(s_{1}-f^{*}(x), s_{2}-f^{*}(x)\right),
$$

which is equivalent to

$$
\begin{aligned}
\Theta^{*}\left(x \times \boldsymbol{R} \cap D^{*}\right) \cap U & \left.=\text { (some connected component of } \Theta^{*}\left(x \times \boldsymbol{R} \cap D^{*}\right)-X^{*}\right) \\
& =\Theta *\left(x \times\left(s_{1}-f^{*}(x), s_{2}-f^{*}(x)\right)\right) .
\end{aligned}
$$

Hence we obtain the following. Let $s \in\left(s_{1}, s_{2}\right)$ and put $C=f^{*-1}(s) \cap U$. Then $C$ is a topological manifold, $C \times\left(s_{1}-s, s_{2}-s\right) \subset D^{*}$, and $\left.\Theta^{*}\right|_{C}\left(s_{1}-s, s_{2}-s\right): C \times\left(s_{1}-\right.$ $\left.s, s_{2}-s\right) \rightarrow U$ is a homeomorphism. Let $C_{1}$ be a closed subset of $C$ such that $C_{1} \cup(\bar{C}-C)$ is a neighborhood of $\bar{C}-C$ in $\bar{C}$ and that

$$
C_{1} \subset \Theta^{*}\left(\left(L^{\prime \prime *}-\partial L^{\prime \prime *}\right) \times \boldsymbol{R} \cap D^{*}\right) .
$$

Then, by (7.6.1) and (7.7.4) we have $\lambda_{j}>0$ such that

(7.8.2) $\left.f^{*}\right|_{U}$ is $P L$ on a neighborhood of $\Theta *\left(C_{1} \times\left(s_{1}-s, s_{2}-s\right)\right) \cup\left(U-f^{*}\left(\left(s_{1}+\lambda_{y}\right.\right.\right.$, $\left.\left.s_{2}-\lambda_{\jmath}\right)\right)$.

Put $s=s_{2}-\lambda_{\jmath}$, and shorten $\delta_{j-1}^{\prime \prime \prime}$ and $\delta_{j+1}^{\prime \prime \prime}$ defined in (7.7) so that $\lambda_{\jmath}>\delta_{\jmath-1}^{\prime \prime \prime}$, $\delta_{j+1}^{\prime \prime \prime}$. Then $C$ is a $P L$ manifold, and it follows from (7.7.6), (7.8.1) and from the inclusion $S_{j} \supset S_{j-1} \cup S_{j+1}$ that

(7.8.3) $\Theta^{*}$ is $P L$ on a neighborhood of $C_{1} \times\left[-s_{2}+s_{1}+2 \lambda_{3}, 0\right]$ in $C \times\left[-s_{2}+s_{1} \div\right.$ $\left.2 \lambda_{j}, 0\right]$.

Put

$$
U_{1}=\Theta^{*}\left(C \times\left[-s_{2}+s_{1}+2 \lambda_{\jmath}, 0\right]\right)=U \cap f^{*-1}\left(\left[s_{1}+\lambda_{\jmath}, s_{2}-\lambda_{\jmath}\right]\right) .
$$

Consider a compact polyhedron pair of a small closed neighborhood $V$ of $\bar{U} \cap f^{*-1}\left(\left\{s_{1}, s_{2}\right\}\right)$ in $M^{*}$ which is a $P L$ manifold with boundary and $V \cap f^{*-1}$ $\left(\left\{s_{1}, s_{2}\right\}\right)$. Then, by the facts about regular neighborhood stated in (7.7) and by (7.6.1), $V-f^{*-1}\left(\left(s_{1}+\lambda_{j}, s_{2}-\lambda_{j}\right)\right)$ is a $P L$ manifold with boundary for small $\lambda_{j}$. Moreover we have a $P L$ homeomorphism $\mu:\left(V \cap f^{*-1}\left(s_{2}-\lambda_{j}\right)\right) \times[0,1) \rightarrow V \cap$ $f^{*-1}\left(\left[s_{2}-\lambda_{j}, s_{2}\right)\right)$ for small $\lambda_{j}$ such that

$$
\begin{aligned}
& \mu(x, 0)=x \text { and } \quad f^{*} \mu_{\varphi} \mu(x, t)=s_{2}-\lambda_{j}+t \lambda_{j} \\
& \text { for } \quad x \in V \cap f^{*-1}\left(s_{2}-\lambda_{j}\right), t \in[0,1),
\end{aligned}
$$

which follows easily from the proof of the fact that $V \cap f^{*-1}\left(\left[s_{2}-\lambda_{j}, s_{2}+\lambda_{j}\right]\right)$ is a regular neighborhood of $V \cap f^{*-1}\left(s_{2}\right)$ in $V$. These imply that 
(7.8.4) we can assume $U_{1}$ and $C$ are $P L$ manifolds with boundary and without boundary respectively

and that we can modify $\left.\Theta^{*}\right|_{C \times\left[0, \lambda_{j}\right)}$ fixing it on $C \times 0$ so that $\Theta^{*}$ is $P L$ there. If we replace $C$ by $\Theta^{*}\left(C \times \lambda_{\jmath}^{\prime}\right)$ for sufficiently small $\lambda_{\jmath}^{\prime}>0$, the last statement means that we can assume

(7.8.5) $\Theta^{*}$ is $P L$ on a neighborhood of $C \times 0$ in $C \times\left[-s_{2}+s_{1}+2 \lambda_{3}, 0\right]$.

According to $(7.8 .3,4,5)$ we can apply Lemma 7.1 . To be exact, let a homeomorphism $\omega: C \times[0,1] \rightarrow U_{1}$ be defined by

$$
\omega(x, u)=\Theta^{*}\left(x, u\left(-s_{2}+s_{1}+2 \lambda_{\jmath}\right)\right) .
$$

Then $\omega$ is $P L$ on a neighborhood of $C \times 0 \cup C_{1} \times[0,1]$. Hence, by Lemma 7.1 we have an isotopy $\omega_{t}: C \times[0,1] \rightarrow U_{1}, 0 \leqq t \leqq 1$, of $\omega$ such that

(7.8.6) $\omega_{1}$ is $P L$,

(7.8.7) $\omega_{t}=\omega, 0 \leqq t \leqq 1$, on a neighborhood of $C \times 0 \cup C_{1} \times[0,1]$ and that

(7.8.8) for all $t, \omega_{t}$ are close approximations of $\omega$.

We define a homeomorphism $\tau_{1 C}$ of $U_{2}=f^{*-1}\left(\left[s_{1}+\lambda_{j} / 2, s_{2}-\lambda_{j}\right] \cap U\right.$ in the same way as $\tau_{3 c}$, namely, put

where

$$
\tau_{\sharp C}(x)=\left\{\begin{array}{rr}
\omega^{\circ} \omega_{1}^{-1}(x) \quad \text { for } \quad x \in U_{1}-f^{*-1}\left(s_{1}+\lambda_{\jmath}\right) \\
\Theta^{*}\left(\omega^{\circ} \omega_{t}^{-1} \circ \Theta^{*}\left(x, s_{1}+\lambda_{3}-f^{*}(x)\right), f^{*}(x)-s_{1}-\lambda_{\jmath}\right) \\
\text { for } x \in U_{2}-\operatorname{Int} U_{1}
\end{array}\right.
$$

$$
t=2\left(f^{*}(x)-s_{1}-\lambda_{\jmath} / 2\right) / \lambda_{\jmath} .
$$

Then, by the same reason as (7.7),

(7.8.9) $\tau_{4 C}$ is well-defined, the identity on $U_{2}-\operatorname{Int} U_{2}$, and close to the identity on $U_{2}$,

(7.8.10) $f^{*}{ }^{\circ} \tau_{4 C}=f^{*}$ on $U_{2}-U_{1}$, and $f^{*}{ }^{*} \tau_{\ddagger C}$ is $P L$ on $U_{1}$. Hence

(7.8.11) $f^{*} \circ \tau_{4 C}$ is $P L$ on $U_{2}$.

Moreover it follows from (7.8.7) that

(7.8.12) $\tau_{4 C}$ is the identity on $\Theta^{*}\left(C_{1} \times\left[-s_{2}+s_{1}+3 \lambda_{j} / 2,0\right]\right)$.

Extend $\tau_{+C}$ to $U$ by putting $\tau_{1 C}=$ ident on $U-U_{2}$. Then, by $(7.8 .2,9,11,12)$

(7.8.13) $\tau_{4 C}$ is a homeomorphism of $U$, the identity outside a compact subset of $U$ and close to the identity, and $f^{*}{ }_{\circ} \tau_{\sharp C}$ is $P L$ on $U$.

Repeating this argument for each connected component of $M^{*}-X^{*}-\Theta^{*}\left(L^{\prime *}\right.$ $\left.\times \boldsymbol{R} \cap D^{*}\right)$, we obtain a homeomorphism $\tau_{4}$ of $M^{*}$ close to the identity and equal to it on a neighborhood of $X^{*} \cup \Theta^{*}\left(L^{*} \times \boldsymbol{R} \cap D^{*}\right)$ such that $f^{*} \circ \tau_{4}$ is $P L$ on $M^{*}$ 
$-X^{*}-\Theta^{*}\left(L^{* *} \times \boldsymbol{R} \cap D^{*}\right)$ (7.8.13). Since $f^{*}$ is $P L$ on a neighborhood of $X^{* * 1} J^{\prime}$ $\Theta^{*}\left(L^{* *} \times \boldsymbol{R} \cap D^{*}\right)$ (7.6.1), (7.7.4), it follows that $f^{*} \circ \tau_{4}$ is globally $P L$. Hence we complete the proof of Theorem I.

\section{$\S 8$. Proofs of the Other Results}

As Theorem II is a special case of Theorem II' (see Proposition 6.11), we do not need to prove Theorem II.

(8.1) Proof of Theorem $I^{\prime}$. Let $M$ be closed in $\boldsymbol{R}^{n}$, and let $\tilde{M} \subset \boldsymbol{R}^{n} \times \boldsymbol{R}$ be the graph of $f$. Then $\tilde{M}$ is a closed subanalytic set in $\boldsymbol{R}^{n} \times \boldsymbol{R}$. Let $\tilde{f}: \tilde{M} \rightarrow \boldsymbol{R}$ be the restriction of the projection $\boldsymbol{R}^{n} \times \boldsymbol{R} \rightarrow \boldsymbol{R}$. We remark thar $\tilde{f}$ is proper, since $f$ is so. Recall the proof of Proposition 5.1. (5.3) tells us the following. There exist a Whitney subanalytic stratification $\left\{\tilde{M}_{i}\right\}_{i=1, \ldots}$ of $\tilde{M}$, compact analytic manifolds $N_{i}, i=1,2, \cdots$, subanalytic open subsets $N_{i}^{\prime} \subset N_{i}, i=1, \cdots$, and analytic maps $\phi_{i}: N_{i} \rightarrow \tilde{M}, i=1, \cdots$, such that

(8.1.1) for each $i,\left.\phi_{i}\right|_{\nu_{\imath}^{\prime}}$ is a diffeomorphism onto $\tilde{M}_{\imath}$.

Put for each $i$

$$
\tilde{f}_{\imath}=\left.\tilde{f}\right|_{\tilde{I}_{\imath}}, S_{\imath}=\left\{x \in \tilde{M}_{\imath} \mid d \tilde{f}_{i x}=0\right\} \text {. }
$$

Then $S_{i}$ coincides with $S\left(\tilde{M}_{i}, n+1\right)$ for $f_{1}=f_{2}=\tilde{f}$ in (5.3). Hence, by (5.3.6), $S_{\iota}$ is closed in $\tilde{M}_{i}$ and subanalytic. Here the condition (8.1.1) is important. It follows from (2.5) that $\tilde{f}_{\imath}\left(S_{i}\right)$ is a subanalytic set in $\boldsymbol{R}$, which clearly is bcunded and of dimension 0 , since $\tilde{M}_{i}$ is bounded. Let $R$ denote the union of all $\tilde{f}_{i}\left(S_{l}\right)$, and put

$$
\widetilde{S}=\tilde{f}^{-1}(R), \quad S=f^{-1}(R) .
$$

Then the properness of $\tilde{f}$ assures that $R$ is a discrete set, hence $S$ and $\tilde{S}$ are closed subanalytic sets in $\boldsymbol{R}^{n}, \boldsymbol{R}^{n+1}$ respectively by (2.5). Adding $\boldsymbol{Z}$ to $R$ if necessary, we assume $R$ to be unbounded from above and below..

Consider the vector field $\xi=\left\{\xi_{i}=d \tilde{f}_{i}\right\}$ on $\left\{\tilde{M}_{i}-\tilde{S}\right\}$ (see Definition 4.10). Clearly $\left\{\tilde{M}_{i}-\tilde{S}\right\}$ is a Whitney stratification of a locally closed subsets $\tilde{M}-\tilde{S}$ of $\boldsymbol{R}^{n+1}$, and we have $\xi_{\imath}\left(\tilde{f} i_{\tilde{H}_{i}-\tilde{S}}\right)>0, i=1,2, \cdots$. Put $f_{1}=f_{2}=\tilde{f}, V_{0}=V_{0}^{\prime}=\boldsymbol{R}^{n+1}, V_{\jmath}=$ $V_{j}^{\prime}=\varnothing$ for $1 \leqq j \leqq n$. Then $\xi, \tilde{M}-\tilde{S}, f_{1}, f_{2}, V_{j}$ and $V_{\jmath}^{\prime}, 0 \leqq j \leqq n$, satisfy the conditions in Lemma 4.14. Hence we have a locally integrable vector field $\xi^{\prime}=\left\{\xi_{\imath}^{\prime}\right\}$ on $\left\{\tilde{M}_{i}-\tilde{S}\right\}$ such that $\xi_{i}^{\prime}\left(\left.\tilde{f}\right|_{\tilde{H}_{i}-\tilde{S}}\right)>0, i=1,2, \cdots$. After multiplying $\xi_{i}^{\prime}$ by $1 / \xi_{i}^{\prime} \tilde{f}$, we assume moreover

$$
\xi_{\imath}^{\prime}\left(\left.\tilde{f}\right|_{\tilde{M}_{i}-\tilde{S}}\right)=1, i=1,2, \cdots .
$$

Let $\widetilde{\Theta}: \tilde{D} \rightarrow \tilde{M}-\tilde{S}$ be the flow of $\xi^{\prime}$ (see Definition 4.12). Then, since $\xi^{\prime}$ is locally integrable, $\tilde{D}$ is open in $(\tilde{M}-\tilde{S}) \times \boldsymbol{R}$, and $\Theta$ is continuous. (8.1.2) clearly implies 


$$
\tilde{f}(\tilde{\Theta}(x, t))=\tilde{f}(x)+t \quad \text { for } \quad(x, t) \in \tilde{D}
$$

Let $p: \boldsymbol{R}^{n} \times \boldsymbol{R} \rightarrow \boldsymbol{R}^{n}$ be the projection. Put

$$
\begin{aligned}
& D=\{(p(x), t) \mid(x, t) \in \tilde{D}\}, \\
& \Theta(x, t)=p \circ \widetilde{\Theta}\left(x, \int(x), t\right) \quad \text { for } \quad(x, t) \in D .
\end{aligned}
$$

Then $D$ is open in $(M-S) \times \boldsymbol{R}$, and $\Theta: D \rightarrow M-S$ is a continuous flow such that by (8.1.3)

$$
f(\Theta(x, t))=f(x)+t \quad \text { for } \quad(x, t) \in D .
$$

Let $U$ be a connected component of $M-S$. Put $f(U)=\left(s_{1}, s_{2}\right)$. Then $s_{1}, s_{2}$ $\in R$. Let $s \in\left(s_{1}, s_{2}\right)$, and put $C=f^{-1}(s) \cap U$. Then, since $f$ is proper, $U$ is bounded, $C$ is compact, hence we have $C \times \boldsymbol{R} \cap D=C \times\left(s_{1}-s, s_{2}-s\right)$, and $\left.\Theta\right|_{C<\left(s_{1}-s, s_{2}-s\right)}: C \times\left(s_{1}-s, s_{2}-s\right) \rightarrow U$ is a homeomorphism.

In the same way as $(7.5,6)$ we can reduce the problem to the case in which $S$ is a subpolyhedron of $M$ and $f$ is $P L$ on a neighborhood of $S$. Here the importance is that $S$ is closed in $M$ and that for any $x \in S, f^{-1} f(x) \equiv S$ as germs at $x$. We do not repeat the proof of reduction. For each above $U$, there exists $\lambda>0$ such that $f$ is $P L$ on a neighborhood of $\bar{U}-f^{-1}\left(\left(s_{1}+\lambda, s_{2}-\lambda\right)\right)$, since $\bar{U}$ is compact. Choose $\lambda$ so small that $U \cap f^{-1}\left(\left[s_{1}+\lambda, s_{2}-\lambda\right]\right)$ is a $P L$ manifold (see (7.7)), and put $s=s_{2}-\lambda$. Then $U \cap f^{-1}\left(\left[s_{1}+\lambda, s_{2}-\lambda\right]\right)$ and $C$ are compact $P L$ manifolds with boundary and without boundary respectively. It is easier than (7.7) to find a homeomorphism $\tau_{U}$ of $U \cap f^{-1}\left(\left[s_{1}+\lambda / 2, s_{2}-\lambda\right]\right)$ such that $f \circ \tau_{U}$ is $P L$ there and that $\tau_{U}=$ ident on $U \cap f^{-1}\left(\left\{s_{1}+\lambda / 2, s_{2}-\lambda\right\}\right)$. We omit the details. Extend $\tau_{U}$ to $M$ by putting $\tau_{U}=$ ident outside $U \cap f^{-1}\left(\left[s_{1}+\lambda / 2, s_{2}-\lambda\right]\right)$. Then $f \circ \tau_{U}$ is a subanalytic function on $M$ and $P L$ on a neighborhood of $\bar{U}$. Repeating this argument for each component of $M-S$, we obtain a homeomorphism $\tau$ of $M$ such that $f \circ \tau$ is $P L$ on $M$. Here $\tau$ can be chosen to be arbitrarily close to the identity by the same reason as the proof of Theorem I. Hence Theorem $\mathrm{I}^{\prime}$ is proved.

Remark 8.2. The reason why I assumed the properness of $f$ in Theorem $\mathrm{I}^{\prime}$ is that I could not modify (7.3) for the case of subanalytic $f$, nor I could proceed with the proof without the modification in the non-proper case.

(8.3) Proof of Theorem $I I^{\prime}$. When we proceed with the proof in the same way as (8.1), there is no problem except in the last step, so we assume the following.

(8.3.1) $R \subset \boldsymbol{R}$ is a discrete set unbounded from above and below.

(8.3.2) $S=f^{-1}(R)$ is a subpolyhedron of $M I$ such that $f$ is $P L$ on a neighborhood of $S$.

(8.3.3) $\Theta: D \rightarrow M-S, D \subset(M-S) \times \boldsymbol{R}$, is a continuous flow such that 


$$
f \circ \Theta(x, t)=f(x)+t \quad \text { for } \quad(x, t) \in D
$$

and that for any connected component $U$ of $M-S$ with $f(U)=\left(s_{1}, s_{2}\right)$ and for any $s \in\left(s_{1}, s_{2}\right)$ we have $C \times \boldsymbol{R} \cap D=C \times\left(s_{1}-s, s_{2}-s\right)$, and $\left.\Theta\right|_{C 、\left(s_{1}-s, s_{2}-\dot{s}\right)}: C \times$ $\left(s_{1}-s, s_{2}-s\right) \rightarrow U$ is a homeomorphism where $C=U \cap f^{-1}(s)$.

(8.3.4) $\lambda$ is a small positive number depending on $U$ such that $f$ is $P L$ on a neighborhood of $\bar{U}-f^{-1}\left(\left(s_{1}+\lambda, s_{2}-\lambda\right)\right)$ and that $U \cap f^{-1}\left(\left[s_{1}+\lambda, s_{2}-\lambda\right]\right)$ is a $P L$ manifold.

For the above $U$, let $s=s_{1}+\lambda, C^{\prime}=U \cap f^{-1}\left(s_{2}-\lambda\right)$. Then, by (8.3.4), $M-U \cap$ $f^{-1}\left(\left(s_{1}+\lambda, s_{2}-\lambda\right)\right)$ is a $P L$ manifold with boundary $C \cup C^{\prime}$. As we can not apply Lemma 7.1 in the same way as (7.7), we need the following result of Moise (see p. 15, [6]). Let $\phi: C \rightarrow C^{\prime}$ be a homeomorphism. Then, there exists an isotopy $\phi_{t}, 0 \leqq t \leqq 1$, of $\phi$ such that $\phi_{1}$ is $P L$. Consider the case in which $\phi(x)$ $=\Theta\left(x, s_{2}-s_{1}-2 \lambda\right), x \in C$. By (8.3.3) $\phi$ is a homeomorphism onto $C^{\prime}$. Let $\phi_{t}$ be the above isotopy of $\phi$.

Let $M_{U}$ be the quotient space of $M-U \cap f^{-1}\left(\left(s_{1}+\lambda, s_{2}-\lambda\right)\right)$ by the identification of $x \in C$ with $\phi_{1}(x) \in C^{\prime}$. Then, since $\phi_{1}$ is a $P L$ homeomorphism, $M_{U}$ has naturally a $P L$ manifold structure. We want to find a homeomorphism $\tau_{U}: M_{U}$ $\rightarrow M$ such that

$$
\tau_{U}=\text { ident on a neighborhood of } M-U \text { and that }
$$$$
\text { (8.3.6) } f \circ \tau_{U} \text { is } P L \text { on } \tau_{U}^{-1}(U) \text {. }
$$

If it is possible, then repeating the construction of $M_{U}$ and $\tau_{U}$ for each connected component $U$ of $M-S$, we obtain a $P L$ manifold $M^{\prime}$ and a homeomorphism $\tau$ : $M^{\prime} \rightarrow M$ such that $f \circ \tau$ is $P L$.

We define at first a map $\tilde{\tau}_{U}: M-U \cap f^{-1}\left(\left(s_{1}+\lambda, s_{2}-\lambda\right)\right) \rightarrow M$ by

$$
\tilde{\tau}_{U}(x)=\left\{\begin{array}{c}
x \quad \text { for } \quad x \in M-U \cap f^{-1}\left(\left[s_{1}+\lambda / 2, s_{2}-\lambda\right)\right) \\
\Theta\left(\psi_{t} \circ \Theta\left(x, s_{1}+\lambda-f(x)\right),-2\left(s_{2}-s_{1}-3 \lambda / 2\right)\left(s_{1}+\lambda-f(x)\right) / \lambda\right) \\
\text { for } x \in U \cap f^{-1}\left(\left[s_{1}+\lambda / 2, s_{1}+\lambda\right]\right)
\end{array}\right.
$$

where

$$
t=2\left(f(x)-s_{1}-\lambda / 2\right) / \lambda .
$$

Then it is well-defined and continuous because of

$$
\begin{aligned}
& \left(x, s_{1}+\lambda-f(x)\right) \in D \text { and } \Theta\left(x, s_{1}+\lambda-f(x)\right) \in C \\
& \text { for } x \in U \cap f^{-1}\left(\left[s_{1}+\lambda / 2, s_{1}+\lambda\right]\right), \\
& \tilde{\tau}_{U}(x)=x \quad \text { for } \quad x \in U \cap f^{-1}\left(s_{1}+\lambda / 2\right) .
\end{aligned}
$$

We also have, by (8.3.3), 


$$
f \circ \tilde{\tau}_{U^{\prime}}(x)=\left\{\begin{array}{c}
f(x) \text { on } \quad M-U \cap f^{-1}\left(\left[s_{1}+\lambda / 2, s_{2}-\lambda\right]\right) \\
s_{2}-\lambda-2\left(s_{2}-s_{1}-3 \lambda / 2\right)\left(s_{1}+\lambda-f(x)\right) / \lambda \text { on } \\
U \cap \mathcal{f}^{-1}\left(\left[s_{1}+\lambda / 2, s_{1}+\lambda\right]\right) .
\end{array}\right.
$$

Hence, by (8.3.4),

$$
f \circ \tilde{\tau}_{U} \quad \text { is } P L \text { on } \bar{U}-f^{-1}\left(\left(s_{1}+\lambda, s_{2}-\lambda\right)\right) .
$$

On the other hand

$$
\tilde{\tau}_{U}(x)=\Theta\left(\psi_{1} \circ \Theta(x, 0), 0\right)=\psi_{1}(x) \quad \text { for } \quad x \in U \cap f^{-1}\left(s_{1}+\lambda\right) .
$$

It is easy also to see that $\tilde{z}_{U}$ is surjective, and the restriction to $M-U \cap f^{-1}$ $\left(\left[s_{1}+\lambda, s_{2}-\lambda\right]\right)$ is one-to-one. Hence $\tilde{\tau}_{U}$ induces a homeomorphism $\tau_{U}: M_{U} \rightarrow M$. (8.3.5) is then clear by the definition of $\tilde{\tau}_{U}$, and (8.3.6) follows from (8.3.7). Hence Theorem II' is proved.

Remark 8.4. I do not know if $M^{\prime}$ in Theorems II, II $^{\prime}$ can be a manifold of $C^{\infty}$ triangulation of $M$.

(8.5) Proof of Corollary III. For each positive integer $m$, let $\Gamma_{m}$ denote the set of all simplicial complexes consisting of $m$ simplexes. We identify two complexes $K, L$ of $\Gamma_{m}$ if they are linearly isomorphic (see Definition 6.0). Then $\Gamma_{m}$ is a finite set.

For any complex $K \in \Gamma_{m}$, let $\Delta_{K}$ denote the set of all simplicial functions on $|K|$, namely functions on $|K|$ whose restriction on each simplex of $K$ is linear. We give a equivalence relation to $\Delta_{K}$ as follows. For $f, g \in \Delta_{K}$, we define $f \sim g$ if there is a homeomorphism $\tau$ of $\boldsymbol{R}$ such that $f=\tau \circ g$. Let $\tilde{J}_{K}$ denote the quotient set of $\Delta_{K}$ under the equivalence relation. Then it is easy to see that $\tilde{\Delta}_{K}$ is a finite set.

Let $\Delta_{m}$ and $\tilde{\Delta}_{m}$ denote the unions of $\Delta_{K}$ and $\tilde{\Delta}_{K}, K \in \Gamma_{m}$, respectively, and $p_{m}: \Delta_{m} \rightarrow \tilde{\Delta}_{m}$ be the projection. Put $\Delta=\bigcup_{m=1}^{\infty} \Delta_{m}, \tilde{\Delta}=\bigcup_{m=1}^{\infty} \tilde{\Delta}_{m}$, and let $p: \Delta \rightarrow \tilde{\Delta}$ be defined by $p=p_{m}$ on $\Delta_{m}$. Then $\tilde{\Delta}_{m}$ are finite sets, since $\tilde{\Delta}_{K}, \Gamma_{m}$ are finite sets, consequently $\tilde{\Delta}$ is countable.

Let $\Lambda$ denote the set of all subanalytic funcitons on $M$. For any $f \in \Lambda$, we have a simplical complex $K$ and a homeomorphism $\tau:|K| \rightarrow M$ such that $f \circ \tau \in$ $\Delta_{K}$ by Theorems I', II'. By putting $q(f)=p(f \circ \tau) \in \tilde{\Delta}$, we define a map $q: \Lambda \rightarrow \tilde{\Delta}$. If $f_{1}, f_{2} \in \Lambda$ are carried by $q$ to one element of $\tilde{\Delta}, f_{1}$ and $f_{2}$ clearly are topologically $R-L$ equivalent. Hence there are only countable topological $R-L$ equivalence classes of $\Lambda$. That proves Corollary III.

Remark 8.6. Corollary III does not hold true if $M$ is non-compact. In fact, the set of topological $R-L$ equivalence classes of all subanalytic (or analytic) functions on noncompact $M$ is a continuum. As the proof is easy, we omit it. 


\section{§9. Piecewise Linearization of Analytic Maps}

Let $f: M_{1}^{n} \rightarrow M_{2}^{m}$ be a real (or complex) analytic map between connected real (or complex) analytic manifolds respectively. We call $f$ piecewise linearizable if there are $C^{\infty}$ triangulations $\left(K_{1}, g_{1}\right),\left(K_{2}, g_{2}\right)$ of $M_{1}, M_{2}$ respectively and homeomorphisms $\tau_{1}:\left|K_{1}\right| \rightarrow M_{1}, \tau_{2}: M_{2} \rightarrow\left|K_{2}\right|$ such that $\tau_{2} \circ f_{\circ} \tau_{1}:\left|K_{1}\right| \rightarrow\left|K_{2}\right|$ is a $P L$ map (see the beginning of $\S 7$ ). Let $g: X_{1} \rightarrow X_{2}$ be a subanalytic map between subanalytic sets. For each $x \in X_{1}$, let $d_{g}(x)$ denote the dimension of the germ of $g^{-1} g(x)$ at $x$. Let $l$ be the maximal rank of $d f$ on $M_{1}$.

Proposition 9.1 (Real case). If $f$ is piecewise linearizable, there exists an analytic subset $X$ of $M_{1}$ of codimension at least two such that

$$
d_{f}(x)\left\{\begin{array}{lll}
=n-l & \text { for } & x \in M_{1}-X \\
\leqq n-l & \text { for } & x \in X .
\end{array}\right.
$$

Proof. At first we will prove $d_{f} \leqq n-l$ on $M_{1}$. Let $\left(K_{1}, g_{1}\right),\left(K_{2}, g_{2}\right)$ be $C^{\infty}$ triangulations of $M_{1}, M_{2}$ respectively, and $\tau_{1}:\left|K_{1}\right| \rightarrow M_{1}, \tau_{2}: M_{2} \rightarrow\left|K_{2}\right|$ be homeomorphisms such that $\tau_{2} \circ \circ \circ \tau_{1}$ is a $P L$ map. Imbed $K_{2}$ in some $\boldsymbol{R}^{m^{\prime}}$ so that $g=$ $\tau_{2} \circ f \circ \tau_{1}:\left|K_{1}\right| \rightarrow \boldsymbol{R}^{m^{\prime}}$ is a $P L$ map, and assume that for each simplex $\sigma$ of $K,\left.g\right|_{\sigma}$ is linear. Let $\sigma \in K, \Sigma$ be the affine space spanned by $\sigma$, and $G: \Sigma \rightarrow \boldsymbol{R}^{m^{\prime}}$ be the linear extension of $\left.g\right|_{\sigma}$. Then, for any $x \in \sigma, G^{-1} g(x)$ is an affine subspace of $\Sigma$, and we have $\left(\left.g\right|_{\sigma}\right)^{-1} g(x)=G^{-1} g(x) \cap \sigma$. Hence

(9.1.1) $d_{\left(\left.g\right|_{\sigma}\right)}$ is equal to $\operatorname{dim} \sigma-\operatorname{dim} g(\sigma)$ on $\sigma^{\circ}$ and hence constant there,

(9.1.2) for any $x \in \sigma, d_{\left(\left.g\right|_{\sigma}\right)}(x) \leqq d_{\left(\left.g\right|_{\sigma}\right)}(\stackrel{\circ}{\sigma})$.

Let $x \in\left|K_{1}\right|$. By the definition of $d_{g}, d_{g}(x)$ is the maximum of $d_{\left(\left.g\right|_{\sigma}\right)}(x)$ for $\sigma \in K$ with $x \in \sigma$. Here we can restrict $\sigma$ to $n$-simplexes, since $|K|$ is a $P L$ manifold. Hence, there is at least one $\sigma \in K$ of dimension $n$ such that $x \in \sigma$ and that $d_{g}(x)=d_{(g \mid \sigma)}(x)$. Hence, by (9.1.2) we obtain

$$
d_{g}(x) \leqq d_{(g \mid \sigma)}(\stackrel{\circ}{\sigma})=d_{g}(\stackrel{\circ}{\sigma}) .
$$

On the other hand, since the set of points $x \in M_{1}$ where $d f$ has rank $<l$ is an analytic subset of $M_{1}$ of codimension $\geqq 1$, we have $d_{g}=n-l$ on an open dense subset of $\left|K_{1}\right|$. Hence it follows from (9.1.3) that $d_{f} \leqq n-l$ on $M_{1}$.

By Proposition 9.3, [16], for any $x_{0} \in M_{1}, f$ takes the following form in some analytic local coordinate systems of $M_{1}, M_{2}$ around $x_{0}, f\left(x_{0}\right)$ respectively except on an analytic subset $X$ of $M_{1}$ of codimension $\geqq 2$.

$$
\begin{aligned}
& f(x)=\left(f_{1}(x), \cdots, f_{m}(x)\right), x=\left(x_{1}, \cdots, x_{n}\right), x_{0}=0, \\
& f_{1}(x)=x_{1}, \cdots, f_{p}(x)=x_{p}, \\
& f_{p+1}= \pm x_{p+1}^{s_{1}}
\end{aligned}
$$




$$
\begin{aligned}
& f_{p+2}=x_{p+2} x_{p+1}^{s_{2}}+f_{11} x_{p-1}^{s_{1}}, \\
& \quad \vdots \\
& f_{l}=x_{l} x_{p+1}^{s_{l-1}}+f_{l-p-1 l-p-1} x_{l-1} x_{p+1}^{s_{l-1} p-1}+\cdots+f_{l-\mu_{1-1}-\cdots,-} \\
& f_{l+1}=f_{l-p l-p} x_{l} x_{p+1}^{s_{l-1}}+\cdots+f_{l-p 1} x_{p+1}^{s_{1}}, \\
& \quad \vdots \\
& f_{m}=f_{m-p-1 l-p} x_{l} x_{p+1}^{s_{l-1}}+\cdots+f_{m-p-11} x_{p+1}^{s_{1}},
\end{aligned}
$$

where $1 \leqq s_{1} \leqq \cdots \leqq s_{l-p}$ are integers, $\int_{i j}$ are analytic functions in $\therefore, \cdots, x_{p+\jmath}$.

Here $f^{-1} f(0)=\left\{x_{1}=\cdots x_{p+1}=0\right\}$. Hence $d_{f}(0)=n-p-1$. Since $l \geqq p+1$ and $d_{f} \leqq n-l, l$ must be equal to $p+1$. Hence we have $d_{f}(0)=n-l$. which proves Proposition 9.1.

Proposition 9.2 (Complex case). If $f$ is piecewise linearizable, then $d_{f}=$ $2(n-l)$ globally.

Proof. Proposition 9.1 tells us that $d_{f} \leqq 2(n-l)$ and that $\dot{u}_{i}=2(n-l)$ on an open dense subset of $M_{1}$. As the problem is local, we assume $Y_{2}=C^{m}$, and we write $f=\left(f_{1}, \cdots, f_{m}\right)$. For any $x \in M_{1}$, let $\mathcal{O}_{x}$ be the ring of analytic function germs on $M_{1}$ at $x$, and $\mathfrak{p}_{x}$ be the ideal of $\mathcal{O}_{x}$ generated by $\dot{f}_{1}-\dot{f}_{1}(x), \cdots, f_{m}-$ $f_{m}(x)$. By Proposition 1, Chapter 1, [18], the function $M_{1} \equiv x \rightarrow$ coheight $\mathfrak{p}_{x}$ is upper semi-continuous. On the other hand, by Hilbert zero point theorem we have

$$
d_{f}(x)=2 \text { coheight } \mathfrak{p}_{. c}, x \in M_{1},
$$

Hence $d_{f}$ is an upper semi-continuous function on $M_{1}$. That prores Proposition 9.2.

Example 9.3. A simple example of map not piecewise linearizable is

$$
f:(x, y) \longrightarrow(x y, x) \text {. }
$$

\section{References}

1_ Gibson, C. G., Wirthmüller, K., du Plessis, A. A. and Looijenga, E. I. N.. Topological stability of smooth mappings, Lecture Notes in Math., 552 (1976). Springer.

$\therefore 2^{-}$Giesecke, B., Simpliziable Zerlegung abzählbarer analytische Ráume, Math. Zeit., 83 (1964), 177-213.

3.- Hironaka, H., Subanalytic sets, Number theory', algebraic geometry chd commutatin algebra, in honour of Y. Akizuki, 1973, 453-493, Kinokuniya, Tokyo.

[4] - Triangulations of algebraic sets, Algebraic geometry, Proc. Symp. Pure Math., AMS, 29 (1975), 165-185.

[5] Hirsch, M. W., Differential topology, GTM, 33 Springer, 1976.

[6] Kirby, R.C. and Siebenmann, L.C., Foundational essay's on topolugicol manifolds. smoothings, and triangulations, Ann. Math. Studies, 88. Princeton Univ. Press, 1977.

[7- Koopman, B.C. and Brown, A.B., On the covering of analytic loc. wy complexes, Trans. Amer. Soc., 34 (1932), 231-251.

:8 Lefschetz, S., Topology, Amer. Math. Soc. Coll. Publ., New York. 1930. 
[9] Lefschetz, S. and Whitehead, J.H.C., On analytical complexes, Trans. Amer. Math. Soc., 35 (1933). 510-517.

[10] Łojasiewicz, S., Triangulation of semi-analytic sets, Ann. Scu. Norm., Pisa, 18 (1964), 449-47t.

[11] Mather, J.N., Stratifications and mappings, Dynamical systems, M. M. Peixoto, Academic Press, (1973), 195-223.

[12] Munkres, J.R., Elementary differential topology, Ann, Math. Studies, 54, Princeton Univ. Press, 1963.

[13] Rourke, C.P. and Sanderson, B. J., Introduction to piecevvise-linear topology, Erg. d. Math., 69. Springer, 1976.

[14] Sato, K., Local triangulation of real analytic varieties, Osaka Math. J., 1j (1963), 109-125.

[15] Shiota, M., Transformations of germs of differentiable functions through changes of local coordinates, Publ. RIMS, Kyoto Univ., 9 (1973), 123-140.

[16] - Equivalence of differentiable mappings and analytic mappings, Publ. Math. IHES, 5џ (1981), 37-122.

[17] Classification of Nash Manifolds, Ann. Inst. Fourier, Grenoble, 33 (1983), 209-232.

[18] Tougeron, J. C.. Idéaux de fonctions différentiables I, Ann. Inst. Fourier, Grenoble, 18 (1968), $17 \mathfrak{\tau}-240$.

[19] van der Waerden, B.L., Topologische Begründung des Kalküls der abzählenden Geometrie, Math. Ann. 102 (1929), 337-362.

[20] Whitney, H., Analytic extensions of differentiable functions defined in closed sets, Trans. Amer. Math. Soc., 36 (1934), 63-89. 